\title{
A type $A$ structure in Khovanov homology
}

\author{
LAWRENCE P ROBERTS
}

\begin{abstract}
Inspired by bordered Floer homology, we describe a type $A$ structure in Khovanov homology, which complements the type $D$ structure previously defined by the author. The type $A$ structure is a differential module over a certain algebra. This can be paired with the type $D$ structure to recover the Khovanov chain complex. The homotopy type of the type $A$ structure is a tangle invariant, and homotopy equivalences of the type $A$ structure result in chain homotopy equivalences on the Khovanov chain complex found from a pairing. We use this to simplify computations and introduce a modular approach to the computation of Khovanov homologies. Several examples are included, showing in particular how this approach computes the correct torsion summands for the Khovanov homology of connect sums. A lengthy appendix is devoted to establishing the theory of these structures over a characteristic-zero ring.
\end{abstract}

57M27; 55N35

\section{Introduction}

In a previous paper, we described an algebra $\mathcal{B} \Gamma_{n}$ for a set of $2 n$ points $P_{2 n}$ ordered along a line (summarized in the next section) and a type $D$ structure $\llbracket \vec{T} \rrbracket$ for an outside tangle $\vec{T}$ whose endpoints are these $2 n$ points, where an outside tangle is one with a diagram in an oriented half-plane whose boundary contains $P_{2 n}$ but provides $P_{2 n}$ with the opposite linear ordering when inherited from the boundary orientation; see Roberts [9]. In this paper, we consider inside tangles: tangles where the orientation on the boundary equips $P_{2 n}$ with the same ordering. We will picture these as lying on the left side of the $y$-axis in $\mathbb{R}^{2}$. For example, the following is an inside tangle $\overleftarrow{T}$ over $P_{4}$ when the plane has its usual orientation:

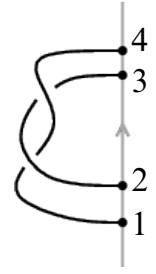

These tangles will be taken with an orientation, although we suppress that data for the introduction. To such a tangle we will associate a bigraded module $\langle\overleftarrow{T} \rrbracket$ and a differential $d_{\mathrm{APS}}$, which is a modified version of the differential defined by M Asaeda, J Przytycki, and A Sikora in [1], [2]. It is modified to have more generators, in a manner similar to Khovanov's invariant for tangles by [7]. 
From there we define a bigrading preserving right action $\left\langle\vec{T} \rrbracket \otimes \mathcal{B} \Gamma_{n} \rightarrow\langle\vec{T} \rrbracket\right.$ which is compatible with $d_{\mathrm{APS}}$ by a certain Leibniz identity. This will make $\langle\vec{T} \rrbracket$ into a differential right module over $\mathcal{B} \Gamma_{n}$. If we consider this within a suitable category of right $A_{\infty}$-modules we have a notion of homotopy equivalence of right modules. We will then show that Reidemeister moves on the diagram $\overleftarrow{T}$ will produce homotopy equivalent $A_{\infty}$-modules. We do this over $\mathbb{Z}$ with a somewhat different sign convention than usual, and a good bit of this paper is devoted to ensuring that the sign choices will work (the reader should consider that there are different sign conventions that can be followed in the Khovanov construction, and that these will produce distinct even and odd versions; we only consider the original, even, version here). Following the conventions of bordered Floer homology as used in Lipshitz, Ozsváth and Thurston [8], we will call this a type $A$ structure.

We arrange these constructions so that the following argument will work. Consider the following knot $K$ cleaved transversely in half by the $y$-axis:

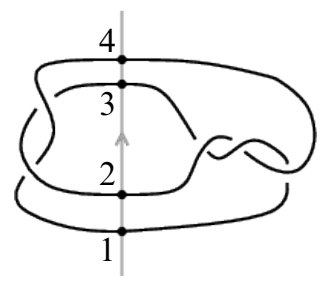

On the left side of the $y$-axis we recognize the inside tangle $\overleftarrow{T}$. On the right side, there is an outside tangle $\vec{T}$ :

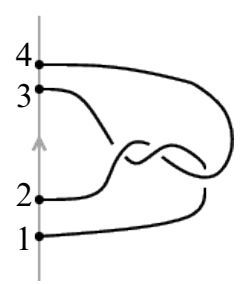

The Khovanov complex $\langle\langle K\rangle$ is generated by states consisting of a smoothing at each crossing of a diagram for $K$ and a decoration of $\{+,-\}$ attached to each planar circle resulting from the smoothing. Such a state $\xi$ might look like this:

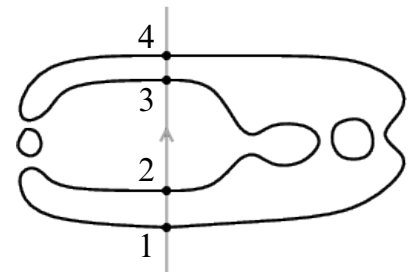


We can similarly divide this resolution along the $y$-axis; however, we do this in a less obvious way. The left side will be the diagram obtained by forgetting the circles on the right which do not intersect the $y$-axis. We similarly describe the right side:
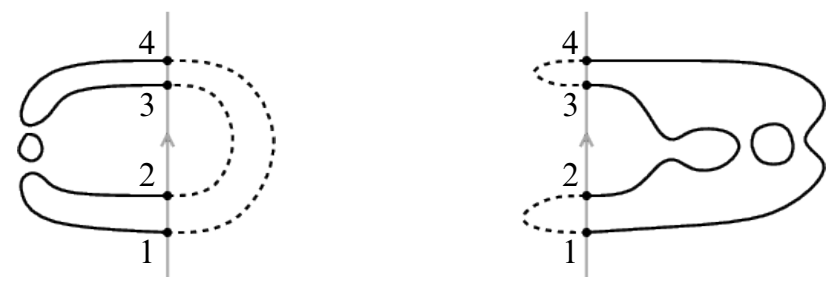

These are states, $\overleftarrow{\xi}$ and $\vec{\xi}$, generating summands in $\langle\overleftarrow{T} \rrbracket$ and $\llbracket \vec{T}\rangle$, respectively. To obtain the resolution of $K$ we will consider these to be glued along their common cleaved link:

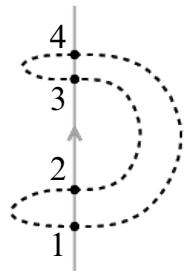

The latter diagram corresponds to an idempotent in $\mathcal{B} \Gamma_{n}$ which acts on the two states as the identity. These idempotents will be orthogonal in $\mathcal{B} \Gamma_{n}$, and if we let $\mathcal{I}$ be the idempotent subalgebra, then $\left\langle\overleftarrow{T} \rrbracket \otimes_{\mathcal{I}} \llbracket \vec{T}\right\rangle$ will be isomorphic to $\langle\langle K\rangle$, and $\overleftarrow{\xi} \otimes \vec{\xi}$ will represent $\xi$ in this decomposition. The Khovanov differential can then be decomposed into the contribution of the crossings on the right and left. However, these contributions can change the cleaved link, and the corresponding idempotent. We record the changed in the cleaved link with the algebra $\mathcal{B} \Gamma_{n}$. For the crossings on the right we obtain a map $\left.\vec{\delta}: \llbracket \vec{T}\rangle \rightarrow \mathcal{B} \Gamma_{n} \otimes_{\mathcal{I}} \llbracket \vec{T}\right\rangle$ which satisfies the requirements of a type $D$ structure described by Lipshitz, Ozsváth and Thurston [8]. The crossings on the left give rise to the type $A$ structure.

Following the constructions in [8] we can combine the type $A$ structure on $\langle\overleftarrow{T} \rrbracket$ and the type $D$ structure on $\llbracket \vec{T}\rangle$ into a chain complex $\langle\langle\overleftarrow{T} \rrbracket \otimes \llbracket \vec{T}\rangle$ with underlying module $\left\langle\overleftarrow{T} \rrbracket \otimes_{\mathcal{I}} \llbracket \vec{T}\right\rangle$ and differential

$$
\partial^{\bigotimes}(x \otimes y)=d_{\mathrm{APS}}(x) \otimes|y|+\left(m_{2} \otimes \mathbb{I}\right)(x \otimes \vec{\delta}(y)),
$$

where $m_{2}$ is the action on $\langle\overleftarrow{T} \rrbracket$. We then show that $\langle\langle T\rangle \cong\langle\overleftarrow{T} \rrbracket \otimes \llbracket \vec{T}\rangle$

Furthermore, changing either $\langle\overleftarrow{T} \rrbracket$ by a homotopy equivalence (of type $A$ structures) or $\llbracket \vec{T}\rangle$ (of type $D$ structures) changes $\langle\langle\overleftarrow{T} \rrbracket \otimes \llbracket \vec{T}\rangle$ by a chain homotopy equivalence. 
Thus, we can construct and simplify the two factors independently of each other, and then combine them using the $\nabla$-construction from [8].

This provides a fully modular approach to constructing Khovanov homology at the level of bigraded homotopy types. In particular we can simplify the structures for a tangle by homotopy equivalences before combining them with each other or any other tangle. For example, in Section 6 we will compute the type $A$ structures for tangles underlying the three Reidemeister moves, and simplify them, to see that they are homotopy equivalent to the type $A$ structure after applying the move. The pairing through $\nabla$ immediately implies the Reidemeister invariance of Khovanov homology. In short, we obtain a convenient means for understanding local modifications of link diagrams and their effects on the global Khovanov homology.

Ours is not the only approach to defining a Khovanov homology for tangles and their pairings. Khovanov defined a tangle homology in [7] for $(m, n)$-tangles which can be paired at the level of chain complexes to reflect the composition of tangles. However, our approach records the information needed for gluing differently, and this necessitates the use of the algebraic techniques of bordered Floer homology rather than those employed in [7].

Example Consider the following connected sum:

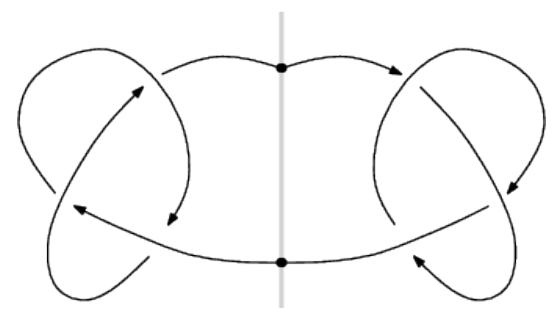

We showed in [9] that $\mathcal{B} \Gamma_{1}$ is the quiver algebra for this:

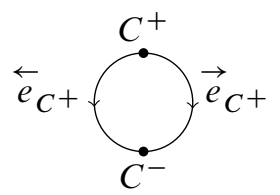

In [9] we showed that, for this example, $\llbracket \vec{T} \rrbracket$ is homotopy equivalent to

$$
\begin{aligned}
\vec{\delta}\left(s_{(-3,-15 / 2)}^{+}\right)=2 \overrightarrow{e_{C}} \otimes s_{(-2,-13 / 2)}^{-}+\overleftarrow{e_{C}} \otimes s_{(-3,-17 / 2)}^{-}, \\
\vec{\delta}\left(s_{(-2,-11 / 2)}^{+}\right)=-\overleftarrow{e_{C}} \otimes s_{(-2,-13 / 2)}^{-}, \quad \vec{\delta}\left(s_{(0,-3 / 2)}^{+}\right)=-\overleftarrow{e_{C}} \otimes s_{(0,-5 / 2)}^{-},
\end{aligned}
$$

where the + and - superscripts identify which idempotent acts as the identity on the 
generator. Furthermore, the type $A$ structure $\langle\overleftarrow{T} \rrbracket$ is homotopy equivalent to one which also has six generators:

$$
t_{(0,5 / 2)}^{+}, \quad t_{(2,13 / 2)}^{+}, \quad t_{(3,17 / 2)}^{+}, \quad t_{(0,3 / 2)}^{-}, \quad t_{(2,11 / 2)}^{-}, \quad t_{(3,15 / 2)}^{-} .
$$

For these generators $d_{\mathrm{APS}} \equiv 0$, the action of $\overrightarrow{e_{C}}$ is given by

$$
t_{(0,5 / 2)}^{+} \rightarrow t_{(0,3 / 2)}^{-}, \quad t_{(2,13 / 2)}^{+} \rightarrow t_{(2,11 / 2)}^{-}, \quad t_{(3,17 / 2)}^{+} \rightarrow t_{(3,15 / 2)}^{-},
$$

and the action of $\overleftarrow{e_{C}}$ is given by $t_{(2,13 / 2)}^{+} \rightarrow 2 \cdot t_{(3,15 / 2)}^{-}$. The complex $\langle\overleftarrow{T} \rrbracket \otimes \llbracket \vec{T}\rangle$ can be computed exactly (see Section 8 for the details). It has homology with free part

$$
\mathbb{Z}_{(-3,7)} \oplus \mathbb{Z}_{(-2,-3)} \oplus \mathbb{Z}_{(-1,-3)} \oplus \mathbb{Z}_{(0,-1)}^{2} \oplus \mathbb{Z}_{(0,1)}^{2} \oplus \mathbb{Z}_{(1,3)} \oplus \mathbb{Z}_{(2,3)} \oplus \mathbb{Z}_{(3,7)}
$$

and torsion part

$$
(\mathbb{Z} / 2)_{(-2,-5)} \oplus(\mathbb{Z} / 2 \mathbb{Z})_{(0,-1)} \oplus(\mathbb{Z} / 2 \mathbb{Z})_{(1,1)} \oplus(\mathbb{Z} / 2 \mathbb{Z})_{(3,5)},
$$

which is the Khovanov homology of this knot.

Outline of the paper We construct the algebras $\mathcal{B} \Gamma_{n}$ and their differentials in Section 2. This includes a fuller description of cleaved links. In Section 3 we follow with a description of the bigraded module $\langle T \rrbracket$ we will associate to an inside tangle diagram, and equip this module with a differential modified from that found in Asaeda, Przytycki, and Sikora [2]. We then define the action of $\mathcal{B} \Gamma_{n}$ on $\langle T \rrbracket$ in Section 4, and prove that the differential and action turn $\left\langle T \rrbracket\right.$ into a bigraded differential module over $\mathcal{B} \Gamma_{n}$. In Section 5 we recall the algebra of type $A$ structures ( $A_{\infty}$-modules) from [8] and how they can be simplified through homotopy equivalences. We put this knowledge to work in Section 6, where we show that the model tangles before and after a Reidemeister move have type $A$ structures that are homotopy equivalent as type $A$ structures and provide other example computations. In Section 7 we prove that we can pair the type $A$ structure for an inside tangle with the type $D$ structure for an outside tangle, using a pairing technique from [8], and recover the chain complex for Khovanov homology. The construction respects the homotopy equivalences of type $A$ and type $D$ structures, so we can simplify these structures using homotopy equivalences first, and then pair to get a chain complex homotopy equivalent to the Khovanov chain complex. Finally, in Section 8 we give examples of the pairing theorem in action, including the details of the previous example. The pairing theorem rest upon an edifice of algebra described in [8] for characteristic-two coefficients. In this paper we need to lift to integer coefficients in a manner respecting the Khovanov sign conventions. The Appendix details the choices of signs which are both compatible with Khovanov's choices and allow us to replicate the algebraic results of [8]. See the beginning of the Appendix for a more detailed summary. 
Degree shift convention If $M$ is a $\mathbb{Z}$-graded module, $M[n]$ is the graded module with $(M[n])_{i}=M_{i-n}$, ie the module found by shifting the homogeneous elements of $M$ up $n$ levels. If $m \in M$, the corresponding element in $M[n]$ will be denoted $m[n]$. Thus $\operatorname{gr}(m[n])=\operatorname{gr}(m)+n$.

Acknowledgements After posting this paper to the arXiv, the author was informed by Cotton Seed that he had independently discovered a similar construction of a type $A$ structure in Khovanov homology. The author would like to thank Andy Manion for finding an error in the original version of this paper, and the referee for many helpful comments. This research was supported by a research grant from Research Grants Committee of the University of Alabama, Tuscaloosa.

\section{The algebra from cleaved links}

We summarize the construction of the algebra $\mathcal{B} \Gamma_{n}$ from [9].

\subsection{Cleaved planar links}

Let $P_{n}$ be the set of points $p_{1}=(0,1), \ldots, p_{2 n}=(0,2 n)$ on the $y$-axis of $\mathbb{R}^{2}$, ordered by the second coordinate. We denote the closed half-plane $(-\infty, 0] \times \mathbb{R} \subset \mathbb{R}^{2}$ by $\overleftarrow{\mathbb{H}}$ while $\overrightarrow{\mathbb{H}}=[0, \infty) \times \mathbb{R}$

Definition 2.1 A $n$-cleaved link $L$ is an embedding of circles in $\mathbb{R}^{2}$ such that

(1) the circles of $L$ are disjoint and transverse to the $y$-axis,

(2) each point in $P_{n}$ is on a circle in $L$,

(3) each circle in $L$ contains at least two points in $P_{n}$.

The set of circle components of $L$ will be denoted $\operatorname{CIR}(L)$.

We take two $n$-cleaved links to be equivalent if they are related by an isotopy of $\mathbb{R}^{2}$ which pointwise fixes the $y$-axis. We will denote the equivalence classes by $\widehat{\mathcal{C L}}_{n}$.

Definition 2.2 The constituents of an $n$-cleaved link $L$ are the planar matchings

$$
\overleftarrow{L}=\overleftarrow{\mathbb{H}} \cap L \quad \text { and } \quad \vec{L}=\overleftrightarrow{\mathbb{H}} \cap L
$$

Definition 2.3 A bridge for a cleaved link $L$ is an embedding $\gamma:[0,1] \rightarrow \mathbb{R}^{2} \backslash(\{0\} \times \mathbb{R})$ such that

(1) $\gamma(0)$ and $\gamma(1)$ are on distinct $\operatorname{arcs}$ of $\overleftarrow{L}$ or $\vec{L}$

(2) the image under $\gamma$ of $(0,1)$ is disjoint from $L$. 
By definition, a bridge $\gamma$ has image in either $\overrightarrow{\mathbb{H}}$ or $\overleftarrow{\mathbb{H}}$. We call this half-plane the location of the bridge. Bridges are also considered up to isotopy fixing the $y$-axis.

Definition 2.4 The equivalence classes of bridges for a cleaved link $L$ will be denoted BRIDGE $(L)$. BRIDGE $(L)=\overleftarrow{\mathrm{BR}}(L) \cup \overrightarrow{\mathrm{BR}}(L)$, where $\overleftarrow{\mathrm{BR}}(L)$ consists of those equivalence classes in $\overleftarrow{\mathbb{H}}$ and $\overrightarrow{\mathrm{BR}}(L)$ consists of those classes in $\overrightarrow{\mathbb{H}}$.

For each class of bridges $\gamma \in \operatorname{BRIDGE}(L)$ we can construct a new cleaved planar link.

Definition 2.5 Let $L$ be an equivalence class of cleaved links and let $\gamma \in \operatorname{BRIDGE}(L)$. $L_{\gamma}$ is the equivalence class of cleaved links found by surgery along $\gamma$.

$L_{\gamma}$ has a special bridge $\gamma^{\dagger}$ introduced by the surgery. More specifically, there is a neighborhood of $\gamma$ homeomorphic to $[-1,1] \times[-1,1]$ which intersects $L$ along $\{ \pm 1\} \times[-1,1]$, and for which $\gamma$ is the core $[-1,1] \times\{0\} . L_{\gamma}$ results from removing these two arcs from $L$ and replacing them with $[-1,1] \times\{ \pm 1\}$. The bridge $\gamma^{\dagger}$ is then the bridge for $L_{\gamma}$ defined by the cocore $\{0\} \times[-1,1]$.

Definition 2.6 (1) The support of $\gamma \in \operatorname{BRIDGE}(L)$ is the set of three circles in $L$ and $L_{\gamma}$ which contain the feet of $\gamma$ and $\gamma^{\dagger}$.

(2) $\operatorname{MERGE}(L)$ is the subset of $\gamma \in \operatorname{BRIDGE}(L)$, where surgery on $\gamma$ merges two circles $\left\{C_{a}(\gamma), C_{b}(\gamma)\right\}$. In this case, $C_{\gamma}$ is the circle in $\operatorname{CIR}\left(L_{\gamma}\right)$ which contains both feet of $\gamma^{\dagger}$.

(3) $\operatorname{DIVIDE}(L)$ is the subset of $\gamma \in \operatorname{BRIDGE}(L)$, where surgery on $\gamma$ divides a circle $C$ of $L$. In this case, $C_{\gamma}^{a}$ and $C_{\gamma}^{b}$ are the circles in $\operatorname{CIR}\left(L_{\gamma}\right)$ which contain the feet of $\gamma^{\dagger}$.

Proposition 2.7 Given any bridge $\gamma$ for $L$, BRIDGE $(L) \backslash\{\gamma\}$ can be decomposed as a disjoint union $B_{\pitchfork}(L, \gamma) \cup B_{||}(L, \gamma)$, where

(1) $B_{\pitchfork}(T, \gamma)$ consists of the classes of bridges whose representatives all intersect $\gamma$,

(2) $B_{\|}(L, \gamma)$ consists of the classes of bridges containing a representative which does not intersect $\gamma$.

Furthermore, we can divide $B_{||}(L, \gamma)$ into the disjoint union $B_{d}(L, \gamma) \cup B_{s}(L, \gamma) \cup$ $B_{o}(L, \gamma)$, where

(1) $B_{d}(L, \gamma)$ consists of those bridges neither of whose ends is on an arc with $\gamma$,

(2) $B_{S}(L, \gamma)$ consists of those bridges with a single end on the same arc as $\gamma$ and lying on the same side of the arc as $\gamma$,

(3) $B_{o}(L, \gamma)$ consists of those bridges with a single end on the same arc as $\gamma$ and lying on the opposite side of the arc as $\gamma$. 
If $\eta$ is in $B_{*}(L, \gamma)$, where $*$ represents a specific choice of one of the above, then $\gamma \in B_{*}(L, \eta)$. Furthermore, if $\delta$ has a different location than $\gamma$ then $\delta \in B_{d}(L, \gamma)$. We consider how these sets change under surgery on $\gamma$.

Proposition 2.8 Surgery on $\gamma$ induces an identification $B_{d}(L, \gamma)$ with $B_{d}\left(L_{\gamma}, \gamma^{\dagger}\right)$ and a $2: 1$ map $B_{s}(L, \gamma) \rightarrow B_{o}\left(L_{\gamma}, \gamma^{\dagger}\right)$. Dually, there is a $2: 1$ map $B_{s}\left(L_{\gamma}, \gamma^{\dagger}\right) \rightarrow$ $B_{o}(L, \gamma)$.

Proof Let $\eta \in B_{\|}(L, \gamma)$. Pick a representative arc for $\eta$ which does not intersect the representative arc for $\gamma$. Then $\eta$ also represents a bridge in $B_{||}\left(L_{\gamma}, \gamma^{\dagger}\right)$, and vice versa. If $\eta \in B_{d}(L, \gamma)$, any isotopy of the representative arc occurs in a region disjoint from $\gamma$ and its endpoints, since the isotopy will occur along arcs disjoint from those intersecting $\gamma$. This isotopy also survives into $\left(L_{\gamma}, \gamma^{\dagger}\right)$. Reversing this construction for $B_{d}\left(L_{\gamma}, \gamma^{\dagger}\right)$ proves the identification. Note that an isotopy of $\eta \in B_{s}(L, \gamma)$ missing $\gamma$ can likewise be pushed forward. However, for each $\eta$ we can slide $\eta$ over $\gamma$ to get another bridge $\eta^{\prime} \in B_{s}(L, \gamma)$. In $L_{\gamma}, \eta \simeq \eta^{\prime}$, and both are on the opposite side of $\gamma^{\dagger}$. By looking at a local model, this is the only type of collision, so the map is $2: 1$ on $B_{S}(L, \gamma)$. We can apply the same argument to $\left(L_{\gamma}, \gamma^{\dagger}\right)$ to obtain the $2: 1$ map in the other direction.

A decoration for an $n$-cleaved link $L$ is a map $\sigma: \operatorname{CIR}(L) \rightarrow\{+,-\}$.

Definition 2.9 $\mathcal{C} \mathcal{L}_{n}$ is the set of decorated, $n$-cleaved links:

$$
\mathcal{C} \mathcal{L}_{n}=\left\{(L, \sigma) \mid L \in \widehat{\mathcal{C L}}_{n}, \sigma \text { is a decoration for } L\right\} .
$$

We will often restrict a decoration $\sigma$ of $L$ to give decorations on the arcs of its constituents $\overleftarrow{L}$ and $\vec{L}$. In addition, we will need to following statistic for a decorated, cleaved link:

$$
\iota(L, \sigma)=\#\{C \in \operatorname{CIR}(L) \mid \sigma(C)=+\}-\#\{C \in \operatorname{CIR}(L) \mid \sigma(C)=-\} .
$$

\subsection{The algebra $\mathcal{B} \Gamma_{n}$}

We will describe $\mathcal{B} \Gamma_{n}$ by generators and relations. First, there is an idempotent for each decorated, cleaved link in $\mathcal{C} \mathcal{L}_{n}$. We will denote the idempotent corresponding to $(L, \sigma)$ by $I_{(L, \sigma)}$. The idempotents will be orthogonal to each other.

Definition 2.10 $\mathcal{I}_{n}$ is the sub-algebra generated by the idempotents $I_{(L, \sigma)}$. 
For each $(L, \sigma) \in \mathcal{C} \mathcal{L}_{n}$ we specify certain elements in $I_{(L, \sigma)} \mathcal{B} \Gamma_{n} . \mathcal{B} \Gamma_{n}$ will be freely generated by the idempotents and these elements, subject to the relations described below:

(1) For each circle $C \in \operatorname{CIR}(L)$ with $\sigma(C)=+$ there are two elements $\overrightarrow{e_{C}}$ and $\overleftarrow{e_{C}}$ in $I_{(L, \sigma)} \mathcal{B} \Gamma_{n}$. Furthermore, $\vec{e}_{C} I_{\left(L, s_{C}\right)}=\overrightarrow{e_{C}}$ for the decoration with $s_{C}(C)=-$ and $s_{C}(D)=s(D)$ for each $D \in \operatorname{CIR}(L) \backslash\{C\}$, while $\overrightarrow{e_{C}} I_{\left(L^{\prime}, s^{\prime}\right)}=0$ for every other idempotent. The same relations hold for $\overleftarrow{e_{C}}$. These types of elements are called decoration elements, while the $C$ above is called the support of the element.

(2) Let $\gamma \in \operatorname{BRIDGE}(L)$. There is a bridge element $e_{\left(\gamma ; \sigma, \sigma_{\gamma}\right)}$ with $I_{(L, \sigma)} e_{\left(\gamma ; \sigma, \sigma_{\gamma}\right)}=$ $e_{\left(\gamma ; \sigma, \sigma_{\gamma}\right)} I_{\left(L_{\gamma}, \sigma_{\gamma}\right)}=e_{\left(\gamma ; \sigma, \sigma_{\gamma}\right)}$ in each of the following cases, based on the decorations:

(a) $\gamma \in \operatorname{MERGE}(L), \sigma$ and $\sigma_{\gamma}$ restrict to the support of $\gamma$ as one of the following three configurations:

$$
\begin{array}{lll}
\sigma\left(C_{a}(\gamma)\right)=+, & \sigma\left(C_{b}(\gamma)\right)=+, & \sigma_{\gamma}\left(C_{\gamma}\right)=+; \\
\sigma\left(C_{a}(\gamma)\right)=-, & \sigma\left(C_{b}(\gamma)\right)=+, & \sigma_{\gamma}\left(C_{\gamma}\right)=-; \\
\sigma\left(C_{a}(\gamma)\right)=+, & \sigma\left(C_{b}(\gamma)\right)=-, & \sigma_{\gamma}\left(C_{\gamma}\right)=-;
\end{array}
$$

and $s(D)=s_{\gamma}(D)$ on every circle not in the support of $\gamma$.

(b) $\gamma \in \operatorname{DIVIDE}(L), C \in \operatorname{CIR}(L)$ is the circle containing both feet of $\gamma$, one of the following two conditions holds:

(i) $\sigma(C)=+, \sigma_{\gamma}$ restricts to the support of $\gamma$ as either of the configurations

$$
\begin{array}{cc}
\sigma_{\gamma}\left(C_{\gamma}^{a}\right)=+, & \sigma_{\gamma}\left(C_{\gamma}^{b}\right)=-; \\
\sigma_{\gamma}\left(C_{\gamma}^{a}\right)=-, \quad \sigma_{\gamma}\left(C_{\gamma}^{b}\right)=+;
\end{array}
$$

(ii) $\sigma(C)=-, \sigma_{\gamma}$ restricts to the support of $\gamma$ as

$$
\sigma_{\gamma}^{-}\left(C_{\gamma}^{a}\right)=-, \quad \sigma_{\gamma}^{-}\left(C_{\gamma}^{b}\right)=-;
$$

and $s(D)=s_{\gamma}(D)$ on every circle not in the support of $\gamma$.

In [9], we note that with these generators and idempotents, $\mathcal{B} \Gamma_{n}$ is finite-dimensional.

Proposition 2.11 $\mathcal{B} \Gamma_{n}$ is finite-dimensional.

Furthermore, $\mathcal{B} \Gamma_{n}$ can be given a bigrading [9]. On the generating elements the bigrading is specified by setting

$$
I_{(L, \sigma)} \rightarrow(0,0), \quad \overrightarrow{e_{C}} \rightarrow(0,-1), \quad \overleftarrow{e_{C}} \rightarrow(1,1), \quad \overrightarrow{e_{\gamma}} \rightarrow\left(0,-\frac{1}{2}\right), \quad \overleftarrow{e_{\gamma}} \rightarrow\left(1, \frac{1}{2}\right)
$$


On every other element it is computed by extending the above homomorphically. The first entry of this bigrading will be denoted $\overleftarrow{l}(\alpha)$, while the second element will be denoted $q(\alpha)$.

We now turn to describing the relations between these generators. Each of the relations is homogeneous with respect to the bigrading. First, there are a number of graded commutativity relations, based on the first entry in the bigrading:

$$
e_{\alpha} e_{\beta^{\prime}}=(-1)^{\overleftarrow{l}\left(e_{\alpha}\right) \overleftarrow{l}\left(e_{\beta}\right)} e_{\beta} e_{\alpha^{\prime}}
$$

This graded commutativity occurs in the following cases, assuming that $I_{(L, \sigma)} e_{\alpha} \neq 0$ and $I_{(L, \sigma)} e_{\beta} \neq 0$ :

(1) $e_{\alpha}$ and $e_{\beta}$ are decoration elements for distinct circles $C$ and $D$ in $(L, \sigma)$ with $\sigma(C)=\sigma(D)=+$, and $e_{\alpha^{\prime}}$ is the decoration element for $D$ in $\left(L, \sigma_{C}\right)$, while $e_{\beta^{\prime}}$ is the decoration element for $C$ in $\left(L, \sigma_{D}\right)$.

(2) $e_{\alpha}=e_{\left(\gamma, \sigma, \sigma^{\prime}\right)}$ for a bridge $\gamma$ in $(L, \sigma)$ and $e_{\beta}$ is a decoration element for $C \in \operatorname{CIR}(L)$, with $C$ not in the support of $\gamma$, while $e_{\alpha^{\prime}}=e_{\left(\gamma, \sigma_{C}, \sigma_{C}^{\prime}\right)}$ and $e_{\beta^{\prime}}$ is the decoration element for $C$ in $\left(L_{\gamma}, s^{\prime}\right)$. Due to the disjoint support, there will always be a pair of such elements.

(3) $e_{\alpha}=e_{\left(\gamma, \sigma, \sigma^{\prime}\right)}$ and $e_{\beta}=e_{\left(\eta, \sigma, \sigma^{\prime \prime}\right)}$ are bridge elements for distinct bridges $\gamma$ and $\eta$ in $(L, \sigma)$, with $\eta \in B_{d}(L, \gamma), e_{\beta^{\prime}}=e_{\left(\eta, \sigma^{\prime}, \sigma^{\prime \prime \prime}\right)}$ and $e_{\alpha^{\prime}}=e_{\left(\gamma, \sigma^{\prime \prime}, \sigma^{\prime \prime \prime}\right)}$ for some decoration $\sigma^{\prime \prime \prime}$ on $L_{\gamma, \eta}$.

(4) $e_{\alpha}=e_{\left(\vec{\gamma}, \sigma, \sigma^{\prime}\right)}$ and $e_{\beta}=e_{\left(\vec{\eta}, \sigma, \sigma^{\prime \prime}\right)}$ are bridge elements for distinct right bridges $\gamma$ and $\eta$ in $(L, \sigma)$, and $e_{\beta^{\prime}}=e_{\left(\vec{\delta}, \sigma^{\prime}, \sigma^{\prime \prime \prime}\right)}$ and $e_{\alpha^{\prime}}=e_{\left(\vec{\omega}, \sigma^{\prime \prime}, \sigma^{\prime \prime \prime}\right)}$, such that $L_{\gamma, \delta}=$ $L_{\eta, \omega}$, and some compatible decoration $\sigma^{\prime \prime \prime}$.

(5) $e_{\alpha}=e_{\left(\overleftarrow{\gamma}, \sigma, \sigma^{\prime}\right)}$ and $e_{\beta}=e_{\left(\overleftarrow{\eta}, \sigma, \sigma^{\prime \prime}\right)}$ are bridge elements for distinct left bridges in $(L, \sigma)$, with $\overleftarrow{\eta} \in B_{o}(L, \overleftarrow{\gamma})$, and $e_{\beta^{\prime}}=e_{\left(\overleftarrow{\delta}, \sigma^{\prime}, \sigma^{\prime \prime \prime}\right)}, e_{\alpha^{\prime}}=e_{\left(\overleftarrow{\omega}, \sigma^{\prime \prime}, \sigma^{\prime \prime \prime}\right)}$ with $L_{\gamma, \delta}=L_{\eta, \omega}$, and some compatible decoration $\sigma^{\prime \prime \prime}$.

We also note that the type (decoration vs bridge) and the location are the same for $e_{\alpha}$ and $e_{\alpha^{\prime}}$ as well as for the pair $e_{\beta}$ and $e_{\beta^{\prime}}$. In fact, in all these relations elements of the algebra from $\overrightarrow{\mathbb{H}}$ will act like even elements for the $\mathbb{Z} / 2 \mathbb{Z}$-grading from $\overleftarrow{l}(\alpha)$, while elements from $\overleftarrow{\mathbb{H}}$ act like odd elements.

Other bridge relations Suppose $\gamma \in \overleftarrow{\mathrm{BR}}(L)$ and $\eta \in B_{\pitchfork}\left(L_{\gamma}, \gamma^{\dagger}\right)$. Then

$$
e_{\left(\gamma, \sigma, \sigma^{\prime}\right)} e_{\left(\eta, \sigma^{\prime}, \sigma^{\prime \prime}\right)}=0
$$

whenever $\sigma^{\prime}$ and $\sigma^{\prime \prime}$ are compatible decorations. 
Furthermore, suppose that there is a circle $C \in \operatorname{CIR}(L)$ with $\sigma(C)=+$, and there are elements $\vec{e}_{\left(\gamma, \sigma, \sigma^{\prime}\right)}$ and $\vec{e}_{\left(\gamma^{\dagger}, \sigma^{\prime}, \sigma_{C}\right)}$ for a bridge $\gamma \in \overrightarrow{\mathrm{BR}}(L)$. Then

$$
\vec{e}_{\left(\gamma, \sigma, \sigma^{\prime}\right)} \vec{e}_{\left(\gamma^{\dagger}, \sigma^{\prime}, \sigma_{C}\right)}=\vec{e}_{C} .
$$

Such a circle $C$ is unique for the choice of $\gamma$ and $\sigma^{\prime}$ and is called the active circle for $\gamma$.

Finally, suppose that $\overleftarrow{\alpha} \in B_{S}(L, \overleftarrow{\gamma})$. Let $\overleftarrow{\beta}$ be the bridge obtained by sliding $\overleftarrow{\alpha}$ over $\overleftarrow{\gamma}$ $\overleftarrow{\delta}$ be the image of $\overleftarrow{\alpha}$ and $\overleftarrow{\beta}$ in $L \overleftarrow{\gamma}, \overleftarrow{\zeta}$ be the image of $\overleftarrow{\alpha}$ and $\overleftarrow{\gamma}$ in $L \overleftarrow{\beta}$ and $\overleftarrow{\eta}$ be the image of $\overleftarrow{\beta}$ and $\overleftarrow{\gamma}$ in $L \overleftarrow{\alpha}$. Then

$$
\overleftarrow{e_{\alpha}} \overleftarrow{e_{\eta}}+\overleftarrow{e_{\beta}} \overleftarrow{e_{\zeta}}+\overleftarrow{e_{\gamma}} \overleftarrow{e_{\delta}}=0
$$

whenever there are compatible decorations on $L_{\alpha}, L_{\beta}, L_{\gamma}$, and $L_{\alpha, \eta}=L_{\beta, \zeta}=L_{\gamma, \delta}$, for the paired edges to exist.

Relations for decoration edges When the support of $e_{C}$ is not disjoint from that of $\vec{e}_{\left(\gamma, \sigma, \sigma_{\gamma}\right)}$ the relations are different depending upon the location of $e_{C}$.

The relations for $\overrightarrow{\boldsymbol{e}_{\boldsymbol{C}}}$ Suppose that $\gamma \in \operatorname{MERGE}(L)$ merges $C_{1}$ and $C_{2}$ to get $C \in$ $\operatorname{CIR}\left(L_{\gamma}\right)$, and $\sigma\left(C_{1}\right)=\sigma\left(C_{2}\right)=+$. Then

$$
\vec{e}_{C_{1}} m_{\left(\gamma, \sigma_{C_{1}}, \sigma_{C}\right)}=\vec{e}_{C_{2}} m_{\left(\gamma, \sigma_{C_{2}}, \sigma_{C}\right)}=m_{\left(\gamma, \sigma, \sigma_{\gamma}\right)} \overrightarrow{e_{C}} \text {. }
$$

Note that if $\sigma\left(C_{i}\right)=-$ for either $i=1$ or 2 , then there is no relation imposed.

Dually, if surgery on $\gamma \in \operatorname{DIVIDE}(L)$ divides circle $C \in \operatorname{CIR}(L)$ into $C_{1}$ and $C_{2}$ in $\operatorname{CIR}\left(L_{\gamma}\right)$, and $\sigma$ assigns + to $C$, then

$$
\overrightarrow{e_{C}} f_{\left(\gamma, \sigma_{C}, \sigma_{C, \gamma}\right)}=f_{\left(\gamma, \sigma, \sigma_{\gamma}^{1}\right)} \vec{e}_{C_{1}}=f_{\left(\gamma, \sigma, \sigma_{\gamma}^{2}\right)} \vec{e}_{C_{2}},
$$

where $\sigma_{\gamma}^{i}$ assigns + to $C_{i}$ and - to $C_{3-i}$.

The relations for $\overleftarrow{e_{C}}$ Suppose that $\gamma \in \operatorname{MERGE}(L)$ merges $C_{1}$ and $C_{2}$ to get $C \in$ $\operatorname{CIR}\left(L_{\gamma}\right)$, and $\sigma\left(C_{1}\right)=\sigma\left(C_{2}\right)=+$. Then

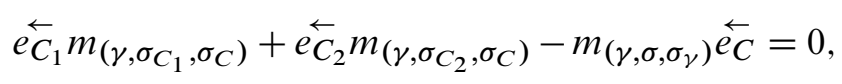

and when $\sigma(C)=+$ and $\gamma \in \operatorname{DIVIDE}(L)$ divides $C$ into $C_{1}$ and $C_{2}$,

$$
\overleftarrow{e_{C}} f_{\left(\gamma, \sigma_{C}, \sigma_{C, \gamma}\right)}+f_{\left(\gamma, \sigma, \sigma_{\gamma}^{1}\right)} e_{C_{1}}^{\overleftarrow{1}}-f_{\left(\gamma, \sigma, \sigma_{\gamma}^{2}\right)} e_{C_{2}}^{\overleftarrow{c}}=0
$$

whereas if $\gamma \in \operatorname{MERGE}(L)$ merges $C_{1}$ and $C_{2}$ to get $C \in \operatorname{CIR}\left(L_{\gamma}\right)$, and $\sigma\left(C_{1}\right)=$ $\sigma\left(C_{2}\right)=+$, then

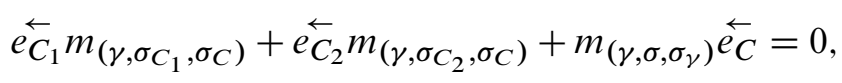


and when $\sigma(C)=+$ and $\gamma \in \operatorname{DIVIDE}(L)$ divides $C$ into $C_{1}$ and $C_{2}$

$$
{\overleftarrow{e_{C}}}_{\left(\gamma, \sigma_{C}, \sigma_{C, \gamma}\right)}+f_{\left(\gamma, \sigma, \sigma_{\gamma}^{1}\right)} e_{C_{1}}^{\leftarrow}+f_{\left(\gamma, \sigma, \sigma_{\gamma}^{2}\right)} e_{C_{2}}^{\leftarrow}=0
$$

\subsection{A differential on $\mathcal{B} \Gamma_{n}$}

Surgery along a bridge $\gamma \in \overleftarrow{\mathrm{BR}}(L)$ followed by surgery on $\gamma^{\dagger}$ does not correspond to a relation (compare relation (6)). Instead these products occur in a differential on $\mathcal{B} \Gamma_{n}$.

Proposition 2.12 [9] Let $(L, \sigma) \in \mathcal{C L}_{n}$ such that there is a circle $C \in \mathrm{CIR}(L)$ with $\sigma(C)=+$. Let $\overleftarrow{e_{C}}$ be the decoration element corresponding to $C$. Let

$$
d_{\Gamma_{n}}\left(\overleftarrow{e_{C}}\right)=-\sum e_{\left(\gamma, \sigma, \sigma_{\gamma}\right)} e_{\left(\gamma^{\dagger}, \sigma_{\gamma}, \sigma_{C}\right)}
$$

where the sum is over all $\gamma \in \overleftarrow{\mathrm{BR}}(L)$ with $C$ as active circle, and all decorations $\sigma_{\gamma}$ which define compatible elements. Let $d_{\Gamma_{n}}(e)=0$ for every other generator $e$ (including idempotents). Then $d_{\Gamma_{n}}$ can be extended to a $(1,0)$-differential on bigraded algebra $\mathcal{B} \Gamma_{n}$ which satisfies the following Leibniz identity:

$$
d_{\Gamma_{n}}(\alpha \beta)=(-1)^{\overleftarrow{l}(\beta)}\left(d_{\Gamma_{n}}(\alpha)\right) \beta+\alpha\left(d_{\Gamma_{n}}(\beta)\right)
$$

We denote this differential bigraded $\mathbb{Z}$-algebra by $\left(\mathcal{B} \Gamma_{n}, d_{\Gamma_{n}}\right)$.

Example Consider $\left(\mathcal{B} \Gamma_{1}, d_{\Gamma_{1}}\right) . P_{1}$ consists of two points, and there is only one planar matching in $\overleftarrow{\mathbb{H}}$ and $\overleftrightarrow{\mathbb{H}}$. Consequently, the only 1 -cleaved link is a circle intersecting the $y$-axis in two points. Thus, there are two vertices in $\Gamma_{1}$ : when this circle is decorated with $\mathrm{a}+$ and when it is decorated with a - . We will call these $C^{ \pm}$. There are no bridges in either $\overleftarrow{H}$ or $\overrightarrow{\mathbb{H}}$, so the only edges are $\overleftarrow{e}_{C}: C^{+} \rightarrow C^{-}$and $\vec{e}_{C}: C^{+} \rightarrow C^{-}$. Thus $\Gamma_{1}$ looks like this:

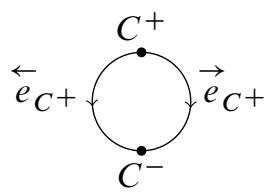

Thus, $\mathcal{B} \Gamma_{1}$ consists of four elements: $I_{C^{+}}, I_{C^{-}}$in grading $(0,0), \overleftarrow{e}_{C}$ in grading $(1,1)$, and $\vec{e}_{C}$ in grading $\left(0,-\frac{1}{2}\right)$. The product of any two of these is trivial except for the actions of the idempotents: $I_{C}+\overleftarrow{e}_{C}=\overleftarrow{e}_{C}=\overleftarrow{e}_{C} I_{C}$, and similarly for $\vec{e}_{C}$. The differential $d_{\Gamma_{1}} \equiv 0$ since its image is in the set generated by paths of bridge edges.

For more detail about $\left(\mathcal{B} \Gamma_{2}, d_{\Gamma_{2}}\right)$ see the examples in [9, Section 2]. 


\section{Tangles and resolutions}

In this section we recall the notions of tangles and resolutions used in [9], adapting them to the case at hand. More details are provided there.

Let $\overleftarrow{\mathbb{R}^{3}}=\overleftarrow{\mathbb{H}} \times \mathbb{R}$ be the half space corresponding to $\overleftarrow{\mathbb{H}} \subset \mathbb{R}^{2}$ under the standard projection $\pi$ to the $x y$-plane.

Definition 3.1 An (inside) tangle $\overleftarrow{\mathcal{T}}$ is a smooth, proper embedding of

(i) $n$ copies of the interval $[0,1]$, and

(ii) $k$ copies of $S^{1}$

in $\overleftarrow{\mathbb{R}}^{3}$, whose boundary is the set of $2 n$ points $P_{n}$ in $\partial \overleftarrow{\mathbb{H}}$. $\overleftarrow{\mathcal{T}_{1}}$ and $\overleftarrow{\mathcal{T}_{2}}$ are equivalent if there is an isotopy of $\mathbb{R}^{3}$ taking $\overleftarrow{\mathcal{T}}_{1}$ to $\overleftarrow{\mathcal{T}}_{2}$ and pointwise fixing the boundary $\partial \mathbb{R}^{3}$.

As usual, we will study $\overleftarrow{\mathcal{T}}$ through its tangle diagrams in $\overleftarrow{\mathbb{H}}$. Different diagrams for $\overrightarrow{\mathcal{T}}$ are related by sequences of Reidemeister moves, and planar isotopies, in the interior of $\overleftarrow{H}$. We will denote a tangle diagram for a tangle by the corresponding roman letter: $\overleftarrow{T}$ will be a diagram for $\overleftarrow{\mathcal{T}}$

The crossings of $\overleftarrow{T}$ form a set $\mathrm{CR}(\overleftarrow{T})$. We will orient $\overleftarrow{\mathcal{T}}$ and use the usual convention for positive and negative crossings:
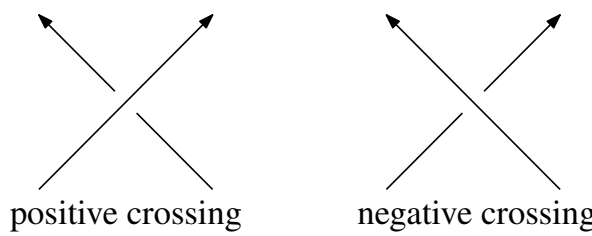

The number of positive/negative crossings will be denoted $n_{ \pm}(\overleftarrow{T})$

\subsection{Resolutions}

Definition 3.2 A resolution $r$ of $\overleftarrow{T}$ is a pair $(\rho, \overleftarrow{m})$, where $\rho: \operatorname{CR}(\overleftarrow{T}) \rightarrow\{0,1\}$ and $\vec{m}$ is a planar matching of $P_{2 n}$ embedded in $\overrightarrow{\mathbb{H}}$. The resolution diagram, $r(\overleftarrow{T})$ is the crossingless, planar link in $\overleftarrow{H}$ obtained by gluing $\overleftarrow{T} \subset \overleftarrow{H}$ to $\vec{m} \subset \overrightarrow{\mathbb{H}}$, and locally replacing (disjoint) neighborhoods of each crossing $c \in \mathrm{CR}(\overleftarrow{T})$ using the following rule:
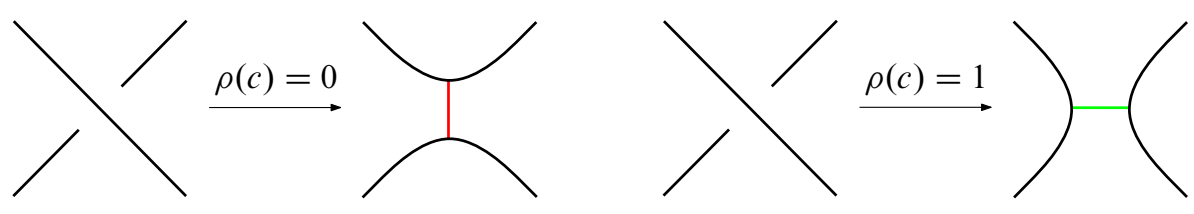

The set of resolutions will be denoted $\operatorname{RES}(\overleftarrow{T})$. 
The local arcs introduced by $\overleftarrow{T} \leftarrow \rho(\overleftarrow{T})$ are called resolution bridges. The resolution bridge for a crossing $c \in \mathrm{CR}(\overleftarrow{T})$ will be denoted $\gamma_{r, c}$ (or just $\gamma_{c}$ when the resolution is understood). If $\rho(c)=0$ we will call $\gamma_{c}$ an active bridge for $r$, while if $\rho(c)=1$ it will be called inactive. The active bridges for $r$ are the elements of the set $\operatorname{ACTIVE}(r)$. We will denote by $\gamma_{c}(r)$ the resolution obtained by surgering the diagram for $r$ along $\gamma_{c}$. The resolution bridges at $c$ for $\gamma_{c}(r)$ will be denoted by $\gamma_{c}^{\dagger}$ when considered from $r$. A resolution diagram $r(\overleftarrow{T})$ consists of a planar diagram of circles, which we divide into two groups: (1) the free circles which are contained in int $\overleftarrow{H}$ and are the elements of a set $\operatorname{FREE}(r)$, and (2) the cleaved circles which cross the $y$-axis, and which determine an element $\operatorname{cl}(r) \in \widehat{\mathcal{C L}}_{n}$.

Definition 3.3 A state for $\overleftarrow{T}$ is a pair $(r, s)$, where

(1) $r$ is a resolution of $\overleftarrow{T}$

(2) $s$ is an assignment of an element of $\{+,-\}$ to each circle of $r(\overleftarrow{T})$. This assignment will be called a decoration on $r(\overleftarrow{T})$

The states for $\overleftarrow{T}$ will be denoted $\operatorname{STATE}(\overleftarrow{T})$

Definition 3.4 The boundary of a state $(r, s)$ for $\overleftarrow{T}$ is the element $\partial(r, s)=(\operatorname{cl}(r), \sigma) \in$ $\mathcal{C} \mathcal{L}_{n}$, where $\sigma=\left.s\right|_{\mathrm{cl}(r)}$.

\subsection{A bigraded module spanned by the states}

Definition 3.5 For a state $(r, s) \in \operatorname{STATE}(\overleftarrow{T})$ with $r=(\rho, \vec{m})$, let

(1) $h(r)=\sum_{c \in \mathrm{CR}(\overleftarrow{T})} \rho(c)$

(2) $q(r, s)=\sum_{C \in \operatorname{FREE}(r)} s(C)$,

(3) $\iota(r, s)=\iota(L, \sigma)$, where $(L, \sigma)=\partial(r, s)$.

Let

$$
\overleftarrow{C K}(\overleftarrow{T}, L, \sigma) \cong \bigoplus_{\partial(r, s)=(L, \sigma)} \mathbb{Z} \cdot(r, s)
$$

where $(r, s)$ occurs in bigrading $\left(h(r)-n_{-}, h(r)+q(r, s)+\frac{1}{2} \iota(r, s)+n_{+}-2 n_{-}\right)$. The first entry will be called the homological grading of the state, while the second is its quantum grading.

Definition 3.6 The type $A$ module for an inside tangle $\overleftarrow{T}$ is

$$
\left\langle\overleftarrow{T} \rrbracket=\bigoplus_{(L, \sigma) \in \mathcal{C} \mathcal{L}_{n}} \overleftarrow{C K}(\overleftarrow{T}, L, \sigma)\right.
$$


There is a right action of the idempotent algebra $\mathcal{I}_{n} \subset \mathcal{B} \Gamma_{n}$ on $\langle\overleftarrow{T} \rrbracket$ :

$$
(r, s) \cdot I_{(L, \sigma)}= \begin{cases}(r, s) & \text { if } \partial(r, s)=(L, \sigma), \\ 0 & \text { otherwise. }\end{cases}
$$

Thus $I_{(L, \sigma)}$ acts non-trivially only on the summand $\overleftarrow{C K}(\overleftarrow{T}, L, \sigma)$

In addition the construction of Asaeda, Przytycki, and Sikora [1], in [2] $《 \overleftarrow{T} \rrbracket$ is endowed with a $(1,0)$-differential, $d_{\mathrm{APS}}$, described presently. With this differential, $\langle\overleftarrow{T} \rrbracket$ becomes a chain complex, and the main result of [2] implies that the (bigraded) homology of $\langle\overleftarrow{T} \rrbracket$ is an isotopy invariant of $\overleftarrow{\mathcal{T}}$, up to (bigraded) isomorphism.

\subsection{The differential from [2]}

To define the differential we first order the crossings of $\overleftarrow{T}$. Then for $(r, s) \in \operatorname{STATE}(\overleftarrow{T})$ we define

$$
d_{\mathrm{APS}}(r, s)=\sum_{\gamma \in \operatorname{ACTIVE}(r)}(-1)^{I(\rho, \gamma)} D_{\gamma, \rho}(r, s),
$$

where $r=(\rho, \vec{m}), I(\rho, \gamma)=\sum_{c_{\gamma}<c^{\prime}} \rho\left(c^{\prime}\right)$ is the number of $\rho$-inactive crossings which occur after the crossing $c$ corresponding to $\gamma$, and $D_{\gamma, \rho}$ is a map defined at each active arc. This map is prescribed by the following recipe:

(1) Khovanov case (i) Suppose surgery on $\gamma$ merges the free circles $C_{1}$ and $C_{2}$ in $\rho$ to get a free circle $C$ in $\gamma(\rho)$.

(a) If $s\left(C_{1}\right)=s\left(C_{2}\right)=+$, then $D_{\gamma, \rho}(r, s)=\left(\gamma(r), s^{\prime}\right)$, where $s^{\prime}(C)=+$ and $s^{\prime}(D)=s(D)$ for every circle $D \neq C_{1}, C_{2}, C$, free or cleaved.

(b) If either $s\left(C_{1}\right)=-$ and $s\left(C_{2}\right)=+$ or $s\left(C_{1}\right)=-$ and $s\left(C_{2}\right)=+$, then $D_{\gamma, \rho}(r, s)=\left(\gamma(r), s^{\prime \prime}\right)$, where $s^{\prime \prime}(C)=-$ and $s^{\prime \prime}(D)=s(D)$ for every circle $D \neq C_{1}, C_{2}, C$, free or cleaved.

(c) If $s\left(C_{1}\right)=s\left(C_{2}\right)=-$, then $D_{\gamma, \rho}(r, s)=0$.

(2) Khovanov case (ii) Suppose $\gamma$ has both feet on the same free circle $C$ in $r$, so that surgering $C$ along $\gamma$ produces two new free circles $C_{1}$ and $C_{2}$ in $\gamma(r)$.

(a) If $s(C)=+$, then $D_{\gamma, \rho}(r, s)=\left(\gamma(r), s_{+-}\right)+\left(\gamma(r), s_{-+}\right)$, where we define $s_{+-}\left(C_{1}\right)=+, s_{-}\left(C_{2}\right)=-$ and $s_{+-}(D)=s(D)$ for every other circle in $\operatorname{CIR}(r)$. We similarly define $s_{-+}$, with the roles of $C_{1}$ and $C_{2}$ reversed.

(b) If $s(C)=-$, then $D_{\gamma, \rho}(r, s)=\left(\gamma(r), s_{--}\right)$, where $s_{--}\left(C_{1}\right)=s_{--}\left(C_{2}\right)=-$ and $s_{--}(D)=s(D)$ for every other circle in $\operatorname{CIR}(r)$.

(3) Suppose $\gamma$ has both feet on the same arc $A$ in $\overleftarrow{H} \cap r$. Then $\gamma(r)$ will have a new free circle component $C$. Then $D_{\gamma, \rho}(r, s)=\left(\gamma(r), s^{\prime}\right)$, where $s^{\prime}(C)=-$ and $s^{\prime}(D)=s(D)$ for every other circle in $\operatorname{CIR}(\gamma(r))$. 
(4) If $\gamma$ has one foot on a cleaved circle $C$ and the other foot on a free circle $D$, then surgery on $\gamma$ will merge $D$ into $C$, leaving the other circles unchanged. If $s(D)=+$, then $D_{\gamma, \rho}(r, s)=\left(\gamma(r), s^{\prime}\right)$ with $s^{\prime}\left(C^{\prime}\right)=s\left(C^{\prime}\right)$ for every other circle in $\operatorname{CIR}(r)$, including $C$, while if $s(D)=-$ then $D_{\gamma, \rho}(r, s)=0$.

(5) In every case not covered on this list, $D_{\gamma, \rho}(r, s)=0$.

\subsection{Some classes of active bridges}

For a state $(r, s)$, we can use $s$ to group the bridges in $\operatorname{ACTIVE}(r)$ into (overlapping) classes:

(1) INTERIOR $(r, s)$ is the subset ACTIVE $(r)$ consisting of all $\gamma$ for which $D_{\gamma, \rho}(r, s)$ is nonzero. That is,

(a) both feet of $\gamma$ are on elements of $\operatorname{FREE}(r)$, or

(b) one foot of $\gamma$ is on $C \in \operatorname{cl}(r)$ and the other foot is on $C^{\prime} \in \operatorname{FREE}(r)$ with $s\left(C^{\prime}\right)=+$, or

(c) both feet are on the same arc of $C \cap \overleftarrow{\mathbb{H}}$ for some $C \in \operatorname{cl}(r)$

(2) $\operatorname{DEC}(r, s)$ is the subset $\operatorname{ACTIVE}(r)$ consisting of those $\gamma$ where

(a) both feet are on the same arc of $C \cap \overleftarrow{\mathbb{H}}$ for some $C \in \operatorname{cl}(r)$ with $s(C)=+$, or

(b) one foot of $\gamma$ is on $C \in \operatorname{cl}(r)$ with $s(C)=+$ and the other foot is on $C^{\prime} \in \operatorname{FREE}(r)$ with $s\left(C^{\prime}\right)=-$.

(3) $\overleftarrow{\mathrm{BR}}(r)$ is the subset $\operatorname{ACTIVE}(r)$ consisting of those $\gamma$ such that either

(a) $\gamma$ has one foot on $C_{1} \in \operatorname{cl}(r)$ and the other on a distinct circle $C_{2} \in \operatorname{cl}(r)$, or

(b) $\gamma$ has both feet on some $C \in \operatorname{cl}(r)$, but they are on different $\operatorname{arcs}$ of $C \cap \overleftarrow{\mathbb{H}}$.

If $r=(\rho, \vec{m})$ we will let $\operatorname{BRIDGE}(r)=\overleftarrow{\mathrm{BR}}(r) \cup \overrightarrow{\mathrm{BR}}(\vec{m})$ and $\overrightarrow{\mathrm{BR}}(r)=\overrightarrow{\mathrm{BR}}(\vec{m})$. There is a natural map BRIDGE $(r) \rightarrow \operatorname{BRIDGE}(\operatorname{cl}(r))$.

\section{The type $A$ structure for an inside tangle}

Given a diagram $\overleftarrow{T}$ for an inside tangle $\overleftarrow{\mathcal{T}}$, we describe a type $A$ structure on $\langle\overleftarrow{T} \rrbracket$ over $\mathcal{B} \Gamma_{n}$. This structure is specified by two bigrading preserving maps

$$
\begin{aligned}
& m_{1}:\langle\overleftarrow{T} \rrbracket \rightarrow\langle\overleftarrow{T} \rrbracket[(-1,0)], \\
& m_{2}:\left\langle\overleftarrow{T} \rrbracket \otimes_{\mathcal{I}} \mathcal{B} \Gamma_{n} \rightarrow\langle\overleftarrow{T} \rrbracket\right.
\end{aligned}
$$

Let $\xi=(r, s)$ be a generator of $\left\langle\overleftarrow{T} \rrbracket\right.$ with $\partial \xi=(L, \sigma)$, and let $e \in \mathcal{B} \Gamma_{n}$ be a generator. For $m_{1}$ we let $m_{1}(\xi)=d_{\text {APS }}(\xi)$, the differential on $\left\langle\overleftarrow{T} \rrbracket . d_{\text {APS }}\right.$ maps $(r, s)$ in bigrading $(h, q)$ to an element in $(h+1, q)$. This is bigrading preserving into $\langle\overleftarrow{T} \rrbracket[(-1,0)]$. 
To define the action $m_{2}$ we start by describing the action of the generators of $\mathcal{B} \Gamma_{n}$. Let $\xi=(r, s)$ be a generator of $\left\langle\overleftarrow{T} \rrbracket\right.$. Then $m_{2}\left(\xi \otimes_{\mathcal{I}} e\right)$ is computed as follows:

(1) When $e$ is an idempotent, $m_{2}\left(\xi \otimes I_{(L, \sigma)}\right)=\xi \cdot I_{(L, \sigma)}$ : the idempotent action defined above.

(2) When $e=\overrightarrow{e_{C}}$ for some $C \in \partial \xi$ with $\sigma(C)=+, m_{2}\left(\xi \otimes_{\mathcal{I}} \overrightarrow{e_{C}}\right)=\left(r, s_{C}\right)$, where $s_{C}(C)=-$ but equals $s$ on all other circles in $\operatorname{CIR}(r)$.

(3) When $e=\overleftarrow{e_{C}}$ for some $C \in \partial \xi$ with $\sigma(C)=+$,

$$
m_{2}\left(\xi \otimes_{\mathcal{I}} \overleftarrow{e_{C}}\right)=\sum_{\gamma \in \operatorname{DEC}((r, s), C)}(-1)^{I(r, \mathrm{CR}(\gamma))}\left(r_{\gamma}, s_{\gamma}\right)
$$

where $\operatorname{DEC}((r, s), C)$ are those active arcs which can change the decoration on $C, r_{\gamma}$ is the result of surgery on $\gamma$, and $s_{\gamma}$ is the new decoration with $s^{\prime}(C)=-\left(\right.$ and $s^{\prime}(D)=+$ if a new circle is created $)$.

(4) When $e=e_{\eta, \sigma, \sigma^{\prime}}$ for some $\eta \in \overleftarrow{\mathrm{BR}}(L)$

$$
m_{2}\left(\xi \otimes_{\mathcal{I}} e_{\eta, \sigma, \sigma^{\prime}}\right)=\sum_{\substack{\gamma \in \operatorname{ACTIVE}(r) \\ \mathrm{cl}(\gamma)=\eta}}(-1)^{I(r, \mathrm{CR}(\gamma))}\left(r_{\gamma}, s_{\gamma}\right),
$$

where $r_{\gamma}$ is surgery along $\gamma$ and $s_{\gamma}$ is the decoration on $r_{\gamma}$ which equals $s$ on $\operatorname{FREE}(r)$ and $\sigma^{\prime}$ on $\mathrm{cl}(r)$.

(5) When $e=e_{\eta, \sigma, \sigma^{\prime}}$ for some $\eta \in \overrightarrow{\mathrm{BR}}(L)$ and $r=(\rho, \vec{m})$, let $r^{\prime}=\left(\rho, \vec{m}_{\eta}\right)$ and $s^{\prime}$ equal $\sigma^{\prime}$ on the cleaved circles and $s$ on $\operatorname{FREE}\left(r^{\prime}\right), m_{2}\left(\xi \otimes_{\mathcal{I}} e_{\eta, \sigma, \sigma^{\prime}}\right)=\left(r^{\prime}, s^{\prime}\right)$.

(6) In all other cases $m_{2}\left(\xi \otimes_{\mathcal{I}} e\right)=0$. Note that $(r, s) \otimes_{\mathcal{I}} e_{1}=0$ unless $\partial(r, s)$ is the source of $e_{1}$ since otherwise $I_{\partial(r, s)} \cdot e_{1}=0$.

Proposition 4.1 The map $m_{2}$ constructed above is bigrading preserving.

Proof (1) This is immediate since the bigrading of $I_{(L, \sigma)}$ is $(0,0)$.

(2) When $e=\overrightarrow{e_{C}}$, if $\xi$ is in bigrading $(h, q)$, then $\xi \otimes_{\mathcal{I}} \overrightarrow{e_{C}}$ is in bigrading $(h, q)+$ $(0,-1)=(h, q-1)$, whereas $m_{2}\left(\xi \otimes_{\mathcal{I}} \overrightarrow{e_{C}}\right)$ is in bigrading $(h, q-1)$ since we changed a $\left(0,+\frac{1}{2}\right)$ cleaved circle to a $\left(0,-\frac{1}{2}\right)$ cleaved circle.

(3) When $e=\overleftarrow{e_{C}}$, the bigrading of $\xi \otimes_{\mathcal{I}} \overleftarrow{e_{C}}$ is $(h, q)+(1,1)$. For $m_{2}\left(\xi \otimes_{\mathcal{I}} \overleftarrow{e_{C}}\right)$ we consider the bigrading in two cases. If we merge a - free circle, then $q=\bar{q}+\frac{1}{2}-1$ and the bigrading of $\left(r_{\gamma}, s_{\gamma}\right)$ is $\left(h, \bar{q}-\frac{1}{2}\right)+(1,1)=(h+1, q+1)$, where the additional $(1,1)$ comes from the grading shifts associated with $1-$ resolving a crossing. If we divide $\mathrm{a}+$ cleaved circle then the bigrading change is from $\left(h, \bar{q}+\frac{1}{2}\right)+(1,1)=\left(h+1, q+\frac{3}{2}\right)$ to $\left(h, \bar{q}+1-\frac{1}{2}\right)+(1,1)=\left(h+1, q+\frac{3}{2}\right)$. In either case, there is a $(0,0)$ change in bigrading. 
(4) When $e=e_{\eta, \sigma, \sigma^{\prime}}$ for some $\eta \in \overleftarrow{\mathrm{BR}}(L)$, if $\eta$ merges two plus circles then $\xi \otimes_{\mathcal{I}} e_{\eta, \sigma, \sigma^{\prime}}$ has bigrading $\left(h, \bar{q}+\frac{1}{2}+\frac{1}{2}\right)+\left(1, \frac{1}{2}\right)=\left(h+1, \bar{q}+\frac{3}{2}\right)$, while $m_{2}\left(\xi \otimes_{\mathcal{I}} e_{\eta, \sigma, \sigma^{\prime}}\right)$ has $\left(h, \bar{q}+\frac{1}{2}\right)+(1,1)$, since we change the resolution at a crossing. If $\eta$ merges a + and a - we have $\left(h, \bar{q}+\frac{1}{2}-\frac{1}{2}\right)+\left(1, \frac{1}{2}\right)=\left(h+1, \bar{q}+\frac{1}{2}\right)$ before and $\left(h, \bar{q}-\frac{1}{2}\right)+(1,1)$ after. If $\eta$ divides a + circle then we start with $\left(h, \bar{q}+\frac{1}{2}\right)+\left(1, \frac{1}{2}\right)$ and end with $\left(h, \bar{q}+\frac{1}{2}-\frac{1}{2}\right)+(1,1)$, while if $\eta$ divides a - circle we start with $\left(h, \bar{q}-\frac{1}{2}\right)+\left(1, \frac{1}{2}\right)$ and end with $\left(h, \bar{q}-\frac{1}{2}-\frac{1}{2}\right)+(1,1)$. Each of these is a $(0,0)$ change.

(5) When $e=e_{\eta, \sigma, \sigma^{\prime}}$ for some $\eta \in \overrightarrow{\mathrm{BR}}(L)$ and $r=(\rho, \vec{m})$, if surgery on $\eta$ merges two + cleaved circles, then the bigrading of $\xi \otimes_{\mathcal{I}} e_{\eta, \sigma, \sigma^{\prime}}$ is $\left(h, \bar{q}+\frac{1}{2}+\frac{1}{2}\right)+\left(0,-\frac{1}{2}\right)$, while that of $m_{2}\left(\xi \otimes_{\mathcal{I}} e_{\eta, \sigma, \sigma^{\prime}}\right)$ is $\left(h, \bar{q}+\frac{1}{2}\right)$ (as there is no crossing change). Likewise for a + and - circle: $\left(h, \bar{q}+\frac{1}{2}-\frac{1}{2}\right)+\left(0,-\frac{1}{2}\right) \rightarrow\left(h, \bar{q}-\frac{1}{2}\right)$, while for a divide of a circle: $\left(h, \bar{q}+\frac{1}{2}\right)+\left(0,-\frac{1}{2}\right) \rightarrow\left(h, \bar{q}+\frac{1}{2}-\frac{1}{2}\right)$, and a divide of a - circle: $\left(h, \bar{q}-\frac{1}{2}\right)+\left(0,-\frac{1}{2}\right) \rightarrow\left(h, \bar{q}-\frac{1}{2}-\frac{1}{2}\right)$. In all cases there is a $(0,0)$ change in bigrading.

(6) This is true by definition true since the image of $m_{2}$ is 0 .

This specifies $m_{2}$ on the generators of $\mathcal{B} \Gamma_{n}$. To define $m_{2}$ on all elements, we impose the following relation: if $p_{1}, p_{2} \in \mathcal{B} \Gamma_{n}$, we define

$$
m_{2}\left(\xi \otimes p_{1} p_{2}\right)=m_{2}\left(m_{2}\left(\xi \otimes p_{1}\right) \otimes p_{2}\right) .
$$

Proposition 4.2 If two products of the generators $p_{1}$ and $p_{2}$ define equal elements in $\mathcal{B} \Gamma_{n}$, then $m_{2}\left(\xi \otimes p_{1}\right)=m_{2}\left(\xi \otimes p_{2}\right)$ for every $\xi \in\langle\overleftarrow{T} \rrbracket$.

Thus the rules above fully specify $m_{2}:\left\langle\overleftarrow{T} \rrbracket \otimes_{\mathcal{I}} \mathcal{B} \Gamma_{n} \rightarrow\langle\overleftarrow{T} \rrbracket\right.$

Proof It suffices to show that $m_{2}(\xi \otimes \rho)=0$ whenever $\rho$ is a relation defining $\mathcal{B} \Gamma_{n}$. We start with the relations from disjoint supports. Suppose $C$ and $D$ are distinct cleaved circles with $\sigma(C)=\sigma(D)=+$. Then

(17) $m_{2}\left(\xi \otimes\left(\overrightarrow{e_{C}} \overrightarrow{e_{D}}-\overrightarrow{e_{D}} \overrightarrow{e_{C}}\right)\right)=m_{2}\left(m_{2}\left(\xi \otimes \overrightarrow{e_{C}}\right) \otimes \overrightarrow{e_{D}}\right)-m_{2}\left(m_{2}\left(\xi \otimes \overrightarrow{e_{D}}\right) \otimes \overrightarrow{e_{C}}\right)$

$$
=\left(r, s_{C, D}\right)-\left(r, s_{C, D}\right)=0 \text {. }
$$

On the other hand,

$$
\begin{aligned}
m_{2} & \left(\xi \otimes\left(\overleftarrow{e_{C}} \overrightarrow{e_{D}}-\overrightarrow{e_{D}} \overleftarrow{e_{C}}\right)\right) \\
& =m_{2}\left(m_{2}\left(\xi \otimes \overleftrightarrow{e_{C}}\right) \otimes \overrightarrow{e_{D}}\right)-m_{2}\left(m_{2}\left(\xi \otimes \overrightarrow{e_{D}}\right) \otimes \overleftrightarrow{e_{C}}\right) \\
& =\sum_{\gamma \in \operatorname{DEC}((r, s), C)}(-1)^{I(r, \mathrm{CR}(\gamma))}\left(r_{\gamma}, s_{\gamma, D}\right)-\sum_{\gamma \in \operatorname{DEC}((r, s), C)}(-1)^{I(r, \mathrm{CR}(\gamma))}\left(r_{\gamma}, s_{D, \gamma}\right) \\
& =0
\end{aligned}
$$


To compute $m_{2}\left(\xi \otimes\left(\overleftarrow{e_{C}} \overleftarrow{e_{D}}+\overleftarrow{e_{D}} \overleftarrow{e_{C}}\right)\right)$ note that each $m_{2}\left(m_{2}\left(\xi \otimes \overleftarrow{e_{C}}\right) \otimes \overleftarrow{e_{D}}\right)$ and $m_{2}\left(m_{2}\left(\xi \otimes \overleftarrow{e_{D}}\right) \otimes \overleftarrow{e_{C}}\right)$ are sums over pairs of edges $\gamma \in \operatorname{DEC}(r, s, C)$ and $\gamma^{\prime} \in$ $\operatorname{DEC}(r, s, D)$. In one case we sum over $\left(\gamma, \gamma^{\prime}\right)$ pairs and in the other $\left(\gamma^{\prime}, \gamma\right)$ pairs. In each case we obtain $\left(r_{\gamma, \gamma^{\prime}}, s_{\gamma, \gamma^{\prime}}\right)$ with $s_{\gamma, \gamma^{\prime}}$ uniquely determined by the requirement that $C$ and $D$ are decorated with - . Thus we need only look at the signs: for $\left(\gamma, \gamma^{\prime}\right)$ we have $(-1)^{I(r, \mathrm{CR}(\gamma))+I\left(r_{\gamma}, \mathrm{CR}\left(\gamma^{\prime}\right)\right)}$ which is $-(-1)^{I\left(r, \mathrm{CR}\left(\gamma^{\prime}\right)\right)+I\left(r_{\gamma^{\prime}}, \mathrm{CR}(\gamma)\right)}$. Consequently, they cancel in the sum.

Now suppose that $C_{1}$ and $C_{2}$ are cleaved circles in $r$ with $s\left(C_{1}\right)=s\left(C_{2}\right)=+$. Let $\beta$ be an active arc which merges $C_{1}$ and $C_{2}$ to get $C$ and maps to $\gamma \in \overleftarrow{\mathrm{BR}}(L)$. We can partition the active arcs $\alpha$ which contribute to $\operatorname{DEC}\left(r_{\beta}, s_{\beta}, C\right)$ into the three sets: $\operatorname{DEC}\left(r, s, C_{1}\right), \operatorname{DEC}\left(r, s, C_{2}\right)$, and $\alpha$ which also map to $\gamma$. To obtain $m_{2}\left(\xi \otimes m_{\gamma} \overleftarrow{e_{C}}\right)$ we sum over all such $\beta$ and $\alpha$ arcs: $\sum_{(\beta, \alpha)}(-1)^{I(\beta)+I\left(r_{\beta}, \alpha\right)}\left(r_{\beta, \alpha}, s_{\beta \alpha}\right)$. For $\alpha$ isotopic as a bridge to $\beta$ the term for $(\alpha, \beta)$ occurs in this sum, with sign $(-1)^{I(\alpha)+I\left(r_{\alpha}, \beta\right)}$. This cancels the term from $(\beta, \alpha)$. Thus

$$
\begin{aligned}
& m_{2}\left(\xi \otimes m_{\gamma} \stackrel{\leftarrow}{e_{C}}\right)=\sum_{\beta, \alpha \in \operatorname{DEC}\left(r, s, C_{1}\right)}(-1)^{I(\beta)+I\left(r_{\beta}, \alpha\right)}\left(r_{\beta, \alpha}, s_{\beta \alpha}\right) \\
& +\sum_{\beta, \alpha \text { DEC }\left(r, s, C_{2}\right)}(-1)^{I(\beta)+I\left(r_{\beta}, \alpha\right)}\left(r_{\beta, \alpha}, s_{\beta \alpha}\right) \\
& =-\sum_{\alpha \in \mathrm{DEC}\left(r, s, C_{1}\right), \beta}(-1)^{I(\alpha)+I\left(r_{\alpha}, \beta\right)}\left(r_{\alpha, \beta}, s_{\alpha \beta}\right) \\
& -\sum_{\alpha \in \mathrm{DEC}\left(r, s, C_{2}\right), \beta}(-1)^{I(\alpha)+I\left(r_{\alpha}, \beta\right)}\left(r_{\alpha, \beta}, s_{\alpha \beta}\right)
\end{aligned}
$$

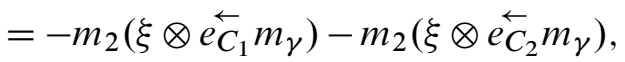

which verifies that $m_{2}$ is compatible with relation (11). Exactly the same argument applies to for $\gamma \in \overrightarrow{\mathrm{BR}}(L)$, although we no longer need to sum over the representatives of $\gamma$ since there is only one such bridge. More significantly, all the terms occur with sign $(-1)^{I(\alpha)}$ since surgery on $\gamma$ does not affect the signs. The conclusion becomes

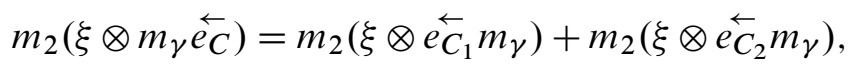

which is compatible with relation (9). The case where surgery on $\gamma$ is divisive follows from the same line of reasoning.

For $\overrightarrow{e_{C}}$ and $\gamma$ merging $C_{1}$ and $C_{2}$ the situation is easier. First, suppose $\gamma \in \overrightarrow{\mathrm{BR}}(L)$. Then

$$
m_{2}\left(\xi \otimes m_{\gamma} \overrightarrow{e_{C}}\right)=\left(r, s_{\gamma, C}\right)
$$

while

$$
m_{2}\left(\xi \otimes \overrightarrow{e_{C_{1}}} m_{\gamma}\right)=m_{2}\left(\left(r, s_{C_{1}}\right) \otimes m_{\gamma}\right)=\left(r, s_{C, \gamma}\right)
$$


As these are equal, and as $C_{2}$ plays a symmetric role, $m_{2}$ is compatible with this type of relation. Again, the case for dividing is similar. For $\overrightarrow{e_{C}}$ and $\gamma \in \overleftrightarrow{\mathrm{BR}}(L)$, we have

$$
m_{2}\left(\xi \otimes m_{\gamma} \overrightarrow{e_{C}}\right)=\sum_{\alpha}(-1)^{I(\alpha)}\left(r_{\alpha}, s_{\alpha, C}\right),
$$

where the sum is over active arcs which map to $\gamma$. On the other hand, while

$$
m_{2}\left(\xi \otimes \vec{e}_{1} m_{\gamma}\right)=m_{2}\left(\left(r, s_{C_{1}}\right) \otimes m_{\gamma}\right)=\sum_{\alpha}(-1)^{I(\alpha)}\left(r_{\alpha}, s_{C, \alpha}\right) .
$$

Thus $m_{2}$ is compatible with $m_{\gamma} \overrightarrow{e_{C}}=\vec{e}_{C_{1}} m_{\gamma}$ for all $\gamma \in \operatorname{BRIDGE}(L)$.

Suppose that $\gamma$ and $\gamma^{\prime}$ are in $\operatorname{BRIDGE}(L)$ and that there is a commuting square:

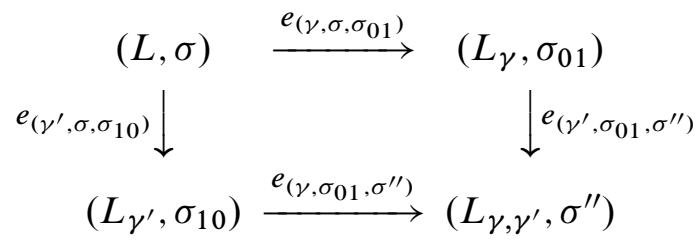

Then we have the following cases:

(1) Both $\gamma$ and $\gamma^{\prime}$ are in $\overrightarrow{\mathrm{BR}}(L)$ we need to see $m_{2}\left(\xi \otimes\left(e_{\gamma} e_{\gamma^{\prime}}-e_{\gamma^{\prime}} e_{\gamma}\right)\right)=0$. However, both terms resulting from expanding $m$ will equal $\left(r_{\gamma \gamma^{\prime}}, s^{\prime \prime}\right)$, where $r_{\gamma, \gamma^{\prime}}$ is identical to $r$ in $\overleftarrow{\mathbb{H}}$ but equals $L_{\gamma \gamma^{\prime}}$ in $\overrightarrow{\mathbb{H}}$, and $s^{\prime \prime}$ is $s$ on $\operatorname{FREE}(r)$ but $\sigma^{\prime \prime}$ on $\operatorname{cl}(r)$. Since both terms are identical, the difference will be zero and $m_{2}$ is compatible with this case.

(2) If $\gamma$ in $\overleftarrow{\mathrm{BR}}(L)$ but $\gamma^{\prime} \in \overrightarrow{\mathrm{BR}}(L)$, then we need $m_{2}\left(\xi \otimes\left(e_{\gamma} e_{\gamma^{\prime}}-e_{\gamma^{\prime}} e_{\gamma}\right)\right)=0$. The action of $e_{\gamma}$ followed by $e_{\gamma^{\prime}}$ (or vice versa) will give $\sum_{\alpha}(-1)^{I(r, \alpha)}\left(r_{\alpha, \gamma^{\prime}}, s^{\prime \prime}\right)$, where the sum is over all active arcs for $r$ which have image $\gamma$ in $\operatorname{cl}(r)$. Since surgery on $\gamma^{\prime}$ does not affect the sign, we see that the two terms will cancel, and $m_{2}$ is compatible with this case.

(3) Suppose both $\gamma$ and $\gamma^{\prime}$ are in $\overleftarrow{\mathrm{BR}}(L)$, then $m_{2}\left(\xi \otimes e_{\gamma} e_{\gamma^{\prime}}\right)$ is the sum over pairs of active arcs $\left(\alpha, \alpha^{\prime}\right)$ for $r$ which map to $\gamma$ and $\gamma^{\prime}$ when considered as bridges. Each pair also contributes to $m_{2}\left(\xi \otimes e_{\gamma^{\prime}} e_{\gamma}\right)$ but in the reversed order $\left(\alpha^{\prime}, \alpha\right)$. The decorations of the result are determined by $\sigma^{\prime \prime}$, so we need only check the signs of each term. The sign for $\left(\alpha, \alpha^{\prime}\right)$ is $(-1)^{I(r, \mathrm{CR}(\alpha))+I\left(r_{\alpha}, \mathrm{CR}\left(\alpha^{\prime}\right)\right)}$ while that for $\left(\alpha^{\prime}, \alpha\right)$ is $(-1)^{I\left(r, \mathrm{CR}\left(\alpha^{\prime}\right)\right)+I\left(r_{\alpha^{\prime}}, \mathrm{CR}(\alpha)\right)}$. Due to the ordering of the crossings, one of these will be +1 and the other -1 . Consequently, $m_{2}\left(\xi \otimes e_{\gamma} e_{\gamma^{\prime}}\right)=-m_{2}\left(\xi \otimes e_{\gamma^{\prime}} e_{\gamma}\right)$ which is compatible with the relation for $\stackrel{\mathrm{BR}}{\leftarrow}(L)$. 
Note that a similar argument to that in (1) works for all pairs of right bridge edges that form commutative squares, so it will be omitted. For left bridge elements, there are two further cases to consider:

(a) Suppose $\delta \in \overleftarrow{B}_{o}(L, \gamma)$ and $\delta_{1}, \delta_{2} \in B_{S}\left(L_{\gamma}, \gamma^{\dagger}\right)$ map to $\delta$ under surgery along $\gamma^{\dagger}$ Likewise, suppose $\gamma_{1}$ and $\gamma_{2}$ map to $\gamma$ under surgery along $\delta^{\dagger}$. If we orient the mutual arc for $\delta$ and $\gamma$, then $\delta_{1}$ corresponds to the version of $\delta$ before $\gamma$ along the mutual arc, and $\delta_{2}$ corresponds to that after $\gamma$. Likewise for $\gamma_{i}, i=1,2$ and $\delta$. There can then be (anti-)commutative squares

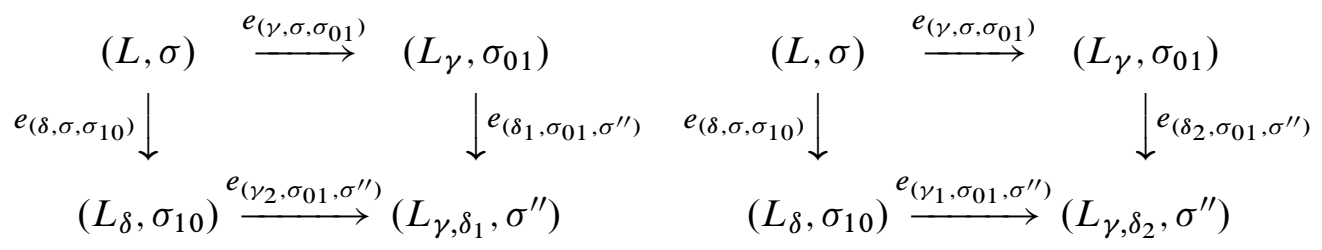

where $L_{\gamma, \delta_{2}}$ and $L_{\gamma, \delta_{1}}$ have different left matchings. Two resolution $\operatorname{arcs} a_{1}$ and $a_{2}$ for $\xi$, one corresponding to $\gamma$ and one corresponding to $\delta$, will be counted in the action of either $\gamma$ followed by $\delta_{1}$, or $\gamma$ followed by $\delta_{2}$ (but not both). Reversing the order means contributing to $\delta$ followed by $\gamma_{2}$, or $\delta$ followed by $\gamma_{1}$. As these contribute with the usual sign conventions, the contributions of the pair will cancel in either the action of $e_{\gamma} e_{\delta_{1}}+e_{\delta} e_{\gamma_{2}}$ or $e_{\gamma} e_{\delta_{2}}+e_{\delta} e_{\gamma_{1}}$, which verifies that the action respects the anti-commutativity in this case.

(b) Now suppose that $\overleftarrow{\alpha}$ is in $B_{S}(L, \overleftarrow{\gamma})$ and that $\overleftarrow{\beta}$ is the bridge obtained by sliding $\overleftarrow{\alpha}$ over $\overleftarrow{\gamma}$. Let $\overleftarrow{\delta}$ be the image of $\overleftarrow{\alpha}$ and $\overleftarrow{\beta}$ in $L \overleftarrow{\gamma}, \overleftarrow{\zeta}$ be the image of $\overleftarrow{\alpha}$ and $\overleftarrow{\gamma}$ in $L \overleftarrow{\beta}$ and $\overleftarrow{\eta}$ be the image of $\overleftarrow{\beta}$ and $\overleftarrow{\gamma}$ in $L_{\alpha} \overleftarrow{\alpha}$. The action of $\overleftarrow{e_{\alpha}} \overleftarrow{e_{\eta}}$ is the sum over active resolution arcs $a_{1}$ and $a_{2}$ for $\xi$ with $a_{1}$ representing $\overleftarrow{\alpha}$ and $a_{2}$ representing $\overleftarrow{\eta}$ in $L_{\alpha}$ Thus, $a_{2}$ represents one of $\beta$ or $\gamma$ in $L$. Reversing the order thus gives a contribution to either $\overleftarrow{e_{\beta}} \overleftarrow{e_{\zeta}}$ or $\overleftarrow{e_{\gamma}} \overleftarrow{e_{\delta}}$. However, pairs representing $\beta$ and $\gamma$ also contribute to $\overleftarrow{e_{\beta}} \overleftarrow{e_{\zeta}}$, while their reverse contributes to $\overleftarrow{e_{\gamma}} \overleftarrow{e_{\delta}}$. Thus, if we consider all ordered pairs $\left(a_{1}, a_{2}\right)$ of resolution arcs which represent pairs of $\alpha, \beta$, or $\gamma$, in either order, we will count both $\left(a_{1}, a_{2}\right)$ and $\left(a_{2}, a_{1}\right)$ for each such pair, and they will contribute with opposite signs (due to the Khovanov sign conventions) in the action of $\overleftarrow{e_{\alpha}} \overleftarrow{e_{\eta}}+\overleftarrow{e_{\beta}} \overleftarrow{e_{\zeta}}+\overleftarrow{e_{\gamma}} \overleftarrow{e_{\delta}}$ on $\xi$ Consequently, all the terms in this action will cancel, verifying that it acts as 0 .

For the additional bridge relations, suppose $\gamma \in \overrightarrow{\mathrm{BR}}(L)$. Then there is a relation $e_{\gamma, \sigma, \sigma^{\prime}} e_{\gamma^{\dagger} \sigma^{\prime}, \sigma_{C}}=\overrightarrow{e_{C}}$. In this case

$$
\begin{aligned}
m_{2}\left(\xi \otimes\left(e_{\gamma, \sigma, \sigma^{\prime}} e_{\gamma^{\dagger} \sigma^{\prime}, \sigma_{C}}-\vec{e}_{C}\right)\right) & =\left(\left(\rho, \vec{m}_{\gamma \gamma^{\dagger}}\right), s_{C}\right)-\left(r, s_{C}\right) \\
& =\left((\rho, \vec{m}), s_{C}\right)-\left(r, s_{C}\right)=\left(r, s_{C}\right)-\left(r, s_{C}\right)=0,
\end{aligned}
$$


where $\vec{m}_{\gamma \gamma^{\dagger}}=\vec{m}$ follows from the result that surgery on a bridge $\gamma$ for $L$, followed by surgery on $\gamma^{\dagger}$, recovers $L$.

Now suppose that $\gamma \in \overleftarrow{\mathrm{BR}}(L)$ and $\eta \in \overleftarrow{\mathrm{BR}}\left(L_{\gamma}\right)$ intersects $\gamma^{\dagger}$ non-trivially. We need to see that $m_{2}\left(\xi \otimes e_{\gamma} e_{\eta}\right)=0$ since $e_{\gamma} e_{\eta}=0$. However, in $m_{2}\left(m_{2}\left(\xi \otimes e_{\gamma}\right) \otimes e_{\eta}\right)$ the action of $e_{\eta}$ will result in a sum over active arcs in $r_{\gamma}$ which map to $\eta$ in $\operatorname{cl}(r)$. Each active arc comes from a crossing, and thus must already be present in $r$ for it to be present in $r_{\gamma}$. This excludes there being any active arc for $\eta$ in $r_{\gamma}$. Consequently the sum is 0 and we have verified that $m_{2}$ is compatible with this relation.

Since $m_{2}(\xi \otimes R)=0$ whenever $R$ is a relation for $\mathcal{B} \Gamma_{n}$, we have shown that $m_{2}$ is well defined.

Proposition 4.3 For $\xi=(r, s)$ a generator of $\left\langle\overleftarrow{T} \rrbracket\right.$ and $\rho_{1}, \rho_{2} \in \mathcal{B} \Gamma_{n}$, the maps $m_{1}$ and $m_{2}$ above satisfy the following properties:

$$
\begin{aligned}
& 0=m_{1}\left(m_{1}(\xi)\right), \\
& 0=(-1)^{\overleftarrow{l}\left(\rho_{1}\right)} m_{2}\left(m_{1}(\xi) \otimes \rho_{1}\right)+m_{2}\left(\xi \otimes d_{\Gamma}\left(\rho_{1}\right)\right)-m_{1}\left(m_{2}\left(\xi \otimes \rho_{1}\right)\right), \\
& 0=m_{2}\left(m_{2}\left(\xi \otimes \rho_{1}\right) \otimes \rho_{2}\right)-m_{2}\left(\xi \otimes \rho_{1} \rho_{2}\right) .
\end{aligned}
$$

Note These are the relations for $\left\langle\overleftarrow{T} \rrbracket\right.$ to be an $A_{\infty}$-module over the differential graded algebra $\mathcal{B} \Gamma_{n}$, as in [8], with $m_{n}=0$ for $n \geq 3$.

Proof That $m_{1}\left(m_{1}(\xi)\right)=0$ is a byproduct of $m_{1}=d$ being a differential (see also the proof that $\vec{\delta}$ is a $D$ structure for an outside tangle). Furthermore, that $m_{2}$ defines a right action follows from how we bootstrapped $m_{2}$ from the definition on generators. Thus, we need only verify that $d$ and $m_{2}$ are compatible with $\mu_{\Gamma}$ through the equation

$$
d\left(m_{2}\left(\xi \otimes \rho_{1}\right)\right)=(-1)^{\overleftarrow{l}\left(\rho_{1}\right)} m_{2}\left(d(\xi) \otimes \rho_{1}\right)+m_{2}\left(\xi \otimes d_{\Gamma}\left(\rho_{1}\right)\right)
$$

It suffices to prove this for $\rho_{1}$ of length 0 or 1 since we can bootstrap the relation for longer words using

$$
\begin{aligned}
d(\xi \cdot(\alpha \beta)) & =d((\xi \cdot \alpha) \cdot \beta) \\
& =(-1)^{\overleftarrow{l}(\beta)} d(\xi \cdot \alpha) \cdot \beta+(\xi \cdot \alpha) \cdot d_{\Gamma}(\beta) \\
& =(-1)^{\overleftarrow{l}(\beta)+\overleftarrow{l}(\alpha)}(d(\xi) \cdot \alpha) \cdot \beta+(-1)^{\overleftarrow{l}(\beta)}\left(\xi \cdot d_{\Gamma}(\alpha)\right) \cdot \beta+\xi \cdot\left(\alpha d_{\Gamma}(\beta)\right) \\
& =(-1)^{\overleftarrow{l}(\beta)+\overleftarrow{l}(\alpha)}[d(\xi) \cdot(\alpha \beta)]+\xi \cdot\left[(-1)^{\overleftarrow{l}(\beta)} d_{\Gamma}(\alpha) \beta+\alpha d_{\Gamma}(\beta)\right] \\
& =(-1)^{\overleftarrow{l}(\beta)+\overleftarrow{l}(\alpha)}\left[d(\xi) \cdot(\alpha \beta)+\xi \cdot d_{\Gamma}(\alpha \beta)\right] .
\end{aligned}
$$

For length 0 , we have $\rho_{1}=I_{(L, \sigma)}$ for some idempotent. If $\partial \xi \neq(L, \sigma)$ then both sides are zero since $m_{2}\left(\xi \otimes I_{(L, \sigma)}\right)=m_{2}(0)=0, d(\xi)$ has the same boundary as $\xi$ 
so $d(\xi) \otimes I_{(L, \sigma)}=0$, and $d_{\Gamma}\left(I_{(L, \sigma)}\right)=0$ for every idempotent. On the other hand, if $\partial \xi=(L, \sigma)$ the last term still vanishes, and

$$
d\left(m_{2}\left(\xi \otimes I_{(L, \sigma)}\right)\right)=d(\xi)=m_{2}\left(d(\xi) \otimes I_{(L, \sigma)}\right) .
$$

For length-one words, we need to check when $\rho_{1}=\overrightarrow{e_{C}}, \overleftarrow{e_{C}}$, or $e_{\gamma}$ for $\gamma \in \operatorname{BRIDGE}(L)$, where $\partial \xi=(L, \sigma)$. We know $d_{\Gamma}\left(\overrightarrow{e_{C}}\right)=0$ and $\overleftarrow{l}\left(\overrightarrow{e_{C}}\right)=0$, so for $\overrightarrow{e_{C}}$ we need only verify that $d\left(m_{2}\left(\xi \otimes \overrightarrow{e_{C}}\right)\right)=m_{2}\left(d(\xi) \otimes \overrightarrow{e_{C}}\right)$. If $\xi$ has $\sigma(C)=-$ then both are 0 , whereas if $\sigma(C)=+$ then both equal $\Sigma_{\alpha}(-1)^{I(\alpha)}\left(r_{\alpha}, s_{\alpha, C}\right)$, where the sum is over all active, non-bridging arcs $\alpha$ and $s_{\alpha, C}$ is any decoration compatible with $d, r_{\alpha}$, and assigning $C$ a - . For $e_{\gamma}$ with $\gamma \in \overrightarrow{\mathrm{BR}}(L)$, the only difference is that the sum is over terms $\left(r_{\alpha, \gamma}, s_{\alpha, \gamma}\right)$.

For $e_{\gamma}$ with $\gamma \in \overleftarrow{\mathrm{BR}}(L), d_{\Gamma}$ still vanishes but $\overleftarrow{l}=1$. We then have

$$
d\left(m_{2}\left(\xi \otimes e_{\gamma}\right)\right)=\sum_{\alpha, \beta}(-1)^{I(r, \alpha)+I\left(r_{\alpha}, \beta\right)}\left(r_{\alpha, \beta}, s_{\alpha, \beta}\right),
$$

where the sum is over all active $\operatorname{arcs} \alpha$ which map to $\gamma$ and all active $\operatorname{arcs} \beta$ which contribute to $d$ (as well as all compatible decorations on $r_{\alpha, \beta}$. On the other hand,

$$
m_{2}\left(d(\xi) \otimes e_{\gamma}\right)=\sum_{\beta, \alpha}(-1)^{I(r, \beta)+I\left(r_{\beta}, \alpha\right)}\left(r_{\beta, \alpha}, s_{\beta, \alpha}\right)
$$

due to the ordering of the crossings the signs will be different for each $(\alpha, \beta)$ term, so

$$
d\left(m_{2}\left(\xi \otimes e_{\gamma}\right)\right)=-m_{2}\left(d(\xi) \otimes e_{\gamma}\right)=(-1)^{\overleftarrow{l}\left(e_{\gamma}\right)} m_{2}\left(d(\xi) \otimes e_{\gamma}\right)
$$

We are left with verifying the formula for $\overleftarrow{e_{C}}$. We start with

$$
d\left(m_{2}\left(\xi \otimes \overleftrightarrow{e_{C}}\right)\right)=\sum_{\alpha, \beta}(-1)^{I(r, \alpha)+I\left(r_{\alpha}, \beta\right)}\left(r_{\alpha, \beta}, s_{\alpha, \beta}\right),
$$

where the sum is over all active $\operatorname{arcs} \alpha \in \operatorname{DEC}(r, s, C)$ and $\beta$ contributing to $d$ on $r_{\alpha}$. Furthermore,

$$
m_{2}\left(d(\xi) \otimes \overleftrightarrow{e_{C}}\right)=\sum_{\beta^{\prime}, \alpha^{\prime}}(-1)^{I\left(r, \beta^{\prime}\right)+I\left(r_{\beta^{\prime}}, \alpha^{\prime}\right)}\left(r_{\beta^{\prime}, \alpha^{\prime}}, s_{\beta^{\prime}, \alpha^{\prime}}\right)
$$

where the sum is over all $\beta^{\prime}$ contributing to $d$ on $r$ and over all $\alpha^{\prime}$ contributing to $\operatorname{DEC}\left(r_{\beta^{\prime}}, s_{\beta^{\prime}}, C\right)$.

Some ordered pairs $(\alpha, \beta)$ contribute a term to $d\left(m_{2}\left(\xi \otimes \overleftrightarrow{e_{C}}\right)\right)$ while the pair $(\beta, \alpha)$ contributes to $m_{2}\left(d(\xi) \otimes \overleftarrow{e_{C}}\right)$. The corresponding terms in the sum occur with coefficients that are equal but have opposite signs, and thus cancel. We let $R$ be the sum over the pairs $(\alpha, \beta)$ in $d\left(m_{2}\left(\xi \otimes \overleftarrow{e_{C}}\right)\right)$, and define $\Psi_{1}=d\left(m_{2}\left(\xi \otimes \overleftarrow{e_{C}}\right)\right)-R$. 
Then $m_{2}\left(d(\xi) \otimes \overleftarrow{e_{C}}\right)=-R+\Psi_{2}$ for some $\Psi_{2}$. We can characterize the terms which contribute to $\Psi_{1}$ and $\Psi_{2}$. These terms correspond to pairs $(\alpha, \beta)$ coming from bridges $\gamma, \gamma^{\dagger}$ with $C$ as their active circle. A sign check, however, shows that $\Psi_{1}+\Psi_{2}=m_{2}\left(\xi \otimes \sum_{\gamma} e_{\gamma} e_{\gamma^{\dagger}}\right)$, where the sum is over all $\gamma$ with active circle $C$. As this sum is just the action of $-d_{\Gamma}\left(\overleftarrow{e_{C}}\right)$ we obtain the relation

$$
\begin{aligned}
& d\left(m_{2}\left(\xi \otimes \overleftarrow{e_{C}}\right)\right)=R+\Psi_{1} \\
& =-\left(-R+\Psi_{2}\right)+\Psi_{2}+\Psi_{1} \\
& =-m_{2}\left(d(\xi) \otimes \overleftarrow{e_{C}}\right)+\left(\Psi_{1}+\Psi_{2}\right) \\
& =-m_{2}\left(d(\xi) \otimes \overleftarrow{e_{C}}\right)-m_{2}\left(\xi \otimes-d_{\Gamma}\left(\overleftarrow{e_{C}}\right)\right)
\end{aligned}
$$

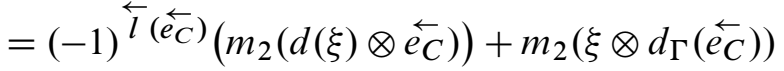

as required.

We have now verified that the action of the length one words is compatible with the (right) Leibniz relation, and thus using the bootstrap, that $d$ is a (right) differential on the module $\langle\overleftarrow{T} \rrbracket$

\section{Background on type $A$ structures and their simplification}

\subsection{Algebra}

In this section we redefine $A_{\infty}$-algebras and module to be consistent with our sign conventions. We begin with some notation for handling signs and gradings

Definition 5.1 Let $W=W_{0} \oplus W_{1}$ be a $\mathbb{Z} / 2 \mathbb{Z}$-graded module. $\left|\mathbb{I}_{W}\right|: W \rightarrow W$ is the signed identity defined by linearly extending

$$
\left|\mathbb{I}_{W}\right|(w)=(-1)^{\operatorname{gr}(w)} w
$$

for homogeneous $w \in A$.

Note $\operatorname{By}|\mathbb{I}|^{j}$ we mean the composition of $|\mathbb{I}|$ with itself $j$ times. Furthermore, by $|\mathbb{I}|^{j \otimes n}$ we will mean the function $|\mathbb{I}|^{j} \otimes \cdots \otimes|\mathbb{I}|^{j}$, where there are $n$ factors. For an element $\alpha,|\mathbb{I}|^{j}(\alpha)$ will be shortened to $|\alpha|^{j}$. Thus, on a homogeneous element $\alpha$, $|\alpha|^{j}=(-1)^{j \operatorname{gr}(\alpha)} \alpha$, and $\left.\left.|| \alpha\right|^{j}\right|^{k}=|\alpha|^{j+k}$.

Definition 5.2 An $A_{\infty}$-algebra $A$ over a ring $R$ is a graded module $A$ equipped with maps $\mu_{n}: A^{\otimes n} \rightarrow A[n-2]$ for each $n \in \mathbb{N}$ which satisfy the relation

$$
0=\sum_{\substack{i+j=n+1 \\ k \in\{1, \ldots, n-j+1\}}}(-1)^{j(i+1)+(k+1)(j+1)} \mu_{i}\left(\mathbb{I}^{\otimes(k-1)} \otimes \mu_{j} \otimes|\mathbb{I}|^{j \otimes(n-k-j+1)}\right) .
$$


Definition 5.3 A right module over a $\mathbb{Z} / 2 \mathbb{Z}$-graded differential $R$-algebra $\left(A, \mu_{1}, \mu_{2}\right)$ is an $R$-module $M$ together with maps $m_{1}: M \rightarrow M[-1]$ and $m_{2}: M \otimes_{R} A \rightarrow M$ such that

$$
\begin{aligned}
& 0=m_{1} \circ m_{1}, \\
& 0=m_{2}\left(m_{1} \otimes|\mathbb{I}|\right)+m_{2}\left(\mathbb{I} \otimes \mu_{1}\right)-m_{1}\left(m_{2}\right), \\
& 0=m_{2}\left(m_{2} \otimes \mathbb{I}\right)-m_{2}\left(\mathbb{I} \otimes \mu_{2}\right) .
\end{aligned}
$$

A right module as above is a special case of the $A_{\infty}$-modules found in [8] (when using the sign conventions in this paper).

Definition 5.4 [8] A right $A_{\infty}$-module $M$ over an $A_{\infty}$-algebra $A$ is a set of maps $\left\{m_{i}\right\}_{i \in \mathbb{N}}$ with $m_{i}: M \otimes A^{\otimes(i-1)} \rightarrow M[i-2]$, and satisfying the following relations for each $n \geq 1$ :

$$
\begin{aligned}
& 0=\sum_{i+j=n+1}(-1)^{j(i+1)} m_{i}\left(m_{j} \otimes|\mathbb{I}|^{j \otimes(i-1)}\right) \\
& \quad+\sum_{i+j=n+1, k>0}(-1)^{k(j+1)+j(i+1)} m_{i}\left(\mathbb{I}^{\otimes k} \otimes \mu_{j} \otimes|\mathbb{I}|^{j \otimes(i-k-1)}\right) .
\end{aligned}
$$

$M$ is said to be strictly unital if for any $\xi \in M, m_{2}\left(\xi \otimes 1_{A}\right)=\xi$, but for $n>1$, $m_{n}\left(\xi \otimes a_{1} \otimes a_{2} \otimes \cdots \otimes a_{n-1}\right)=0$ if any $a_{i}=1_{A}$.

Our right modules correspond to $m_{i}=0$ for $i \geq 2$. Nevertheless, we will think of these as objects in the category of right $A_{\infty}$-modules. The morphisms in this category are the following.

Definition 5.5 [8] An $A_{\infty}$-morphism $\Psi$ of right $A$-modules $M$ and $M^{\prime}$ is a set of maps $\psi_{i}: M \otimes A^{\otimes(i-1)} \rightarrow M^{\prime}[i-1]$ for $i \in \mathbb{N}$, satisfying

$$
\begin{aligned}
& \sum_{i+j=n+1}(-1)^{(i+1)(j+1)} m_{i}^{\prime}\left(\psi_{j} \otimes|\mathbb{I}|^{(j+1) \otimes(i-1)}\right) \\
& =\sum_{i+j=n+1}(-1)^{j(i+1)} \psi_{i}\left(m_{j} \otimes|\mathbb{I}|^{j \otimes(i-1)}\right) \\
& \quad+\sum_{i+j=n+1, k>0}(-1)^{j(i+1)+k(j+1)} \psi_{i}\left(\mathbb{I}^{\otimes k} \otimes \mu_{j} \otimes|\mathbb{I}|^{j \otimes(i-k-1)}\right) .
\end{aligned}
$$

$\Psi$ is strictly unital if $\psi_{i}\left(\xi \otimes a_{1} \otimes \cdots \otimes a_{i-1}\right)=0$ when $a_{j}=1_{A}$ for some $j$ and $i>1$. The identity morphism $I_{M}$ is the collection of maps $i_{1}(\xi)=\xi, i_{j}=0$ for $j>1$. 
Definition 5.6 [8] Let $\Psi$ be an $A_{\infty}$-morphism from $M$ to $M^{\prime}$, and let $\Phi$ be an $A_{\infty}$-morphism from $M^{\prime}$ to $M^{\prime \prime}$. The composition $\Phi * \Psi$ is the morphism whose component maps for $n \geq 1$ are

$$
(\Phi * \Psi)_{n}=\sum_{i+j=n+1}(-1)^{(i+1)(j+1)} \phi_{i}\left(\psi_{j} \otimes|\mathbb{I}|^{(j+1) \otimes(i-1)}\right) .
$$

Definition 5.7 [8] Let $\Psi, \Phi$ be $A_{\infty}$-morphisms from $M$ to $M^{\prime} . \Psi$ and $\Phi$ are homotopic if there is a set of maps $\left\{h_{i}\right\}$ with $h_{i}: M \otimes A^{\otimes(i-1)} \rightarrow M^{\prime}[i]$ such that

$$
\begin{aligned}
\psi_{i}-\phi_{i}= & \sum_{i+j=n+1}(-1)^{(i+1) j} m_{i}^{\prime}\left(h_{j} \otimes|\mathbb{I}|^{j \otimes(i-1)}\right) \\
& +\sum_{i+j=n+1}(-1)^{(i+1) j} h_{i}\left(m_{j} \otimes|\mathbb{I}|^{j \otimes(i-1)}\right) \\
& +\sum_{i+j=n+1, k>0}(-1)^{k(j+1)+j(i+1)} h_{i}\left(\mathbb{I}^{\otimes k} \otimes \mu_{j} \otimes|\mathbb{I}|^{j \otimes(i-k-1)}\right)
\end{aligned}
$$

and for $i>1, h_{i}\left(\xi \otimes a_{1} \otimes \cdots \otimes a_{i-1}\right)=0$ when $a_{j}=1_{A}$ for some $j$.

The sign convention used in the previous definitions is that of Keller [5] with the Koszul sign rule

$$
(f \otimes g)(x \otimes y)=(-1)^{|f||y|}(f(x) \otimes g(y)) .
$$

Thus, as can be checked directly, the composition of morphisms is a morphism for this sign convention, and homotopy of morphisms is an equivalence relation (or see the Appendix). With these definitions, we are equipped to consider right $A_{\infty}$-modules up to homotopy equivalence. The following is our version of a standard result in the study of $A_{\infty}$-modules.

Proposition 5.8 Let $\left(M,\left\{m_{i}\right\}\right)$ be a strictly unital, right $A_{\infty}$-module over $\left(A,\left\{\mu_{i}\right\}\right)$, and let $\left(\bar{M}, \bar{m}_{1}\right)$ be a chain complex. Suppose there exist chain maps $\iota:\left(\bar{M}, \bar{m}_{1}\right) \rightarrow$ $\left(M, m_{1}\right)$ and $\pi:\left(M, m_{1}\right) \rightarrow\left(\bar{M}, \bar{m}_{1}\right)$, and a map $H: M \rightarrow M[1]$ satisfying the following relations:

$$
\begin{aligned}
\pi \circ \iota & =\mathbb{I}_{\bar{M}}, \\
\iota \circ \pi-\mathbb{I}_{M} & =m_{1} \circ H+H \circ m_{1}, \\
H \circ \iota & =0, \\
\pi \circ H & =0, \\
H^{2} & =0 .
\end{aligned}
$$


Then there are maps $\bar{m}_{i}: \bar{M} \otimes A^{\otimes(i-1)} \rightarrow \bar{M}$ for $i \geq 2$ such that $\left\{\bar{m}_{i}\right\}_{i=1}^{\infty}$ defines a strictly unital right $A_{\infty}$-module structure on $\bar{M}$. This structure is homotopy equivalent to $\left(M,\left\{m_{i}\right\}\right)$ through strictly unital morphisms which extend $\pi$ and $\iota$.

The proof supplies an explicit formula for computing $\bar{m}_{i}$ and the morphisms in the homotopy equivalence. First, we introduce some notation to simplify the formulas.

Definition 5.9 For positive integers $i_{1}, \ldots, i_{k}$, let

$$
N\left(i_{1}, \ldots, i_{k}\right)=\sum_{j}\left(i_{j}-1\right) \quad \text { and } \quad \alpha\left(i_{1}, \ldots, i_{k}\right)=\sum_{1 \leq r<s \leq k}\left(i_{r}-1\right)\left(i_{s}-1\right) .
$$

Definition 5.10 Let $i_{j} \geq 2$ be integers for $j=1, \ldots, k$. By $\left[i_{1}, \ldots, i_{k}\right]$ we will mean the composition

$$
\left(m_{i_{1}}\right)\left(H \otimes|\mathbb{I}|^{\otimes\left(i_{1}-1\right)}\right)\left(m_{i_{2}} \otimes|\mathbb{I}|^{i_{2} \otimes\left(i_{1}-1\right)}\right) \cdots\left(H \otimes|\mathbb{I}|^{\otimes\left(I-i_{k}\right)}\right)\left(m_{i_{k}} \otimes|\mathbb{I}|^{i_{k} \otimes\left(I-i_{k}\right)}\right),
$$

where we alternate between applying $m_{i_{j}}$ to the first $i_{j}$ entries in the tensor product and applying $H$ to the first factor in the tensor product.

Using this notation, we can define the action, morphisms, and homotopy. First, for $n \geq 2$ define a map $M \otimes A^{\otimes(n-1)} \rightarrow M[n-2]$ by

$$
\Sigma_{n}=\sum_{\substack{N\left(i_{1}, i_{2}, \ldots, i_{k}\right)=n-1 \\ i_{j} \geq 2}}(-1)^{\alpha\left(i_{1}, \ldots, i_{k}\right)}\left[i_{1}, \ldots, i_{k}\right] .
$$

We use $\Sigma_{n}$ to define $\bar{m}_{n}$ for $n \geq 1$ :

$$
\bar{m}_{n}:=\pi \circ \Sigma_{n} \circ\left(\iota \otimes \mathbb{I}^{\otimes(n-1)}\right) .
$$

For $n=1$ we use the boundary map $\bar{m}_{1}$. Then $\left\{\bar{m}_{i}\right\}_{i=1}^{\infty}$ equips $\bar{M}$ with the structure of a right $A_{\infty}$-module.

The morphisms which induce the homotopy equivalence are similarly defined. For $n=1$ we will use $\pi_{1}=\pi$ and $\omega_{1}=\iota$, while for $n>1$ we use

$$
\begin{aligned}
& \pi_{n}:=(-1)^{n}\left(\pi \circ \Sigma_{n} \circ\left(H \otimes|\mathbb{I}|^{\otimes(n-1)}\right)\right), \\
& \omega_{n}:=H \circ \Sigma_{n} \circ\left(\iota \otimes \mathbb{I}^{\otimes(n-1)}\right) .
\end{aligned}
$$

The additional $H$ means that these are maps $\pi_{n}: M \otimes \mathbb{I}^{\otimes(n-1)} \rightarrow \bar{M}[n-1]$ and $\omega_{n}: \bar{M} \otimes \mathbb{I}^{\otimes(n-1)} \rightarrow M[n-1]$. As defined, these morphisms satisfy the relations $\Pi * \Omega=I_{\bar{M}}$ and $\Omega * \Pi \simeq \Lambda I_{M}$, where $\lambda_{1}=H$,

$$
\lambda_{n}:=(-1)^{n}\left(H \circ \Sigma_{n} \circ\left(H \otimes|\mathbb{I}|^{\otimes(n-1)}\right)\right),
$$

and all homotopy equivalences occur in the category of (right) $A_{\infty}$-modules. 
We note that even when $m_{i} \equiv 0$ for $i>2$, a homotopy equivalence as described in Proposition 5.8 can have higher order action terms. Indeed, the new module structure is given by

$$
\bar{m}_{n}=(-1)^{\epsilon} \pi[2,2, \ldots, 2]\left(\iota \otimes \mathbb{I}^{n}\right),
$$

where there are exactly $n-12 \mathrm{~s}$ inside the square brackets and $\epsilon=0$ if $n \equiv 1,2$ modulo 4 , and $\epsilon=1$ if $n \equiv 3,4$ modulo 4 . Thus, in all cases

$$
\bar{m}_{2}=\pi \circ m_{2} \circ(\iota \otimes \mathbb{I}),
$$

which comes from appropriately adjusting $m_{2}$. The effect of $\pi$, however, is substantial when doing calculations. With this observation and Proposition 5.8, we can, by directly analyzing the diagrams before and after a Reidemeister move, see that the $A_{\infty}$-module structure is preserved up to homotopy equivalence. This affords us the difficult part of the next theorem.

Theorem 5.11 Let $\overleftarrow{\mathcal{T}}$ be an inside tangle with boundary $P_{2 n}$

(1) Let $\overleftarrow{T}$ be a diagram for $\overleftarrow{\mathcal{T}}$ in $\overleftarrow{\mathbb{H}}$. If $\mathfrak{o}_{1}$ and $\mathfrak{o}_{2}$ are two orderings of $\operatorname{CR}(\overleftarrow{T})$, then $\left\langle\left\langle T, \mathfrak{o}_{1} \rrbracket\right.\right.$ and $\left\langle\left\langle T, \mathfrak{o}_{2} \rrbracket\right.\right.$ are isomorphic type $A$ structures.

(2) If $\overleftarrow{T}_{1}$ and $\overleftarrow{T_{2}}$ are two diagrams for $\overleftarrow{\mathcal{T}}$, then $\left\langle\overleftarrow{T_{1}} \rrbracket\right.$ and $\left\langle\overleftarrow{T}_{2} \rrbracket\right.$ are homotopy equivalent type $A$ structure.

Corollary 5.12 The homotopy type of the type $A$ structure $\langle\overleftarrow{T} \rrbracket$, for any diagram $T$ of an inside tangle $\stackrel{\leftarrow}{\mathcal{T}}$, is a tangle invariant.

We will not prove these theorems here, as the proofs are modifications of those for the type $D$ structure for an outside tangle found in [9]. In addition, there are easier ways to prove these results once we have generalized the gluing theory in Section 7. Instead we content ourselves with computing some examples using Proposition 5.8 which will illustrate the argument.

How we will use this Suppose we have a chain complex $\left\{C_{i} \mid i \in \mathbb{Z}\right\}$ with explicit generators for each free chain group $C_{i}$. If the generators of $C_{i}$ are $\left\{x_{1}, \ldots, x_{n}\right\}$ and those for $C_{i-1}$ are $\left\{y_{1}, \ldots, y_{m}\right\}$ we can find a homotopy $H$ as in Proposition 5.8 by searching through the images $\partial x_{i}=\sum a_{i}^{j} y_{j}$ to find one where $a_{j}^{i}=u$ is a unit, for some $j$. We will reorder the generators so that this occurs for $i=j=1$. We can then construct a new chain complex where the other chain groups and boundary maps are taken to be the same, but $C_{i}^{\prime}$ is spanned by $x_{2}^{\prime}, \ldots, x_{n}^{\prime}$ and $C_{i-1}^{\prime}$ is spanned by $y_{2}^{\prime}, \ldots, y_{m}^{\prime}$. We let $\pi\left(x_{i}\right)=x_{i}^{\prime}$ for $i>1$ and $\pi\left(x_{1}\right)=0$, and likewise for the $y_{j}$. Otherwise $\pi$ is the identity. The new boundary $\partial_{i}^{\prime}: C_{i^{\prime}} \rightarrow C_{i-1}^{\prime}$ is given by 
$\partial^{\prime} x_{i}^{\prime}=(\pi \circ \partial)\left(x_{i}-a_{i}^{1} u^{-1} x_{1}\right)$. If we let $\iota\left(x_{i}^{\prime}\right)=x_{i}-a_{i}^{1} u^{-1} x_{1}$ and $H\left(y_{1}\right)=-u^{-1} x_{1}$ $(H(\beta)=0$ otherwise), then we are in the situation envisioned in Proposition 5.8. The map $\pi$ is the quotient map found by quotienting out the subcomplex generated by $\left\{x_{1}, \partial x_{1}\right\}$. The formulas involving $\pi$ are a specific presentation of this quotient complex for the specific basis. $\partial^{\prime}$ is computed by calculating $\pi \circ \partial$ in this presentation.

We now compute $\bar{m}_{2}\left(x_{i}^{\prime} \otimes e\right)$. Since $\bar{m}_{2}=\pi \circ m_{2} \circ(\iota \otimes \mathbb{I})$ we first compute

$$
m_{2}\left(\left(x_{i}-a_{i}^{1} u^{-1} x_{1}\right) \otimes e\right)=m_{2}\left(x_{i} \otimes e\right)-a_{i}^{1} u^{-1} m_{2}\left(x_{1} \otimes e\right),
$$

then compute $\pi$. In particular, suppose $\left\langle\partial x_{j}, y_{1}\right\rangle=a_{j}^{1}=0$ but $m_{2}\left(x_{j} \otimes e\right)=a y_{1}+Y$. Then $\bar{m}_{2}\left(x_{j}^{\prime} \otimes e\right)=\pi\left(a y_{1}+Y\right)=a\left(u^{-1} \sum_{j>1} a_{1}^{j} y_{j}\right)+Y$. This is the same process as for adjusting the boundary maps above.

However, suppose $\left\langle\partial x_{j}, y_{1}\right\rangle=a_{j}^{1} \neq 0$, but $m_{2}\left(x_{j} \otimes e_{1}\right)=a y_{1}+Y$ and $m_{2}\left(x_{1} \otimes e_{2}\right)=$ $W$. Then $\bar{m}_{3}\left(x_{j}^{\prime} \otimes e_{1} \otimes e_{2}\right)=\pi\left(m_{3}\left(x_{j} \otimes e_{1} \otimes e_{2}\right)\right)-\pi\left(m_{2}\left(H \circ m_{2}\left(x_{j} \otimes e_{1}\right) \otimes e_{2}\right)\right)$. We concentrate upon $\pi\left(m_{2}\left(H \circ m_{2}\left(x_{j} \otimes e_{1}\right) \otimes e_{2}\right)\right)=\pi\left(m_{2}\left(H\left(a y_{1}+Y\right) \otimes e_{2}\right)\right)$ $=\pi\left(m_{2}\left(-u^{-1} a x_{1} \otimes e_{2}\right)\right)=-u^{-1} a \cdot W$. We can thus pick up a higher order action.

\section{Examples of the type $A$ structure}

Example I (Reidemeister tangles) The three tangles below appear in the local description of the Reidemeister moves; we will analyze each in turn:
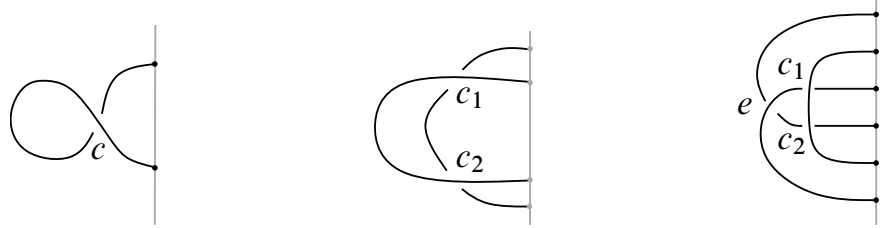

(a) First move For the RI move we have a tangle diagram $R_{I}$, over $P_{2}$ with one crossing. There are two resolutions, corresponding to $\rho=0$ and $\rho=1$, and the unique matching on $P_{2}$. Since the crossing is right handed, the 0 resolution has a single free circle. Writing the decoration on the cleaved circle first, we can think of the decorated resolutions as $z_{++}, z_{+-}, z_{-+}$, and $z_{--}$which occur in bigrading $\left(0, \frac{5}{2}\right),\left(0, \frac{1}{2}\right)$, $\left(0, \frac{1}{2}\right)$ and $\left(0,-\frac{1}{2}\right)$. For the 1 -resolution there is only the cleaved circle, so we get two state $t_{+}$in grading $\left(1, \frac{5}{2}\right)$ and $t_{-}$in $\left(1, \frac{3}{2}\right)$. We can compute $d_{\mathrm{APS}}$ as $d_{\mathrm{APS}}\left(z_{++}\right)=t_{+}$ and $d_{\mathrm{APS}}\left(z_{-+}\right)=t_{-} \cdot \mathcal{B} \Gamma_{1}$ has two non-idempotent elements $\overleftarrow{e_{C}}$ and $\overrightarrow{e_{C}}$. The actions of these elements are $z_{+*} \overrightarrow{e_{C}}=z_{-*}$ and $t_{+} \overrightarrow{e_{C}}=t_{-}$, since $\vec{e}_{C}$ only changes the sign on the cleaved circle. On the other hand, $z_{+-} \overleftarrow{e_{C}}=t_{-}$is the only non-trivial action for $\overleftarrow{e}_{C}$. Since $t_{+}$is not in the image of the action, or $d_{\text {APS }}$ except for $z_{++}$we can cancel 
both to result in the homotopy equivalent structure with generators $z_{-+}, z_{+-}, z_{--}$ and $t_{-}$.

We now cancel $t_{-}$through the image of $z_{-+}$. To compute the new action on $z_{+-}$ and $z_{--}$we consider $\iota$, which in this case is just inclusion, followed by $m_{2}\left(z_{*-} \otimes e\right)$ followed by projection. For $z_{+-}$there is the non-trivial action $m_{2}\left(z_{+-} \otimes \overrightarrow{e_{C}}\right)=z_{--}$, which projects to an action as well. However, while $m_{2}\left(z_{+-} \otimes \overleftrightarrow{e_{C}}\right)=t_{-}$, the projection will kill this image. Furthermore, all the higher actions vanish since $m_{2}$ acts trivially on $z_{--}$, and any computation of $\bar{m}_{n}$ for $n>2$ starts with $H \circ m_{2}\left(z_{+-} \otimes \overleftarrow{e_{C}}\right)=z_{-+}$, but the action on $z_{-+}$is trivial for all non-idempotents. The idempotent will fix $z_{-+}$, but this will be killed under $\pi$, or $H$, and the computation cannot proceed. Thus, $\left\langle\left\langle R_{I} \rrbracket\right.\right.$ is isomorphic to $\alpha_{+}=z_{+-}$in grading $\left(0, \frac{1}{2}\right)$ and $\alpha_{-}=z_{--}$in $\left(0,-\frac{1}{2}\right)$ with $d_{\mathrm{APS}} \equiv 0$ and the only non-trivial action term being $\alpha_{+} \cdot \overrightarrow{e_{C}}=\alpha_{-}$. This is isomorphic to $\left\langle U_{2} \rrbracket\right.$, where $U_{2}$ is the planar matching on $P_{2}$ found from untwisting the crossing.

(b) Second move For the RII move we analyze the tangle below, $R_{I I}$ over $P_{4}$, with two opposite crossings:

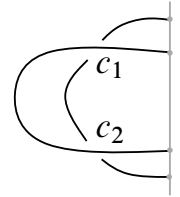

Thus $n_{+}=1$ and $n_{-}=1$ for every choice of orientation. We label the crossings from top to bottom. Now consider the states corresponding to the $01-$ resolution. There is a free circle in this resolution, and we can divide the states into $S_{01}^{+}$and $S_{01}^{-}$based on the decoration of the circle (we do this regardless of the matching $\vec{m}$ used to construct the state). $d_{\mathrm{APS}}$ maps $S_{01}^{+}$isomorphically to $S_{11}$ and $S_{00}$ isomorphically to $S_{01}^{-}$. The action $m_{2}(\xi \otimes e)$ for $\xi$ in $S_{01}^{+}$has image in $S_{01}^{+}$since it will not change the decoration on the + free circle, and merging the + free circle does not change the boundary of the state. Consequently if we cancel along the isomorphism from $S_{01}^{+}$to $S_{11}$ the image of $H$ is in $S_{01}^{+}$and the image of $\iota$ on $v \in S_{10}$ is a sum $v+v^{\prime}$, where $v^{\prime} \in S_{01}^{+}$. Thus $\pi \circ m_{2} \circ(\iota \otimes \mathbb{I})$ will have image equal to the part of $m_{2}(v \otimes e)$ in $S_{10}$, since $\pi$ will kill $S_{01}^{+}$. The only element $e$ for which the image may not be in $S_{10}$ is $\overleftarrow{e_{\gamma}}$ for the unique class of bridges $\gamma$ in the boundary of any element in $S_{10}$. Its action would have image in $S_{11}$, but does not contribute to $\underline{m}_{n}$ for $n>1$ since $H: S_{11} \rightarrow S_{01}^{+}$, and thus any additional actions stay in $S_{01}^{+}$, which will be killed by $\pi$.

The effect of the cancellation, therefore, is to reduce our module to $S_{10} \oplus S_{00} \oplus S_{01}^{-}$ with action defined by restricting the image of $m_{2}$ to the remaining summands. Now a similar argument applies to the isomorphism found by the image $d_{\mathrm{APS}} \mid S_{00}$ in $S_{01}^{-}$. Now, however, no element from $S_{10}$ can have a term in its action or boundary within $S_{01}$, so 
these will be unchanged. After the cancellation we obtain all the states in $S_{10}$ having trivialized the $\overleftarrow{\gamma}$ action, but otherwise left the action unchanged. This is the same type $A$ structure as for the matching of the top point in $P_{4}$ with the bottom, and the second with the third. Thus it is isomorphic to the structure obtained after removing the crossings with the RII move. Being in $S_{10}$ means the states have no free circle, and just receive grading based on the cleaved circles. Furthermore, they are shifted by $(1,1)+(-1,1-2 \cdot 1)=(0,0)$ when we account for the resolution and the crossings. Thus, as a bigraded type $A$ structure the RII tangle is homotopy equivalent to the planar matching obtained from the RII move.

(c) Third move (sketch) Let $R_{b}$ be the diagram before the move and $R_{a}$ be the diagram after:
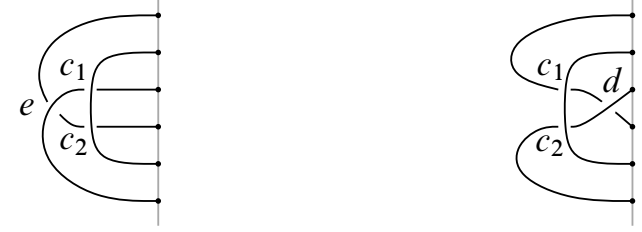

In each, if we 0 -resolve the second crossing from the top we obtain a diagram with an RII move. As usual the states with a 1 -resolution here give rise to the same type $A$ structure. It is enough then to see what happens in the 0 -resolved sub-module. As with the RII move we can use $d_{\mathrm{APS}}$ to leave $S_{100}$ with its action intact, including the action of $e_{\gamma_{1}}^{\leftarrow}$ which has image in $S_{110}$. However, $d_{\text {APS }}$ now maps $S_{001}^{+}$to $S_{101} \oplus S_{011}$, isomorphically to each factor, given by minus the Khovanov maps. We let $\xi$ be the state in $S_{101}$ then $\xi^{\prime}$ is the corresponding state, found by planar isotopy, in $S_{011}$. The effect of $\pi$ is to identify $\xi$ with $-\xi^{\prime}$. Now, let $v$ be a state in $S_{100}$ and let $v^{\prime}=H \circ d_{\text {APS }}(v)$ in $S_{001}^{+}$. Then $\iota(v)=v+v^{\prime}$. The action $m_{2}\left(\left(v+v^{\prime}\right) \otimes \overleftrightarrow{e_{\gamma_{2}}}\right)=m_{2}\left(v \otimes \overleftrightarrow{e_{\gamma_{2}}}\right)$ since the action of $e_{\gamma_{2}}^{\overleftarrow{ }}$ on $S_{001}^{+}$is trivial ( $\gamma_{2}$ is used in the calculation of $d_{\mathrm{APS}}$ for these states). If $m_{2}\left(v \otimes e_{\gamma_{2}}\right)$ is non-zero in $S_{101}$, then the effect of $\pi$ is to identify it with

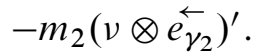

If we repeat this argument with $R_{a}$, with the same crossing ordering, we get $S_{001}$ being the planar matching diagram and $S_{100}^{+}$being used in the cancellation process.

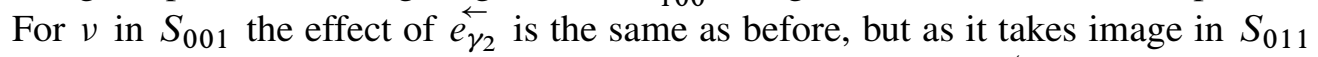
it occurs with a minus sign. On the other hand, the image of $\overleftarrow{e_{\gamma_{1}}}$ will be in $S_{101}$, occurring with a minus sign, due to the crossing ordering, and thus will be identified with $-(-\eta)$, where $\eta$ is the image $m_{2}\left(v \otimes \overleftarrow{e_{\gamma_{1}}}\right)$ from $R_{b}$ in the previous paragraph. As such the actions of the bridges will be the same, and the APS-complexes will be the same. It is straightforward to see that the higher actions all vanish. 
Example II (Hopf tangle) For the Hopf tangle over $P_{2}$

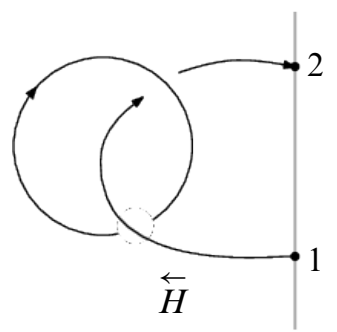

we will enumerate the crossings from the top of the page to the bottom, and write states with the decoration of the cleaved circle first. For the moment we will ignore orientations. There are four states $s_{ \pm, \pm}^{00}$ in homological grading 0 and quantum gradings $\pm \frac{1}{2} \pm 1$. There are two states $s_{ \pm}^{10}$ for the 10-resolution, and two states $s_{ \pm}^{01}$ for the 01 . These occur in the bigradings $\left(1,1 \pm \frac{1}{2}\right)$. Finally, there are four states $s_{ \pm \pm}^{11}$ with bigrading $\left(2,2 \pm 1 \pm \frac{1}{2}\right)$. For these states:

(1) $d_{\mathrm{APS}}$ is computed as

$$
\begin{aligned}
s_{++}^{00} & \rightarrow s_{+}^{10}+s_{+}^{01}, & s_{-+}^{00} \rightarrow s_{-}^{10}+s_{-}^{01}, & s_{+}^{10} \rightarrow s_{+-}^{11}, \\
s_{-}^{10} & \rightarrow s_{--}^{10}, & s_{+}^{01} \rightarrow-s_{+-}^{11}, & s_{-}^{01} \rightarrow-s_{--}^{11} .
\end{aligned}
$$

(2) The action of $\overrightarrow{e_{C}}$ simply takes $s_{+*}^{*} \rightarrow s_{-*}^{*}$, where $*$ matches anything in those spots.

(3) The action of $\overleftarrow{e_{C}}$ is given by

$$
s_{+-}^{00} \rightarrow s_{-}^{10}+s_{-}^{01}, \quad s_{+}^{10} \rightarrow s_{-+}^{11}, \quad s_{+}^{01} \rightarrow-s_{-+}^{11} .
$$

If we cancel $s_{++}^{00}$ with $s_{+}^{10}$ we will have no effect except to remove these generators, as $s_{+}^{10}$ does not occur in the image of $d_{\text {APS }}$ or the action for any other state. Once we have done that, we can cancel $s_{+}^{01}$ with $-s_{+-}^{11}$ with no other effect, since $s_{+-}^{11}$ only occurs in the image of a previously canceled state. $s_{--}^{11}$ will then appear only in the image $d_{\mathrm{APS}}\left(s_{-}^{01}\right)$ and $d_{\mathrm{APS}}\left(s_{-}^{10}\right)$ (since $s_{+-}^{11}$ has been canceled, otherwise we would also need to include it in the image of $\left.\overrightarrow{e_{C}}\right)$. As $d_{\mathrm{APS}}\left(s_{-}^{01}\right)=-s_{--}^{11}$, we can cancel these without affecting the rest of the maps. Finally we can cancel $s_{-+}^{00}$ with $s_{-}^{10}$. Now, $s_{-}^{10}$ occurs as $s_{+-}^{00} \overleftarrow{e_{C}}$, but there are no other terms to consider, so the effect of the cancellation (through the projection $\pi$ ) is to cancel this portion of the action of $\overleftarrow{e}_{C}$.

Following these steps results in $s_{+-}^{00}$ and $s_{--}^{00}$ in bigrading $\left(0,-\frac{1}{2}\right)$ and $\left(0,-\frac{3}{2}\right)$, and $s_{++}^{11}$ in bigrading $\left(2, \frac{7}{2}\right)$ and $s^{-+}$in bigrading $\left(2, \frac{5}{2}\right)$. The residual action is that of $\overrightarrow{e_{C}}$, which takes $s_{+-}^{00}$ to $s_{--}^{00}$ and $s_{++}^{11}$ to $s_{-+}^{11}$. 
The Hopf tangle will either have two positive or two negative crossings, depending upon the orientation of the components. If there are two positive crossings we will shift the bigrading up $(0,2)$. Otherwise, for negative crossings, we add $(-2,-4)$ to each bigrading.

Consequently, for the positive Hopf tangles we will have

$$
\mathbb{F}_{(0,3 / 2)} \stackrel{\overrightarrow{e_{C}}}{\longrightarrow} \mathbb{F}_{(0,1 / 2)} \quad \text { and } \quad \mathbb{F}_{(2,11 / 2)} \stackrel{\overrightarrow{e_{C}}}{\longrightarrow} \mathbb{F}_{(2,9 / 2)}
$$

For negative Hopf tangles we will have

$$
\mathbb{F}_{(-2,-9 / 2)} \stackrel{\overrightarrow{e_{C}}}{\longrightarrow} \mathbb{F}_{(-2,-11 / 2)} \quad \text { and } \quad \mathbb{F}_{(0,-1 / 2)} \stackrel{\overrightarrow{e_{C}}}{\longrightarrow} \mathbb{F}_{(0,-3 / 2)}
$$

\section{Gluing inside and outside tangles}

Let $\overleftarrow{\mathcal{T}_{1}}$ be an inside tangle for $P_{2 n}$ and $\overrightarrow{\mathcal{T}_{2}}$ be an outside tangle. We let $\mathcal{T}=\overleftarrow{\mathcal{T}_{1}} \# \overrightarrow{\mathcal{T}_{2}}$ be the link in $\mathbb{R}^{3}$ obtained by gluing $\mathbb{R}^{3}$ to $\overrightarrow{\mathbb{R}}^{3}$ and thereby gluing $\overleftarrow{\mathcal{T}}_{1}$ to $\overrightarrow{\mathcal{T}}_{2}$ along $P_{2 n}$. Likewise, if $\overleftarrow{T_{1}}$ is a diagram for $\overleftarrow{\mathcal{T}_{1}}$ in $\overleftarrow{\mathbb{H}}$ and $\vec{T}_{2}$ is a diagram for $\overrightarrow{\mathcal{T}}_{2}$ in $\overrightarrow{\mathbb{H}}$, we can glue these diagrams along $P_{2 n}$ to obtain a diagram $T$ for $\mathcal{T}$.

In [9], we showed how to associate a bigraded type $D$ structure to $\vec{T}_{2}$ whose homotopy type is an isotopy invariant of $\overrightarrow{\mathcal{T}}_{2}$. In particular, we constructed a bigrading preserving map

$$
\left.\left.\vec{\delta}_{T}: \llbracket \vec{T}\right\rangle \rightarrow \mathcal{B} \Gamma_{n} \otimes_{\mathcal{I}} \llbracket \vec{T}\right\rangle[(-1,0)]
$$

which satisfies the type $D$ structure equation

$$
\left(\mu_{\mathcal{B} \Gamma_{n}} \otimes \mathbb{I}\right)\left(\mathbb{I} \otimes \vec{\delta}_{T}\right) \vec{\delta}_{T}+\left(d_{\Gamma_{n}} \otimes|\mathbb{I}|\right) \vec{\delta}_{T}=0,
$$

where $\mu_{\mathcal{B} \Gamma_{n}}: \mathcal{B} \Gamma_{n} \otimes \mathcal{B} \Gamma_{n} \rightarrow \mathcal{B} \Gamma_{n}$ is the multiplication map on $\mathcal{B} \Gamma_{n}$.

Definition 7.1 By $\left\langle\left\langle T_{1} \rrbracket \otimes \llbracket T_{2}\right\rangle\right\rangle$ we mean the bigraded module

$$
\left\langle T_{1} \rrbracket \otimes_{\mathcal{I}_{n}} \llbracket T_{2}\right\rangle
$$

equipped with the map

$$
\partial^{\otimes}(x \otimes y)=d_{\mathrm{APS}}(x) \otimes|y|+\left(m_{2, T_{1}} \otimes \mathbb{I}\right)\left(x \otimes \vec{\delta}_{T_{2}}(y)\right) .
$$

Proposition 7.2 $\partial^{\bigotimes}$ is a $(1,0)$-differential map on $\left\langle\left\langle T_{1} \rrbracket \otimes \llbracket T_{2}\right\rangle\right.$. 
Proof First, we rewrite $\partial^{\bigotimes}$ as an operator:

$$
\partial^{\bigotimes}=d_{\mathrm{APS}} \otimes|\mathbb{I}|+\left(m_{2, T_{1}} \otimes \mathbb{I}\right)\left(\mathbb{I} \otimes \vec{\delta}_{T_{2}}\right) .
$$

We note that $\mathbb{I} \otimes \vec{\delta}_{T_{2}}$ is a $(1,0)-$ map $\left\langle\left\langle T_{1} \rrbracket \otimes_{\mathcal{I}} \llbracket T_{2}\right\rangle \rightarrow\left\langle\left\langle T_{1} \rrbracket \otimes_{\mathcal{I}} \mathcal{B} \Gamma_{n} \otimes_{\mathcal{I}} \llbracket T_{2}\right\rangle\right.\right.$, while $m_{2, T_{1}} \otimes \mathbb{I}$ preserves the bigrading as a map $\left\langle\left\langle T_{1} \rrbracket \otimes_{\mathcal{I}} \mathcal{B} \Gamma_{n} \otimes_{\mathcal{I}} \llbracket T_{2}\right\rangle \rightarrow\left\langle\left\langle T_{1} \rrbracket \otimes_{\mathcal{I}} \llbracket T_{2}\right\rangle\right\rangle\right.$. In addition, $d_{\text {APS }}$ is a $(1,0)$-map. Hence $\partial^{\bigotimes}$ is a $(1,0)$-map. We need to verify that $\partial^{\bigotimes}$ is a differential. This result follows from Section A.9 in the Appendix, and that when $m_{i, T_{1}}=0$ for $i>2, m_{1, T_{1}}=d_{\mathrm{APS}}$, implies that $\partial^{\bigotimes}$ above coincides with the definition in the Appendix.

By $\langle\langle T\rangle$ we will mean the usual bigraded Khovanov complex over $\mathbb{Z}$, equipped with its invariant bigrading.

Proposition 7.3 $\langle T\rangle \cong\left(\left\langle T_{1} \rrbracket \otimes \llbracket T_{2}\right\rangle, \partial^{\bigotimes}\right)$.

Important comment We have not required that the orientations on $T_{1}$ and $T_{2}$ match along $P_{n}$. If they do, $\langle\langle T\rangle$ is exactly the Khovanov complex from [6], as described in [3]. However, the statement still holds even if the orientations do not match. The Khovanov complex in the latter case is for a link with a finite number of orientation changes, constructed in the same manner as before. Now, however, it has an invariant bigrading only as long as isotopies do not take a strand across a point where the orientation changes. In the latter case there is a bigrading shift of $\pm(1,3)$ due to the conversion of a negative crossing to a positive crossing, or vice versa.

Proof We start by identifying the generators of $\left\langle\left\langle T_{1} \rrbracket \otimes_{\mathcal{I}} \llbracket T_{2}\right\rangle\right\rangle$ with the generators of $\langle T\rangle$. For $\left(r_{1}, s_{1}\right) \otimes_{\mathcal{I}}\left(r_{2}, s_{2}\right) \neq 0$ we need that $I_{\partial\left(r_{1}, s_{1}\right)} \cdot\left(r_{2}, s_{2}\right) \neq 0$ since $\left(r_{1}, s_{1}\right) \cdot$ $I_{\partial\left(r_{1}, s_{1}\right)}=\left(r_{1}, s_{1}\right)$. However, only $I_{\partial\left(r_{2}, s_{2}\right)} \cdot\left(r_{2}, s_{2}\right) \neq 0$, so $\underset{\rightarrow}{\rightarrow}\left(r_{1}, s_{1}\right)=\partial\left(s_{2}, r_{2}\right)=$ $(L, \sigma)$. If $r_{1}=\left(\rho_{1}, \vec{m}_{1}\right)$ and $r_{2}=\left(\vec{m}_{2}, \rho_{2}\right)$, we use $\vec{m}_{1}=\vec{L}$ to identify $\vec{m}_{1}$ with the $\operatorname{arcs}$ in $\rho_{2}\left(T_{2}\right)$, and likewise we can identify $\overleftarrow{m_{2}}=\overleftarrow{L}$ with the $\operatorname{arcs}$ in $\rho_{1}\left(T_{1}\right)$. Since $s_{1}$ and $s_{2}$ restrict to $\sigma$, we can decorate $\rho_{1}\left(T_{1}\right) \# \rho_{2}\left(T_{2}\right)$ by $s_{1} \# s_{2}$ to get a resolution diagram for $T$ where every circle is unambiguously decorated with \pm .

Furthermore, we can reverse the construction. If $\rho$ is a resolution of $T$, we let $\rho_{1}$ be $\rho$ restricted to those crossings in $\overleftarrow{\mathbb{H}} \cap T=T_{1}$ and $\rho_{2}$ be $\rho$ restricted to $\overrightarrow{\mathbb{H}} \cap T=T_{2}$. Furthermore, the arcs in $\rho_{2}\left(T_{2}\right)$ form an (outside) planar matching $\vec{m}_{1}$, and we define $r_{1}=\left(\rho_{1}, \vec{m}_{1}\right)$. Likewise the arcs of $\rho_{1}\left(T_{1}\right)$ define an (inside) planar matching $\overleftarrow{m_{2}}$ and we let $r_{2}=\left(\overleftarrow{m_{2}}, \rho_{2}\right)$. A generator of $\langle T T\rangle$ is a pair $(\rho, s)$, where $s$ is a decoration of $\operatorname{CIR}(\rho(T))$. By restriction $s$ defines decorations, $s_{1}, s_{2}$ on $r_{1}\left(T_{1}\right)$ and $r_{2}\left(T_{2}\right)$ with $\partial\left(r_{1}, s_{1}\right)=\partial\left(r_{2}, s_{2}\right)$. It is straightforward to see that $\left(r_{1}, s_{1}\right) \otimes_{\mathcal{I}}\left(r_{2}, s_{2}\right)=(\rho, s)$, so this is the inverse of the previous map. 
Furthermore, the bigrading of $\left(r_{1}, s_{1}\right) \otimes_{\mathcal{I}}\left(r_{2}, s_{2}\right)$ coming from the tensor product is identical to that of $(\rho, s)$ from the construction of $\left\langle\langle T\rangle\right.$. The bigrading of $\left(r_{1}, s_{1}\right) \otimes_{\mathcal{I}}$ $\left(r_{2}, s_{2}\right)$ is the sum

$$
\begin{aligned}
h\left(r_{1}\right)-n_{-} & \left(T_{1}\right), h\left(r_{1}\right)+q\left(r_{1}, s_{1}\right)+\frac{1}{2} \iota\left(\partial\left(r_{1}, s_{1}\right)\right)+n_{+}\left(T_{1}\right)-2 n_{-}\left(T_{1}\right) \\
& +h\left(r_{2}\right)-n_{-}\left(T_{2}\right), h\left(r_{2}\right)+q\left(r_{2}, s_{2}\right)+\frac{1}{2} \iota\left(\partial\left(r_{2}, s_{2}\right)\right)+n_{+}\left(T_{2}\right)-2 n_{-}\left(T_{2}\right) .
\end{aligned}
$$

However $h\left(r_{1}\right)+h\left(r_{2}\right)$ is the number of 1 -resolutions in $\rho_{1}\left(T_{1}\right)$ added to the number in $\rho_{2}\left(T_{2}\right)$, which equals the total number in $\rho(T)$. Likewise, since they are counts over crossings, $n_{+}\left(T_{1}\right)+n_{+}\left(T_{2}\right)=n_{+}(T)$ and $n_{-}\left(T_{1}\right)+n_{-}\left(T_{2}\right)=n_{-}(T)$. Finally, $\iota\left(\partial\left(r_{1}, s_{1}\right)\right)+\iota\left(\partial\left(r_{2}, s_{2}\right)\right)=2 \iota(L, \sigma)$ so the second entry in the bigrading equals the sum of the decorations on the free circles in $r_{1}\left(T_{1}\right)$ plus the sum of the decorations on the circles in $(L, \sigma)$ plus the sum of the decorations on the free circles in $r_{2}\left(T_{2}\right)$. In $\rho(T)$ this is just the quantum grading for the usual Khovanov generator. Thus the bigrading of $\left(r_{1}, s_{1}\right) \otimes_{\mathcal{I}}\left(r_{2}, s_{2}\right)$ is $\left(h(\rho)-n_{-}(T), h(\rho)+q(\rho, s)+n_{+}(T)-2 n_{-}(T)\right)$ which is the bigrading of $(r, s)$ in $\langle\langle T\rangle$. The tensor product identifies $\langle\langle T\rangle$ with $\left\langle T_{1} \rrbracket \otimes_{\mathcal{I}} \llbracket T_{2}\right\rangle$ as bigraded modules over $\mathbb{Z}$.

To see that $\partial^{\bigotimes}$ is the $(1,0)-$ Khovanov differential $\partial_{\mathrm{KH}}$ under this isomorphism, we must first specify the order of the crossings to be used in calculating the signs in $\partial_{\mathrm{KH}}$. The chain isomorphism type of $\langle\langle T\rangle\rangle$ is unaffected by this choice of ordering [6]. If $\mathfrak{o}_{i}$ is the ordering of the crossings in $T_{i}$ and, then $\mathfrak{o}_{1} \| \mathfrak{o}_{2}$ is an ordering of the crossings for $T$, which we now fix. In short, all the crossings of the inside tangle $T_{1}$ come before all the crossings of $T_{2}$, and in the same order as in $T_{1}$.

We compute $\partial^{\bigotimes}$ in stages. First $\left(d_{\mathrm{APS}} \otimes|\mathbb{I}|\right)\left[\left(r_{1}, s_{1}\right) \otimes_{\mathcal{I}}\left(r_{2}, s_{2}\right)\right]$ is a sum over the crossings of $T_{1}$. For each crossing $c$ we get either 0 or $\left.(-1)^{m}(-1)^{h\left(r_{2}\right.}\right)\left(r^{\prime}, s^{\prime}\right) \otimes_{\mathcal{I}}$ $\left(r_{2}, s_{2}\right)$, where $m$ is the number of 1 -resolutions in $\left(r_{1}, s_{1}\right)$ following $c, h\left(r_{2}\right)$ is the total number of 1 -resolutions in $r_{2}$, and $\left(r^{\prime}, s^{\prime}\right)$ is as specified previously, which has $\partial\left(r^{\prime}, s^{\prime}\right)=\partial\left(r_{1}, s_{1}\right)$. Consequently, $m+h\left(r_{2}\right)$ is the number of $1-$ resolutions of $T$ following $c$ in our fixed order, and $\left(r^{\prime}, s^{\prime}\right) \otimes_{\mathcal{I}}\left(r_{2}, s_{2}\right)$ is a generator of $\langle\langle T\rangle$. Following the definition of $d_{\mathrm{APS}}$ this is precisely a term in $\partial_{\mathrm{KH}}(r, s)$. In fact, the sum of these is precisely the terms in $\partial_{\mathrm{KH}}(r, s)$ which have the same decorated cleaved link, and occur from a crossing change in $T \cap \overleftarrow{\mathbb{H}}$. Those terms in $\partial_{\mathrm{KH}}(r, s)$ which have the same decorated cleaved link, and occur from a crossing change in $\overrightarrow{\mathbb{H}} \cap T$ correspond to terms in $\left(m_{2, T_{1}} \otimes \mathbb{I}\right)\left(\mathbb{I} \otimes \vec{\delta}_{T_{2}}\right)$ applied to $\left(r_{1}, s_{1}\right) \otimes_{\mathcal{I}}\left(r_{2}, s_{2}\right)$. In the definition of $\vec{\delta}_{T_{2}}\left(r_{2}, s_{2}\right)$ there is a term $I_{\partial\left(r_{2}, s_{2}\right)} \otimes d_{\mathrm{APS}}\left(r_{2}, s_{2}\right)$. Since $\partial\left(r_{2}, s_{2}\right)=\partial\left(r_{1}, s_{1}\right)$ we conclude that $\left(m_{2, T_{1}} \otimes \mathbb{I}\right)\left(\left(r_{1}, s_{1}\right) \otimes I_{\partial\left(r_{2}, s_{2}\right)} \otimes d_{\mathrm{APS}}\left(r_{2}, s_{2}\right)\right)=\left(r_{1}, s_{1} \otimes_{\mathcal{I}} d_{\mathrm{APS}}\left(r_{2}, s_{2}\right)\right.$. Note that the signs are also correct for $\partial_{\mathrm{KH}}$ since the sign of a term in $I_{\partial\left(r_{2}, s_{2}\right)} \otimes d_{\mathrm{APS}}\left(r_{2}, s_{2}\right)$ is $(-1)^{m}$, where $m$ is the number of 1 -resolutions in $r_{2}$ following the crossing that yields the term. As this crossing follows all those of $T_{1}$, the same sign is used in $\partial_{\mathrm{KH}}$. 
This leaves the terms of $\partial_{\mathrm{KH}}\left[\left(r_{1}, s_{1}\right) \otimes_{\mathcal{I}}\left(r_{2}, s_{2}\right)\right]$ which change the decorated, cleaved link. We divide them into two groups, based on whether the crossing change giving the term occurs in $\overleftarrow{\mathbb{H}}$ or $\overrightarrow{\mathbb{H}}$. We start with those in $\overrightarrow{\mathbb{H}}$. Each such crossing gives an active arc which is either in $\overrightarrow{\mathrm{BR}}\left(r_{2}\right)$ or $\operatorname{DEC}\left(r_{2}, s_{2}\right)$. In the first case, $\vec{\delta}_{T_{2}}\left(r_{2}, s_{2}\right)$ will have a term, or two terms, $(-1)^{m}\left(\vec{e}_{\gamma} \otimes\left(r^{\prime}, s^{\prime}\right)\right)$ which corresponds to the crossing change. As before, $m$ is the number of 1 -resolved crossings following the crossing. This is the same sign as in $\partial_{\mathrm{KH}}$, and the decorations on the decorated, cleaved link also follow the pattern for Khovanov homology.

In $\left(m_{2, T_{1}} \otimes \mathbb{I}\right)\left(\mathbb{I} \otimes \vec{\delta}_{T_{2}}\right)$ we get the term $(-1)^{m}\left(m_{2, T_{1}} \otimes \mathbb{I}\right)\left[\left(r_{1}, s_{1}\right) \otimes \vec{e}_{\gamma} \otimes\left(r^{\prime}, s^{\prime}\right)\right]$. From the definition of $m_{2, T_{1}}$ the action of $\vec{e}_{\gamma}$ on $\left\langle T_{1} \rrbracket\right.$ is just to change the decorated, cleaved link to have the same boundary as $\left(r^{\prime}, s^{\prime}\right)$ (which occurs purely in $\vec{m}_{1}$ ). Consequently, we obtain the tensor product of compatible pairs, and we replicate the term in $\partial_{\mathrm{KH}}$. The case of an $\operatorname{arc}$ in $\operatorname{DEC}\left(r_{2}, s_{2}\right)$ is similar, except only the decoration on one circle changes, and not the underlying cleaved link. This is the effect of $\overrightarrow{e_{C}}$, for that circle, on $\left\langle T_{1} \rrbracket\right.$.

This leaves the terms of $\partial_{\mathrm{KH}}$ which come from crossing changes in $\overleftarrow{\mathbb{H}}$ that change the decorated, cleaved link. Let $c$ be such a crossing, and $\gamma$ be the active arc. Suppose $\gamma$ has image in $\overleftarrow{\operatorname{BR}}\left(\partial\left(r_{1}, s_{1}\right)\right)$, which we will denote by $\gamma^{\prime}$. There is then a term in $\vec{\delta}_{T_{2}}\left(r_{2}, s_{2}\right)$ of the form $(-1)^{h\left(r_{2}\right)}\left(\overleftarrow{e_{\gamma^{\prime}}} \otimes\left(r_{2}^{\prime}, s_{2}^{\prime}\right)\right)$, where $\left(r_{2}^{\prime}, s_{2}^{\prime}\right)$ is the result of $\gamma^{\prime}$ surgery on $r\left(T_{2}\right) \cap \overleftarrow{\mathbb{H}}$ which reflects the decoration changes necessary for the Khovanov differential. In $(-1)^{h\left(r_{2}\right)}\left(m_{2, T_{1}} \otimes I\right)\left(\left(r_{1}, s_{1}\right) \otimes \overleftarrow{e_{\gamma^{\prime}}} \otimes\left(r_{2}^{\prime}, s_{2}^{\prime}\right)\right)$ we get a sum over all the terms in $\partial_{\mathrm{KH}}$ which correspond to $\gamma^{\prime}$ and the decoration changes for $\overleftarrow{e_{\gamma^{\prime}}}$, but with sign $(-1)^{h\left(r_{2}\right)}(-1)^{m}$, where $m$ is the number of 1 -resolved crossings following that for $\gamma\left(\right.$ not $\left.\gamma^{\prime}\right)$ in ordering on the crossings of $T_{1}$. However, $h\left(r_{2}\right)+m$ is the number of 1 -resolved crossings following that for $\gamma$ in the ordering on $T$. Thus, the sign is the same as that for $\partial_{\mathrm{KH}}$. If $\gamma \in \operatorname{DEC}\left(r_{1}, s_{1}\right)$ then the argument is the same except that the term in $\partial_{\mathrm{KH}}$ comes from the action of $(-1)^{h\left(r_{2}\right)}\left(\overleftarrow{e_{C}} \otimes\left(r_{2}, s_{2, C}\right)\right)$, where $C$ is the cleaved circle whose decoration changes. Note that a crossing change can occur in multiple terms, but that with the decoration changes included, each crossing and decoration change occurs in precisely one way above. Thus we recover all the terms of $\partial_{\mathrm{KH}}(r, s)$ with the correct signs from the ordering of crossings.

The advantage of using $\left\langle\left\langle T_{1} \rrbracket \otimes \llbracket T_{2}\right\rangle\right\rangle$ arises from the ability to separately simplify $\left\langle T_{1} \rrbracket\right.$ and $\left.\left.\llbracket T_{2}\right\rangle\right\rangle$ without changing the homotopy type of $\left\langle\left\langle T_{1} \rrbracket \otimes \llbracket T_{2}\right\rangle\right\rangle$. We show this in the Appendix through a series of propositions which replicate, for our sign conventions, results from [8]. In particular, Proposition A.43 and the corollary to A.45 imply the following result. 
Proposition 7.4 Suppose $(N, \delta)$ is homotopy equivalent, as a type $D$ structure over $\mathcal{B} \Gamma_{n}$, to $\left.\llbracket T_{2}\right\rangle$, and $\left(M,\left\{m_{i}\right\}\right)$ is homotopy equivalent to $\left\langle T_{1} \rrbracket\right.$, as a type $A$ structure. Then $\left(M,\left\{m_{i}\right\}\right) \otimes(N, \delta) \simeq\left\langle\left\langle T_{1} \rrbracket \otimes \llbracket T_{2}\right\rangle \simeq\left\langle\left\langle T_{1} \# T_{2}\right\rangle\right.\right.$.

In the preceding proposition, we assume that the homotopy equivalences preserve the quantum grading.

We have seen in Section 5 how to affect such a homotopy equivalence by simplifying the chain complex $\left(《 T_{1} \rrbracket, d_{\mathrm{APS}}\right)$. A similar result holds for the type $D$ structure on $\left\langle\left\langle T_{2} \rrbracket\right.\right.$ : simplifications of the chain complex with differential $d_{\text {APS }}$ results in a homotopy equivalent type $D$ structure on the simplified complex. Over a field, $\mathbb{F}$, such simplifications show that $\left(\left\langle T_{1} \rrbracket \otimes \mathbb{F}, d_{\mathrm{APS}}\right) \simeq H_{*, \mathbb{F}}\left(\left\langle T_{1} \rrbracket\right)\right.\right.$, where the homology is taken with respect to $d_{1}$ and similarly for $\left.\left.\left(\llbracket T_{2}\right\rangle\right\rangle \otimes \mathbb{F}, d_{\text {APS }}\right)$. These homologies are determined by the tangle homology of Asaeda, Przytycki, and Sikora. The following corollary is a consequence.

Corollary 7.5 There is a type $A$ structure on $H_{*, \mathbb{F}}\left(\left\langle T_{1} \rrbracket\right)\right.$ and a type $D$ structure on $\left.\left.H_{*, \mathbb{F}}\left(\llbracket T_{2}\right\rangle\right)\right)$ for which

$$
\langle T\rangle \simeq H_{*, \mathbb{F}}\left(\left\langle T_{1} \rrbracket\right) \otimes H_{*, \mathbb{F}}\left(\llbracket T_{2}\right\rangle\right) .
$$

For example, this result applies to the rational coefficient theory and the theory over $\mathbb{Z} / 2 \mathbb{Z}$.

\section{Examples of pairing type $A$ and type $D$ structures}

Example I (Reidemeister invariance of Khovanov homology) Suppose that $L$ and $L^{\prime}$ are two link diagrams for an oriented link in $S^{3}$. Furthermore, suppose they differ by a Reidemeister move. If $D^{2} \subset \mathbb{R}^{2}$ is the local region in which the move occurs, we can use $\partial D^{2}$ and the orientation on $\mathbb{R}^{2}$ to think of $R=L \cap D^{2}$ as the inside tangle, and $\vec{L}=L \cap\left(\mathbb{R}^{2} \backslash D\right)$ as the outside tangle. Then $\langle\langle L\rangle \cong\langle R \rrbracket \otimes \llbracket \vec{L}\rangle$. If we let $R^{\prime}=L^{\prime} \cap D^{2}$ then $\left\langle\left\langle L^{\prime}\right\rangle \rrbracket \cong\left\langle R^{\prime} \rrbracket \otimes \llbracket \vec{L}\right\rangle\right.$. In Section 6 we computed the type $A$ structure for three of the tangles involved in the Reidemeister moves. In each, we saw that the type $A$ structure was homotopy equivalent to the structure obtained for the tangle after applying the Reidemeister move. Due to the results in Section A.9, this implies that

$$
\langle L\rangle \cong\left\langle\langle R \rrbracket \otimes \llbracket \vec { L } \rangle \rrbracket \left\langle\langle R ^ { \prime } \rrbracket \otimes \llbracket \vec { L } \rangle \rrbracket \left\langle\left\langle L^{\prime}\right\rangle .\right.\right.\right.
$$

This gives a new perspective on the locality arguments for invariance in various forms of Khovanov homology. 
Example II In [9] we computed the type $D$ structure $\llbracket \vec{T}_{L} 》$ for the following tangle $\vec{T}_{L}$, based on the left handed trefoil $T_{L}$ :

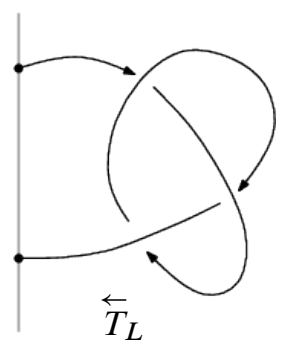

This structure is the map $\vec{\delta}$, where

$$
\begin{aligned}
\vec{\delta}\left(s_{(-3,-15 / 2)}^{+}\right) & =2 \overrightarrow{e_{C}} \otimes s_{(-2,-13 / 2)}^{-}+\overleftarrow{e_{C}} \otimes s_{(-3,-17 / 2)}^{-}, \\
\vec{\delta}\left(s_{(-2,-11 / 2)}^{+}\right) & =-\overleftarrow{e_{C}} \otimes s_{(-2,-13 / 2)}^{-}, \\
\vec{\delta}\left(s_{(0,-3 / 2)}^{+}\right) & =-\overleftarrow{e_{C}} \otimes s_{(0,-5 / 2)}^{-},
\end{aligned}
$$

where the superscript indicates the decoration on the cleaved circle $C$ in the corresponding resolutions, and the subscript is the bigrading. We use the pairing theorem to compute several connected sums.

(i) With the unknot We can think of the unknot $U$ as a cleaved circle on $P_{2}$, where $\overleftarrow{U}=U \cap \overleftarrow{\mathbb{H}}$ and $\vec{U}=U \cap \overrightarrow{\mathbb{H}}$ are the unique planar matchings. Then the left handed trefoil is the connected sum $U \# T_{L}$ which we can think of as gluing $\overleftarrow{U}$ with the tangle above. The type $A$ structure $\left\langle\overleftarrow{U} \rrbracket\right.$ is isomorphic to $\mathbb{Z} f_{(0,1 / 2)} \oplus \mathbb{Z} f_{(0,-1 / 2)}$, which is the idempotent decomposition for $I_{C^{+}}$and $I_{C^{-}}$(see the example in Section 2). The action of $\overleftarrow{e_{C}}$ is trivial since there are no crossings in the standard diagram. On the other hand $\overrightarrow{e_{C}}$ takes $f_{(0,1 / 2)}$ to $f_{(0,-1 / 2)}$. Since $d_{\mathrm{APS}} \equiv 0$ for $\overleftarrow{U}$, we need only compute $\left(m_{2} \otimes \mathbb{I}\right)(\mathbb{I} \otimes \vec{\delta})$. Using the idempotents we see that there are six generators. Furthermore, the only terms in $\left(m_{2} \otimes \mathbb{I}\right)(\mathbb{I} \otimes \vec{\delta})$ come from $\overrightarrow{e_{C}}$. This gives the following chain complex for $\left.\left\langle\overleftarrow{U} \rrbracket \otimes \llbracket \vec{T}_{L}\right\rangle\right\rangle$ :

$$
\begin{aligned}
& f_{(0,1 / 2)} \otimes s_{(-3,-15 / 2)}^{+} \stackrel{\cdot 2}{\rightarrow} f_{(0,-1 / 2)} \otimes s_{(-2,-13 / 2)}^{-}, \\
& f_{(0,1 / 2)} \otimes s_{(-2,-11 / 2)}^{+} \rightarrow 0, \\
& f_{(0,1 / 2)} \otimes s_{(0,-3 / 2)}^{+} \rightarrow 0, \\
& f_{(0,-1 / 2)} \otimes s_{(0,-5 / 2)}^{-} \rightarrow 0, \\
& f_{(0,-1 / 2)} \otimes s_{(-3,-17 / 2)}^{-} \rightarrow 0,
\end{aligned}
$$


whose homology consists of a $\mathbb{Z}$-summand in bigradings $(-2,-5),(0,-1),(0,-3)$, $(-3,-9)$ and a $\mathbb{Z} / 2 \mathbb{Z}$ summand in bigrading $(-2,-7)$. This is the Khovanov homology of the left handed trefoil.

(ii) With the positive Hopf tangle in Section 6 To compute the Khovanov homology of the connected sum of the left handed trefoil with the Hopf link with +1 linking number, recall that the Hopf tangle with positive crossings has $\langle H \rrbracket$ with the following action:

$$
r_{(0,3 / 2)} \stackrel{\overrightarrow{e_{C}}}{\longrightarrow} r_{(0,1 / 2)} \text { and } r_{(2,11 / 2)} \stackrel{\overrightarrow{e_{C}}}{\longrightarrow} r_{(2,9 / 2)},
$$

where the first entry in each corresponds to the + decoration. As a consequence, $\left\langle\left\langle H \rrbracket \otimes_{\mathcal{I}} \llbracket T_{L}\right\rangle\right\rangle$ has the following generators:

$$
\begin{array}{ccc}
r_{(0,3 / 2)} \otimes s_{(-3,-15 / 2)}^{+}, & r_{(2,11 / 2)} \otimes s_{(-3,-15 / 2)}^{+}, & r_{(0,3 / 2)} \otimes s_{(-2,-11 / 2)}^{+}, \\
r_{(2,11 / 2)} \otimes s_{(-2,-11 / 2)}^{+}, & r_{(0,3 / 2)} \otimes s_{(0,-3 / 2)}^{+}, & r_{(2,11 / 2)} \otimes s_{(0,-3 / 2)}^{+}, \\
r_{(0,1 / 2)} \otimes s_{(-2,-13 / 2)}^{-}, & r_{(2,9 / 2)} \otimes s_{(-2,-13 / 2),}^{-}, & r_{(0,1 / 2)} \otimes s_{(-3,-17 / 2)}^{-}, \\
r_{(2,9 / 2)} \otimes s_{(-3,-17 / 2)}^{-}, & r_{(0,1 / 2)} \otimes s_{(0,-5 / 2)}^{-}, & r_{(2,9 / 2)} \otimes s_{(0,-5 / 2)}^{-} .
\end{array}
$$

Since $d_{\mathrm{APS}} \equiv 0$ after the simplification, we need only compute $\left(m_{2} \otimes \mathbb{I}\right)(\mathbb{I} \otimes \vec{\delta})$ on these twelve generators. Since the action $m_{2}$ is trivial except for on $\overrightarrow{e_{C}}$, we can ignore all the terms in $\vec{\delta}$ except for those with $\overrightarrow{e_{C}}$. This leaves the following as the only non-trivial maps in $\partial^{\bigotimes}$ :

$$
\begin{gathered}
r_{(0,3 / 2)} \otimes s_{(-3,-15 / 2)}^{+} \rightarrow 2\left(r_{(0,1 / 2)} \otimes s_{(-2,-13 / 2)}^{-}\right), \\
r_{(2,11 / 2)} \otimes s_{(-3,-15 / 2)}^{+} \rightarrow 2\left(r_{(2,9 / 2)} \otimes s_{(-2,-13 / 2)}^{-}\right) .
\end{gathered}
$$

Taking the homology of this new complex gives two $\mathbb{Z} / 2 \mathbb{Z}$ summands in bigradings $(-2,-6)$ and $(0,-2)$, and eight $\mathbb{Z}$ summands for the remaining generators in the corresponding bigrading:

$$
\begin{array}{ll}
r_{(0,3 / 2)} \otimes s_{(-2,-11 / 2)}^{+} \rightarrow(-2,-4), & r_{(2,11 / 2)} \otimes s_{(-2,-11 / 2)}^{+} \rightarrow(0,0), \\
r_{(0,3 / 2)} \otimes s_{(0,-3 / 2)}^{+} \rightarrow(0,0), & r_{(2,11 / 2)} \otimes s_{(0,-3 / 2)}^{+} \rightarrow(2,4), \\
r_{(0,1 / 2)} \otimes s_{(-3,-17 / 2)}^{-} \rightarrow(-3,-8), & r_{(2,9 / 2)} \otimes s_{(-3,-17 / 2)}^{-} \rightarrow(-1,-4), \\
r_{(0,1 / 2)} \otimes s_{(0,-5 / 2)}^{-} \rightarrow(0,-2), & r_{(2,9 / 2)} \otimes s_{(0,-5 / 2)}^{-} \rightarrow(2,2),
\end{array}
$$

which is isomorphic to the Khovanov homology of the connected sum of the positive Hopf link with the left handed trefoil.

(iii) With a right-handed trefoil We give some details on the computation in the introduction. We consider the tangle $\overleftarrow{T}_{R}$ found by removing an arc from the righthanded trefoil. We can compute $\left\langle\overleftarrow{T}_{R} \rrbracket\right.$ directly. The result is a bigraded module spanned 
by $t_{(0,5 / 2)}^{+}, t_{(2,13 / 2)}^{+}, t_{(3,17 / 2)}^{+}, t_{(0,3 / 2)}^{-}, t_{(2,11 / 2)}, t_{(3,15 / 2)}^{-}$, where the superscript identifies the corresponding idempotent. The action of $\vec{e}_{C}$ is given by $t_{(0,5 / 2)}^{+} \rightarrow t_{(0,3 / 2)}^{-}$, $t_{(2,13 / 2)}^{+} \rightarrow t_{(2,11 / 2)}^{-}, t_{(3,17 / 2)}^{+} \rightarrow t_{(3,15 / 2)}^{-}$. The action of $\overleftarrow{e}_{C}$ is $t_{(2,13 / 2)}^{+} \rightarrow 2 t_{(3,15 / 2)}^{-}$ Consequently, the module $\left\langle\left\langle T_{R} \rrbracket \otimes_{\mathcal{I}} \llbracket \vec{T}_{L}\right\rangle\right.$ has eighteen generators. Those, along with their images under $\partial^{\otimes}$ are shown in the following list:

$$
\begin{aligned}
& t_{(0,5 / 2)}^{+} \otimes s_{(-3,-15 / 2)}^{+} \rightarrow 2 t_{(0,3 / 2)}^{-} \otimes s_{(-2,-13 / 2)}^{-}, \\
& t_{(0,5 / 2)}^{+} \otimes s_{(-2,-11 / 2)}^{+} \rightarrow 0, \\
& t_{(0,5 / 2)}^{+} \otimes s_{(0,-3 / 2)}^{+} \rightarrow 0,
\end{aligned}
$$

$t_{(2,13 / 2)}^{+} \otimes s_{(-3,-15 / 2)}^{+} \rightarrow 2 t_{(2,11 / 2)}^{-} \otimes s_{(-2,-13 / 2)}^{-}+2 t_{(3,15 / 2)}^{-} \otimes s_{(-3,-17 / 2)}^{-}$,

$t_{(2,13 / 2)}^{+} \otimes s_{(-2,-11 / 2)}^{+} \rightarrow-2 t_{(3,15 / 2)}^{-} \otimes s_{(-2,-13 / 2)}^{-}$,

$t_{(2,13 / 2)}^{+} \otimes s_{(0,-3 / 2)}^{+} \rightarrow-2 t_{(3,15 / 2)}^{-} \otimes s_{(0,-5 / 2)}^{-}$,

$t_{(3,17 / 2)}^{+} \otimes s_{(-3,-15 / 2)}^{+} \rightarrow 2 t_{(3,15 / 2)}^{-} \otimes s_{(-2,-13 / 2)}^{-}$,

$t_{(3,17 / 2)}^{+} \otimes s_{(-2,-11 / 2)}^{+} \rightarrow 0, t_{(3,17 / 2)}^{+} \otimes s_{(0,-3 / 2)}^{+} \rightarrow 0$,

$t_{(0,3 / 2)}^{-} \otimes s_{(-3,-17 / 2)}^{-} \rightarrow 0, \quad t_{(0,3 / 2)}^{-} \otimes s_{(-2,-13 / 2)}^{-} \rightarrow 0, \quad t_{(0,3 / 2)}^{-} \otimes s_{(0,-5 / 2)}^{-} \rightarrow 0$,

$t_{(2,11 / 2)}^{\overline{-}} \otimes s_{(-3,-17 / 2)}^{-} \rightarrow 0, t_{(2,11 / 2)}^{-} \otimes s_{(-2,-13 / 2)}^{-} \rightarrow 0, t_{(2,11 / 2)}^{-} \otimes s_{(0,-5 / 2)}^{-} \rightarrow 0$,

$t_{(3,15 / 2)}^{-} \otimes s_{(-3,-17 / 2)}^{-} \rightarrow 0, t_{(3,15 / 2)}^{-} \otimes s_{(-2,-13 / 2)}^{-} \rightarrow 0, t_{(3,15 / 2)}^{-} \otimes s_{(0,-5 / 2)}^{-} \rightarrow 0$.

From this we see immediately that there are $\mathbb{Z}$ summands for each of

- $t_{(0,5 / 2)}^{+} \otimes s_{(-2,-11 / 2)}^{+}$in bigrading $(-2,-3)$,

- $t_{(0,5 / 2)}^{+} \otimes s_{(0,-3 / 2)}^{+}$in $(0,1)$,

- $t_{(3,17 / 2)}^{+} \otimes s_{(-2,-11 / 2)}^{+}$in $(1,3)$,

- $t_{(3,17 / 2)}^{+} \otimes s_{(0,-3 / 2)}^{+}$in $(3,7)$,

- $t_{(0,3 / 2)}^{\overline{2}} \otimes s_{(-3,-17 / 2)}^{-}$in $(-3,-7)$,

- $t_{(0,3 / 2)}^{-} \otimes s_{(0,-5 / 2)}^{-}$in $(0,-1)$,

- $t_{(2,11 / 2)} \otimes s_{(-3,-17 / 2)}^{-}$in $(-1,-3)$, and

- $t_{(2,11 / 2)} \otimes s_{(0,-5 / 2)}^{-}$in $(2,3)$.

The remaining generators occur in the non-zero rows for $\partial^{\bigotimes}$. We will have a $\mathbb{Z} / 2 \mathbb{Z}-$ summand for $t_{(3,15 / 2)}^{\overline{1}} \otimes s_{(0,-5 / 2)}^{\bar{n}}$ in $(3,5)$ and for $t_{(0,3 / 2)} \otimes s_{(-2,-13 / 2)}^{-}$in $(-2,-5)$. 
The equality

$$
\partial^{\bigotimes}\left(t_{(2,13 / 2)}^{+} \otimes s_{(-3,-15 / 2)}^{+}\right)=2 t_{(2,11 / 2)}^{-} \otimes s_{(-2,-13 / 2)}^{-}+2 t_{(3,15 / 2)}^{-} \otimes s_{(-3,-17 / 2)}^{-}
$$

gives a $\mathbb{Z} \oplus \mathbb{Z} / 2 \mathbb{Z}$ in $(0,-1)$. In addition, that

$$
\begin{aligned}
& \partial^{\bigotimes}\left(t_{(2,13 / 2)}^{+} \otimes s_{(-2,-11 / 2)}^{+}\right)=-2 t_{(3,15 / 2)}^{-} \otimes s_{(-2,-13 / 2)}^{-} \quad \text { and } \\
& \partial^{\bigotimes}\left(t_{(3,17 / 2)}^{+} \otimes s_{(-3,-15 / 2)}^{+}\right)=2 t_{(3,15 / 2)}^{-} \otimes s_{(-2,-13 / 2)}^{-}
\end{aligned}
$$

means that $t_{(2,13 / 2)}^{+} \otimes s_{(-2,-11 / 2)}^{+}+t_{(3,17 / 2)}^{+} \otimes s_{(-3,-15 / 2)}^{+}$generates a $\mathbb{Z}$ summand in homology in bigrading $(0,1)$, while $t_{(3,15 / 2)} \otimes s_{(-2,-13 / 2)}^{-}$generates a $\mathbb{Z} / 2 \mathbb{Z}$ summand in $(1,1)$.

Consequently, the Khovanov homology of this connected sum has free part

$$
\mathbb{Z}_{(-3,7)} \oplus \mathbb{Z}_{(-2,-3)} \oplus \mathbb{Z}_{(-1,-3)} \oplus \mathbb{Z}_{(0,-1)}^{2} \oplus \mathbb{Z}_{(0,1)}^{2} \oplus \mathbb{Z}_{(1,3)} \oplus \mathbb{Z}_{(2,3)} \oplus \mathbb{Z}_{(3,7)}
$$

while the torsion part is

$$
(\mathbb{Z} / 2)_{(-2,-5)} \oplus(\mathbb{Z} / 2)_{(0,-1)} \oplus(\mathbb{Z} / 2)_{(1,1)} \oplus(\mathbb{Z} / 2)_{(3,5)} .
$$

This agrees with the Khovanov homology of the knot as computed by Bar-Natan and Green's JavaKh program [4]. Note that we have correctly computed the torsion terms. Furthermore, we provide a modular approach: we can simplify $\llbracket \vec{T} \rrbracket\rangle$ using homotopy equivalences before knowing with which type $D$ structure it will pair.

\section{Appendix: Graded modules, type $A$ structures, conventions}

Summary of the appendix In this appendix, we record the sign conventions used for type $A\left(A_{\infty}\right)$ algebras and modules, review type $D$ structures, and prove the algebraic results about the pairing of these structures. This gives a characteristic-zero account of the algebra in bordered Floer homology [8], where it is stated only in characteristic two. We start by reviewing basics about graded modules and tensor algebras. We then define a category which will allow us to show that the sign conventions we need for this paper will also work with the algebra from [8]. The approach is already present in [8], although not with signs and not as explicitly as we will use it. An object of this category, equipped with a certain differential captures the structural equations for type $A$ structures with the correct sign conventions for this paper. We then describe type $D$ structures in the same language, and show that this description captures the sign conventions use in [9]. We also show that type $D$ structures with these conventions still form an $A_{\infty}$-category. Finally, we define the pairing of our 
type $A$ and type $D$ structures, and prove that the pairing is appropriately functorial for homotopy equivalences in the two factors.

Let $M$ be a $\mathbb{Z}$-graded module over a ring $R$ and let $M_{i}$ be the module of elements in grading $i \in \mathbb{Z}$. For a homogeneous element $m \in M,|m|$ will denote the grading of $m:$ if $m \in M_{i}$ then $|m|=i$.

A module map $f: M \rightarrow M^{\prime}$ has order $r$ if the composition $M_{i} \hookrightarrow M \stackrel{f}{\longrightarrow} M^{\prime}$ has image in $M_{i+r}$ for each $i \in \mathbb{Z}$.

Degree shift convention If $M$ is a $\mathbb{Z}$-graded module, $M[n]$ is the graded module with $(M[n])_{i}=M_{i-n}$, ie the module found by shifting the homogeneous elements of $M$ up $n$ levels. If $m \in M$, the corresponding element in $M[n]$ will be denoted $m[n]$. Thus $|m[n]|=|m|+n$.

An order $r$ map $f: M \rightarrow M^{\prime}$ induces order 0 maps $M \rightarrow M^{\prime}[-r]$ and $M[r] \rightarrow M^{\prime}$, along with maps of different orders $M[n] \rightarrow M[s]$. These will also be denoted by $f$, except where confusion could arise.

The identity on $M$ will be denoted $\mathbb{I}_{M}$. We will also have need of a graded version of the identity.

Definition A.1 $\left|\mathbb{I}_{M}\right|: M \rightarrow M$ is the 0 -order map defined by setting

$$
\left|\mathbb{I}_{M}\right|(m)=(-1)^{|m|} m
$$

for homogeneous $m \in M$ and linearly extending to $M$. $\left|\mathbb{I}_{M}\right|^{j}$ is the $j$-fold composition of $\left|\mathbb{I}_{M}\right|$.

If $m \in M_{i}$ then $\left|\mathbb{I}_{M}\right|^{j}(m)=(-1)^{i j} m$. Consequently, $\left|\mathbb{I}_{M}\right|^{j} \circ\left|\mathbb{I}_{M}\right|^{k}=\left|\mathbb{I}_{M}\right|^{j+k}$ while $\left(\left|\mathbb{I}_{M}\right|^{j}\right)^{k}=\left|\mathbb{I}_{M}\right|^{j k}$.

In addition, shifting changes the sign:

$$
\left|\mathbb{I}_{M[n]}\right|=(-1)^{n}\left|\mathbb{I}_{M}\right| \quad \text { and } \quad\left|\mathbb{I}_{M[n]}\right|^{j}=(-1)^{j n}\left|\mathbb{I}_{M}\right| \text {. }
$$

\section{A.1 Tensor algebras}

We fix a $\mathbb{Z}$-graded $R$-module $A$. As usual,

$$
\mathcal{T}^{*}(A)=\bigoplus_{i=0}^{\infty} A^{\otimes n},
$$

where $A^{\otimes 0}=R$ and for $n>0, A^{\otimes n}=A \otimes_{R} A \otimes_{R} \cdots \otimes_{R} A$ using exactly $n$ factors. $A^{\otimes n}$ is graded using the standard rule $\left|a_{1} \otimes \cdots \otimes a_{n}\right|=\sum\left|a_{i}\right|$. 
Furthermore, $\mathcal{T}^{*}(A)$ has a filtration

$$
R \subset \mathcal{T}^{1}(A) \subset \cdots \subset \mathcal{T}^{k}(A) \subset \cdots,
$$

where $\mathcal{T}^{k}(A)=\bigoplus_{i=0}^{k} A^{\otimes n}$.

By $\mathbb{I}_{A}^{\otimes n}$ we will mean the identity on $A^{\otimes n}$ thought of as the map $\mathbb{I}_{A} \otimes \mathbb{I}_{A} \otimes \cdots \otimes \mathbb{I}_{A}$. In general, we will only use the subscript when we need to distinguish $A$; by default, $\mathbb{I}^{\otimes n}$ will be the identity on $A^{\otimes n}$. Furthermore, by $|\mathbb{I}|^{j \otimes n}$ we will mean the map $|\mathbb{I}|^{j} \otimes \cdots \otimes|\mathbb{I}|^{j}$ on $A^{\otimes n}$.

Definition A.2 For any $\mathbb{Z}$-graded module, $\mathcal{T}_{A}^{*}(M)$ is the $\mathbb{Z}$-graded $R$-module $M \otimes_{R} \mathcal{T}^{*}(A)$ filtered by the submodules $M \otimes \mathcal{T}^{k}(A)$ for $k=0,1,2, \ldots$

Definition A.3 Let $\mathfrak{T}_{A}$ be the category whose objects are the $R$-modules $\mathcal{T}_{A}^{*}(M)$ for each $\mathbb{Z}$-graded module $M$, and whose morphisms, $\mathcal{T}_{A}\left(M, M^{\prime}\right)$, are filtered $R$-module $\operatorname{maps} \Phi: \mathcal{T}_{A}^{*}(M) \rightarrow \mathcal{T}_{A}^{*}\left(M^{\prime}\right)$.

Definition A.4 Let $\Phi \in \mathcal{T}_{A}\left(M, M^{\prime}\right)$. For $i, j \in \mathbb{N}$, the $i j^{\text {th }}$ component of $\Phi$ is the map

$$
\Phi_{i j}: M \otimes A^{\otimes(i-1)} \hookrightarrow \mathcal{T}_{A}^{*}(M) \stackrel{\Phi^{*}}{\longrightarrow} \mathcal{T}^{*}\left(M^{\prime}\right) \rightarrow M^{\prime} \otimes A^{\otimes(j-1)}
$$

Since $\Phi$ is filtered, $\Phi_{i j}=0$ unless $j \leq i$.

\section{A.2 The INF-subcategory of $\mathcal{T}_{A}^{*}$}

Proposition A.5 Let $\mathcal{C}_{A}\left(M, M^{\prime}\right) \subset \mathcal{T}_{A}\left(M, M^{\prime}\right)$ be those module maps $\Phi: \mathcal{T}_{A}^{*}(M) \rightarrow$ $\mathcal{T}_{A}^{*}\left(M^{\prime}\right)$ such that $\Phi$ has order $r$ for some $r \in \mathbb{Z}$, and

$$
\Phi_{n m}=\Phi_{n-m+1,1} \otimes|\mathbb{I}|^{(n+m+r) \otimes(m-1)}
$$

for every $n, m \in \mathbb{N}$ with $1 \leq m \leq n$. Then $\mathcal{C}_{A}\left(M, M^{\prime}\right)$ are the sets of morphisms for a subcategory $\mathcal{C}_{A}$ of $\mathcal{T}_{A}$ whose objects are all the objects of $\mathcal{T}_{A}$.

Proof First, we verify that $\mathbb{I}_{\mathcal{T}_{A}^{*}(M)} \in \mathcal{C}_{A}(M, M) . I_{n m}$ is non-zero only if $n=m$. When $n=m$ the right side of (39) equals $\mathbb{I}_{11} \otimes|\mathbb{I}|^{(n+n+0) \otimes(n-1)}$. However, $I_{11}=\mathbb{I}_{M}$, and $|\mathbb{I}|^{(n+n+0) \otimes(n-1)}=\mathbb{I}^{\otimes(l-1)}$ since an even entry in the first factor in the exponent of $|\mathbb{I}|$ will not change the sign. Thus, $I_{n n}=\mathbb{I}_{M} \otimes \mathbb{I}^{\otimes(n-1)}$, which is the identity on $M \otimes A^{\otimes(n-1)}$. On the other hand, if $n>m$, then $I_{n m} \equiv 0$ and $I_{n-m+1,1} \equiv 0$ as well. Thus, $\mathbb{I}_{\mathcal{T}_{A}^{*}(M)} \in \mathcal{C}_{A}(M, M)$ for every $M$.

We now need to verify that composition of morphisms with the property in (39) will still have this property. Suppose $\Phi \in \mathcal{C}_{A}\left(M, M^{\prime}\right)$ has order $r$ and $\Psi \in \mathcal{C}_{A}\left(M^{\prime}, M^{\prime \prime}\right)$ 
has order $s$, and components of each satisfy (39). Then the order $(r+s)$ morphism $\Psi \circ \Phi$ has components

(40) $(\Psi \circ \Phi)_{n m}$

$$
\begin{aligned}
& =\sum_{m \leq k \leq n} \Psi_{k m} \circ \Phi_{n k} \\
& =\sum_{m \leq k \leq n}\left(\Psi_{k-m+1,1} \otimes|\mathbb{I}|^{(k+m+s) \otimes(m-1)}\right) \circ\left(\Phi_{n-k+1,1} \otimes|\mathbb{I}|^{(n+k+r) \otimes(k-1)}\right) \\
& =\sum_{m \leq k \leq n} \Psi_{k-m+1,1}\left(\Phi_{n-k+1,1} \otimes|\mathbb{I}|^{(n+k+r) \otimes(k-m)}\right) \otimes|\mathbb{I}|^{(n+m+r+s) \otimes(m-1)} .
\end{aligned}
$$

If we let $i=k-m+1$ and $j=n-k+1$, then $n+k \equiv j+1$ modulo 2 , as $k$ changes from $m$ to $n, i$ changes from 1 to $n-m+1$, so we can rewrite the previous result as

$$
\begin{aligned}
& (\Psi \circ \Phi)_{n m} \\
& \quad=\left(\sum_{i+j=n-m+2} \Psi_{i, 1}\left(\Phi_{j, 1} \otimes|\mathbb{I}|^{(j+r+1) \otimes(i-1)}\right)\right) \otimes|\mathbb{I}|^{(n+m+(r+s)) \otimes(m-1)} .
\end{aligned}
$$

On the other hand,

$$
\begin{aligned}
(\Psi \circ \Phi)_{n-m+1,1} & =\sum_{1 \leq i \leq n-m+1} \Psi_{i, 1} \circ \Phi_{n-m+1, i} \\
& =\sum_{1 \leq i \leq n-m+1} \Psi_{i, 1}\left(\Phi_{n-m-i+2,1} \otimes|\mathbb{I}|^{(n+m+1+i+r) \otimes(i-1)}\right) \\
& =\sum_{i+j=n-m+2} \Psi_{i, 1}\left(\Phi_{j, 1} \otimes|\mathbb{I}|^{(j+r+1) \otimes(i-1)}\right)
\end{aligned}
$$

when we let $j=n-m+2-i$. Thus $\Psi \circ \Phi$ satisfies (39).

Definition A.6 For $\Phi \in \mathcal{C}\left(M, M^{\prime}\right)$ of order $r$, the core of $\Phi$ is the set of order $r$ module maps $\Phi^{*}=\left\{\phi_{k} \mid n \in \mathbb{N}\right\}$, where $\phi_{k}=\Phi_{k 1}: M \otimes A^{k-1} \rightarrow M^{\prime}$. Given a set of order $r$ module maps $R=\left\{\rho_{k} \mid n \in \mathbb{N}\right\}$ with $\rho_{k}: M \otimes A^{k-1} \rightarrow M^{\prime}$, the extension of $R$ is the map $\bar{R} \in \mathcal{C}\left(M, M^{\prime}\right)$ with components

$$
\bar{R}_{n m}=\rho_{n-m+1} \otimes|\mathbb{I}|^{(n+m+r) \otimes(m-1)} .
$$

The argument in Proposition A.5 shows that these are inverses: for $\Phi \in \mathcal{C}\left(M, M^{\prime}\right)$, $\bar{\Phi}^{*}=\Phi$, while for $R=\left\{\rho_{k} \mid n \in \mathbb{N}\right\},(\bar{R})^{*}$ equals $R$. Consequently, we can describe $\mathcal{C}_{A}$ completely in terms of a composition on the cores $\Phi^{*}$ which directly reflects the usual module map composition for filtered maps on $\mathcal{T}_{A}^{*}(M)$. This allows us to pull the operations of $\mathcal{C}_{A}$ back to the category of $R$-modules. 
Definition A.7 $\mathcal{C}_{A}^{*}$ is the category whose objects are $\mathbb{Z}$-graded $R$-modules, and whose morphisms $\Phi^{*}: M * M^{\prime}$ are sets $\Phi^{*}=\left\{\phi_{i} \mid i \in \mathbb{N}\right\}$ of $R$-module maps $\phi_{i}: M \otimes A^{\otimes(i-1)} \rightarrow M^{\prime}$ such that every $\phi_{j}$ has order $r$ for some $r \in \mathbb{Z}$. The identity $\mathbb{I}_{M}^{*}: M * M$ is the set of 0 -order module maps with $\left(\mathbb{I}_{M}^{*}\right)_{1}=\mathbb{I}_{M}$ and $\left(\mathbb{I}_{M_{i}}^{*}=0\right.$ for $i>1$. The composition of an order $r$ morphism $\Phi^{*}: M * M^{\prime}$ with an order $s$ morphism $\Psi^{*}: M^{\prime} * M^{\prime \prime}$ is the set of order $r+s$ module maps given by

$$
\left(\Psi^{*} * \Phi^{*}\right)_{k}=\sum_{i+j=k+1} \psi_{i}\left(\phi_{j} \otimes|\mathbb{I}|^{(j+r+1) \otimes(i-1)}\right)
$$

for $k=1,2, \ldots$

Proposition A.8 There is a functor $\mathcal{F}: \mathcal{C}_{A}^{*} \rightarrow \mathcal{C}_{A}$ which takes $M \rightarrow \mathcal{T}_{A}^{*}(M)$ and $\Phi^{*}: M * M^{\prime}$ to its extension $\Phi: \mathcal{T}_{A}^{*}(M) \rightarrow \mathcal{T}_{A}^{*}\left(M^{\prime}\right)$.

Proof This result is implied directly by Proposition A.5.

We will generally work in $\mathcal{C}_{A}$, and then pull back our results to $\mathcal{C}_{A}^{*}$.

\section{A.3 INF-structures}

Since $A$ is a $\mathbb{Z}$-graded $R$-module, we may take $M=A$ above. Then $\mathcal{T}_{A}^{*}(A)=$ $A \otimes \mathcal{T}^{*}(A) \cong \bigoplus_{n=1}^{\infty} A^{\otimes n}$. Let $P: \mathcal{T}_{A}(A) \rightarrow \mathcal{T}_{A}^{*}(A)$ be an order $r$ map in $\mathcal{T}_{A}(A, A)$. Then we can form a new map $P+\left(\mathbb{I}_{A} \otimes P\right)$ in $\mathcal{T}_{A}(A, A)$. This is evidently still filtered.

Definition A.9 An INF-algebra structure on $A$ is an order 1 map $D \in \mathcal{T}(A, A)$ such that

(1) $D \circ D=0$, and

(2) $D+(\mathbb{I} \otimes D)$ is in $\mathcal{C}(A, A)$.

For an INF-algebra structure $D$, we will let $\mu=D+\mathbb{I} \otimes D$. Then the core of $\mu$ is a collection of maps $\mu^{*}=\left\{\mu_{i}: A \otimes \mathcal{A}^{\otimes(i-1)} \rightarrow A\right\}$ in $\mathcal{C}_{A}^{*}(A, A)$. When we have a prescribed $\mu$ in mind, we will write $D_{\mu}$ for the corresponding structure.

Definition A.10 A right INF-module $M$ over $\left(A, D_{\mu}\right)$ is a $\mathbb{Z}$-graded module and an order 1 morphism $D_{M} \in \mathcal{T}(M, M)$ such that

(1) $D_{M} \circ D_{M}=0$, and

(2) $D_{M}+\mathbb{I} \otimes D_{\mu}$ is in $\mathcal{C}(M, M)$. 
Notice that $A$ with the map $D_{\mu}$ is a right module over $\left(A, D_{\mu}\right)$. A right INF-module over $\left(A, D_{\mu}\right)$ is a chain complex with an additional requirement placed on its boundary map. We can similarly adapt the notion of chain map and chain homotopy to this context.

Definition A.11 An INF-module map between right INF-modules $\left(M, D_{M}\right)$ and $\left(M^{\prime}, D_{M^{\prime}}\right)\left(\right.$ over $\left.\left(A, D_{\mu}\right)\right)$ is an order 0 morphism $\Psi \in \mathcal{C}\left(M, M^{\prime}\right)$ such that $\Psi \circ D_{M}=$ $D_{M^{\prime}} \circ \Psi$.

Definition A.12 An INF-homotopy between INF-module maps $\Phi$ and $\Psi$, each mapping $\left(M, D_{M}\right)$ to $\left(M^{\prime}, D_{M^{\prime}}\right)$, is an order -1 map $H \in \mathcal{C}\left(M, M^{\prime}\right)$ such that $\Phi-\Psi=H \circ D_{M}+D_{M^{\prime}} \circ H$.

Since chain complexes form a category, and the maps are drawn from the morphisms of $\mathcal{C}\left(M, M^{\prime}\right)$, we obtain a category of right INF-modules. Furthermore, we can quotient by chain homotopies to obtain a notion of chain homotopy equivalence.

\section{A.4 INF-structures in terms of the core category}

Let $\left(A, D_{\mu}\right)$ be an INF-algebra. The following identity is an immediate consequence of the definition

$$
D_{\mu}=\mu-\left(\mathbb{I} \otimes D_{\mu}\right) .
$$

From this identity we obtain

$$
D_{\mu}=\mu-\mathbb{I} \otimes \mu+\mathbb{I} \otimes \mathbb{I} \otimes \mu+\cdots=\sum_{l=0}^{\infty}(-1)^{l}(\mathbb{I} \otimes l \otimes \mu) .
$$

Note that the sum is actually finite on any summand $A^{\otimes n}$.

If we wish to write out the relations for INF-algebras, modules, morphisms, etc, in terms of their cores, we encounter the difficulty that $D_{\mu}$ is not itself in $\mathcal{C}_{A}(A, A)$. Furthermore, composing with it is not likely to be in $\mathcal{C}_{A}(A, A)$, either. However, a graded commutator with $I \otimes D_{\mu}$ will be an extension. Before we prove this, we must understand commutators with $|\mathbb{I}|^{j}$.

Proposition A.13 If $R \in \mathcal{C}_{A}(A, A)$ has order $r$, then

$$
\left|\mathbb{I}_{A}\right|^{j} \circ R_{k, 1}=(-1)^{r j} R_{k, 1} \circ|\mathbb{I}|^{j \otimes k} .
$$


Proposition A.14 If $\Phi \in \mathcal{C}_{A}\left(M, M^{\prime}\right)$ has order $r$, then $\Phi(\mathbb{I} \otimes D)-(-1)^{r}(\mathbb{I} \otimes D) \Phi$ is in $\mathcal{C}_{A}\left(M, M^{\prime}\right)$ and has core $\left\{(\Phi(\mathbb{I} \otimes D))_{n, 1} \mid n \in \mathbb{N}\right\}$.

Proof We compute the components of $(\mathbb{I} \otimes D) \Phi$ :

$((\mathbb{I} \otimes D) \Phi)_{l m}$

$$
\begin{aligned}
= & \sum_{m \leq k \leq l}(\mathbb{I} \otimes D)_{k m} \circ \Phi_{l k} \\
= & \sum_{m \leq k \leq l}\left(\sum_{s=0}^{\infty}(-1)^{s} \mathbb{I} \otimes \mathbb{I}^{s} \otimes \mu_{k-s-1, m-s-1}\right) \circ\left(\Phi_{l-k+1,1} \otimes|\mathbb{I}|^{(l+k+r) \otimes(k-1)}\right) \\
= & \sum_{m \leq k \leq l}\left[\left(\sum_{s=0}^{\infty}(-1)^{s} \mathbb{I} \otimes \mathbb{I}^{s} \otimes \mu_{k-m+1,1} \otimes|\mathbb{I}|^{(k+m+1) \otimes(m-s-2)}\right)\right. \\
= & \sum_{m \leq k \leq l}\left[\sum_{s=0}^{\infty}(-1)^{s+l+k+r}\left(\Phi_{l-k+1,1} \otimes|\mathbb{I}|^{(l+k+r) \otimes(m-1)}\right)\right. \\
= & \sum_{m \leq k \leq l}\left[\sum_{s^{\prime}=l-k}^{\infty}(-1)^{s^{\prime}+r}\left(\Phi_{l-k+1,1} \otimes|\mathbb{I}|^{(l+k+r) \otimes(m-1)}\right)\right.
\end{aligned}
$$$$
\left.\left(\mathbb{I} \otimes \mathbb{I}^{s^{\prime}} \otimes \mu_{k-m+1,1} \otimes|\mathbb{I}|^{(k+m+1) \otimes\left(m+l-s^{\prime}-k-2\right)}\right)\right]
$$$$
=(-1)^{r} \sum_{m \leq k \leq l}\left(\Phi_{l-k+1,1} \otimes|\mathbb{I}|^{(l+k+r) \otimes(m-1)}\right)
$$$$
\left(\sum_{s^{\prime}=l-k}^{\infty}(-1)^{s^{\prime}} \mathbb{I} \otimes \mathbb{I}^{s^{\prime}} \otimes \mu_{k-m+1,1} \otimes|\mathbb{I}|^{(k+m+1) \otimes\left(m+l-s^{\prime}-k-2\right)}\right)
$$

$$
\begin{aligned}
&=-(-1)^{r} \sum_{m \leq k \leq l}\left(\Phi_{l-k+1,1} \otimes|\mathbb{I}|^{(l+k+r) \otimes(m-1)}\right) \\
&\left(\sum_{s^{\prime}=0}^{l-k-1}(-1)^{s^{\prime}} \mathbb{I} \otimes \mathbb{I}^{s^{\prime}} \otimes \mu_{k-m+1,1} \otimes|\mathbb{I}|^{(k+m+1) \otimes\left(m+l-s^{\prime}-k-2\right)}\right) \\
&+(-1)^{r} \sum_{m \leq k \leq l} \Phi_{l+m-k, m}(\mathbb{I} \otimes D)_{l, l+m-k}
\end{aligned}
$$

$=-(-1)^{r}\left[\sum_{m \leq k \leq l} \Phi_{l-k+1,1}\left(\sum_{s^{\prime}=0}^{l-k-1}(-1)^{s^{\prime}}\left(\mathbb{I} \otimes \mathbb{I}^{s^{\prime}} \otimes \mu_{k-m+1,1} \otimes|\mathbb{I}|^{(k+m+1) \otimes\left(l-s^{\prime}-k-1\right)}\right)\right)\right.$

$$
\left.\otimes|\mathbb{I}|^{(l+m+r+1) \otimes(m-1)}\right]+(-1)^{r}(\Phi(\mathbb{I} \otimes D))_{l, m}
$$

However, $\Phi_{l-k+1,1}$ will consume the $M$ factor as well as the first $l-k$ factors of $A$. Since $s^{\prime}$ only has range up to $l-k-1$, the $\mu$ term must feed into an argument 
of $\Phi_{l-k+1,1}$. By the identity (43), $(-1)^{r}(\mathbb{I} \otimes D) \Phi-\Phi(\mathbb{I} \otimes D)$ is the extension of $\left\{\rho_{n}\right\}$, where

$$
\begin{aligned}
\rho_{n} & =-\sum_{1 \leq k \leq n} \Phi_{n-k+1,1}\left(\sum_{s=0}^{n-k-1}(-1)^{s}\left(\mathbb{I} \otimes \mathbb{I}^{s} \otimes \mu_{k, 1} \otimes|\mathbb{I}|^{k \otimes(n-s-k-1)}\right)\right) \\
& =\sum_{1 \leq k \leq n} \Phi_{n-k+1,1}\left(\sum_{s=0}^{n-k-1}(-1)^{s}\left(\mathbb{I} \otimes \mathbb{I}^{s} \otimes \mu_{n-s-1, n-s-k}\right)\right) .
\end{aligned}
$$

Thus $\rho_{n}=(-\Phi(\mathbb{I} \otimes D))_{n, 1}$.

To write out the requirement that $\left(A, D_{\mu}\right)$ be an INF-algebra in terms of the $\mu_{i}$, we first note that $0=D_{\mu} \circ D_{\mu}=\mu \circ \mu-\left(\mathbb{I} \otimes D_{\mu}\right) \mu-\mu\left(\mathbb{I} \otimes D_{\mu}\right)$. Using the preceding proposition, $0=\mu \circ \mu-\overline{\left\{\left(\mu\left(\mathbb{I} \otimes D_{\mu}\right)\right)_{n 1}\right\}}$. However, $\mu \circ \mu=\overline{\mu^{*} * \mu^{*}}$, so for each $n \in \mathbb{N},(\mu * \mu)_{n}-\left(\mu\left(\mathbb{I} \otimes D_{\mu}\right)\right)_{n, 1}=0$. Unpacking the definitions above produces the following:

$$
\sum_{\substack{i+j=n+1 \\ 1 \leq l \leq i}} \mu_{i}\left(\mu_{j} \otimes|\mathbb{I}|^{j \otimes(i-l)}\right)-\sum_{\substack{i+j=n+1 \\ 0 \leq l \leq i-2}}(-1)^{l} \mu_{i}\left(\mathbb{I} \otimes \mathbb{I}^{\otimes l} \otimes \mu_{j} \otimes|\mathbb{I}|^{j \otimes(i-l-2)}\right)=0,
$$

which is equivalent to

$$
\sum_{\substack{i+j=n+1 \\ 1 \leq l \leq i}}(-1)^{l+1} \mu_{i}\left(\mathbb{I}^{\otimes(l-1)} \otimes \mu_{j} \otimes|\mathbb{I}|^{j \otimes(i-l)}\right)=0 .
$$

Definition A.15 An $A_{\infty}$-algebra structure on a $\mathbb{Z}$-graded $R$-module $A$ is an INFalgebra structure $D_{\mu}$ on $A[-1]$.

If $\mu^{*}=\left\{\mu_{i}\right\}$, then $\mu_{i}:(A[-1])^{\otimes i} \rightarrow A[-1]$ being order 1 means $\mu_{i}:\left((A[-1])^{\otimes i}\right)_{k} \rightarrow$ $(A[-1])_{k+1}$ or $\left(A^{\otimes i}\right)_{k+i} \rightarrow A_{k+2}$. If we let $k^{\prime}=k+i$ then $\mu_{i}: A_{k^{\prime}}^{\otimes i} \rightarrow A_{k^{\prime}-i+2}=$ $A[i-2]_{k^{\prime}}$. Thus, $\mu_{n}$ is a grading preserving map $A^{\otimes n} \rightarrow A[n-2]$. Alternatively, in terms of $A$ with its original grading, each $\mu_{n}$ needs to be an order $2-n$ map $A^{\otimes n} \rightarrow A$.

The preceding relation for an INF-structure on $A[-1]$ is

$$
\sum_{\substack{i+j=n+1 \\ 1 \leq l \leq i}}(-1)^{l+1} \mu_{i}\left(\mathbb{I}^{\otimes(l-1)} \otimes \mu_{j} \otimes|\mathbb{I}|_{A[-1]}^{j \otimes(i-l)}\right)=0 .
$$

Since $|\mathbb{I}|_{A[-1]}=-|\mathbb{I}|_{A}$ in terms of the grading on $A$ we obtain

$$
\sum_{\substack{i+j=n+1 \\ 1 \leq l \leq i}}(-1)^{j(i-l)+l+1} \mu_{i}\left(\mathbb{I}^{\otimes(l-1)} \otimes \mu_{j} \otimes|\mathbb{I}|_{A}^{j \otimes(i-l)}\right)=0 .
$$


However, $j(i-l)+l+1 \equiv j i+l j+l+1 \equiv j(i+1)+(l+1)(j+1)$ so

$$
\sum_{\substack{i+j=n+1 \\ 1 \leq l \leq i}}(-1)^{j(i+1)+(j+1)(l+1)} \mu_{i}\left(\mathbb{I}^{\otimes(l-1)} \otimes \mu_{j} \otimes|\mathbb{I}|_{A}^{j \otimes(i-l)}\right)=0 .
$$

We therefore see that our definition of an $A_{\infty}$-algebra is equivalent to the standard one.

Definition A.16 An $A_{\infty}$-algebra $A$ over a ring $R$ is a $\mathbb{Z}$-graded $R$-module $A$ equipped with maps $\mu_{n}: A^{\otimes n} \rightarrow A[n-2]$ for each $n \in \mathbb{N}$, which satisfy the relation

$$
0=\sum_{\substack{i+j=n+1 \\ l \in\{1, \ldots, i\}}}(-1)^{j(i+1)+(j+1)(l+1)} \mu_{i}\left(\mathbb{I}^{\otimes(l-1)} \otimes \mu_{j} \otimes|\mathbb{I}|^{j \otimes(i-l)}\right) .
$$

Definition A.17 A (right) $A_{\infty}$-module structure on a $\mathbb{Z}$-graded $R$-module $M$, over a $A_{\infty}$-algebra $(A, \mu)$, is a (right) INF-module structure $D_{M[-1]}$ on $M[-1]$ over $\left(A[-1], D_{\mu}\right)$.

Following the argument above, suppose the core of $D+(\mathbb{I} \otimes D)$ is a set of order 1 maps $m_{i}: M[-1] \otimes(A[-1])^{\otimes(i-1)} \rightarrow M[-1]$ with extension $\bar{m}$. Then $D \circ D \equiv 0$ is equivalent to $\bar{m} \circ \bar{m}-\left(\mathbb{I}_{A[-1]} \otimes D_{\mu}\right) \bar{m}-\bar{m}\left(\mathbb{I}_{A[-1]} \otimes D_{\mu}\right) \equiv 0$. Pushing this identity back to one involving the maps $m_{i}: M \otimes A^{\otimes(i-1)} \rightarrow M[i-2]$ yields the following.

Definition A.18 [8] A right $A_{\infty}$-module $M$ over an $A_{\infty}$-algebra $A$ is a set of maps $\left\{m_{i}\right\}_{i \in \mathbb{N}}$ with $m_{i}: M \otimes A^{\otimes(i-1)} \rightarrow M[i-2]$, and satisfying the following relations for each $n \geq 1$ :

$$
\begin{aligned}
& 0=\sum_{i+j=n+1}(-1)^{j(i+1)} m_{i}\left(m_{j} \otimes|\mathbb{I}|^{j \otimes(i-1)}\right) \\
& \quad+\sum_{i+j=n+1, k>0}(-1)^{k(j+1)+j(i+1)} m_{i}\left(\mathbb{I}^{\otimes k} \otimes \mu_{j} \otimes|\mathbb{I}|^{j \otimes(i-k-1)}\right) .
\end{aligned}
$$

$M$ is said to be strictly unital if for any $\xi \in M, m_{2}\left(\xi \otimes \mathbb{I}_{A}\right)=\xi$, but for $n>1$, $m_{n}\left(\xi \otimes a_{1} \otimes a_{2} \otimes \cdots \otimes a_{n-1}\right)=0$ if any $a_{i}=\mathbb{I}_{A}$.

The definition for an INF-morphism unpacks similarly.

Definition A.19 An $A_{\infty}$-morphism $\Psi$ from $M$ to $M^{\prime}$ over $(A, \mu)$ is an INFmorphism from $\left(M[-1], D_{M[-1]}\right)$ to $\left(M^{\prime}[-1], D_{M^{\prime}[-1]}\right)$ over $\left(A[-1], D_{\mu}\right)$.

The same argument as above allows us to write this requirement in terms of the core maps for $\Psi$, conceived of as order 0 module maps $\psi_{i}: M[-1] \otimes(A[-1])^{\otimes(i-1)} \rightarrow$ $M^{\prime}[-1]$. The requirement that $\Psi \circ D_{M[-1]}=D_{M^{\prime}[-1]} \circ \Psi$ becomes $\Psi \circ \bar{m}-\bar{m}^{\prime} \circ \Psi=$ $\Psi \circ\left(\mathbb{I} \otimes D_{\mu}\right)-\left(\mathbb{I} \otimes D_{\mu}\right) \circ \Psi$. By our proposition, $\Psi \circ\left(\mathbb{I} \otimes D_{\mu}\right)-\left(\mathbb{I} \otimes D_{\mu}\right) \circ \Psi$ is the 
extension of $\left\{\left(\Psi\left(\mathbb{I} \otimes D_{\mu}\right)\right)_{n 1}\right\}$, since $\Psi$ is order 0 . Writing out his relation in terms of the cores, and adjusting $|\mathbb{I}|_{A[-1]}=-|\mathbb{I}|_{A}$ as above yields the standard definition.

Definition A.20 [8] An $A_{\infty}$-morphism $\Psi$ of right $A$-modules $M$ and $M^{\prime}$ is a set of maps $\psi_{i}: M \otimes A^{\otimes(i-1)} \rightarrow M^{\prime}[i-1]$ for $i \in \mathbb{N}$, satisfying

$$
\begin{aligned}
& \sum_{i+j=n+1}(-1)^{(i+1)(j+1)} m_{i}^{\prime}\left(\psi_{j} \otimes|\mathbb{I}|^{(j+1) \otimes(i-1)}\right) \\
& =\sum_{i+j=n+1}(-1)^{j(i+1)} \psi_{i}\left(m_{j} \otimes|\mathbb{I}|^{j \otimes(i-1)}\right) \\
& \quad+\sum_{i+j=n+1, k>0}(-1)^{j(i+1)+k(j+1)} \psi_{i}\left(\mathbb{I}^{\otimes k} \otimes \mu_{j} \otimes|\mathbb{I}|^{j \otimes(i-k-1)}\right) .
\end{aligned}
$$

$\Psi$ is strictly unital if $\psi_{i}\left(\xi \otimes a_{1} \otimes \cdots \otimes a_{i-1}\right)=0$ when $a_{j}=\mathbb{I}_{A}$ for some $j$ and $i>1$. The identity morphism $I_{M}$ is the collection of maps $i_{1}(\xi)=\xi, i_{j}=0$ for $j>1$.

Likewise, if we have two morphisms of $A_{\infty}$-modules $\Phi:\left(M^{\prime}[-1], D_{M^{\prime}[-1]}\right) \rightarrow$ $\left(M^{\prime \prime}[-1], D_{M^{\prime \prime}[-1]}\right)$ and $\Psi:\left(M[-1], D_{M[-1]}\right) \rightarrow\left(M^{\prime}[-1], D_{M^{\prime}[-1]}\right)$ over $(A, \mu)$, when we take their composition $\Phi \circ \Psi$, we can write it in terms of the cores of $\Phi$ and $\Psi$, and then adjust the signed identities to be on $A$. This process gives the following.

Definition A.21 [8] Let $\Psi$ be an $A_{\infty}$-morphism from $M$ to $M^{\prime}$, and let $\Phi$ be an $A_{\infty}$-morphism from $M^{\prime}$ to $M^{\prime \prime}$. The composition $\Phi * \Psi$ is the morphism whose component maps for $n \geq 1$ are

$$
(\Phi * \Psi)_{n}^{1}=\sum_{i+j=n+1}(-1)^{(i+1)(j+1)} \phi_{i}\left(\psi_{j} \otimes|\mathbb{I}|^{(j+1) \otimes(i-1)}\right) .
$$

This is almost the same composition defined in $\mathcal{C}_{A[-1]}^{*}$, but in transferring to $A$, we use $|\mathbb{I}|_{A[-1]}^{(j+1) \otimes(i-1)}=(-1)^{(j+1)(i-1)}|\mathbb{I}|_{A}^{(j+1) \otimes(i-1)}$. This accounts for the additional sign.

Definition A.22 Two $A_{\infty}$-morphisms $\Psi, \Phi$ from $M$ to $M^{\prime}$ over $(A, \mu)$ are homotopic if they are homotopic as INF-morphisms from $\left(M[-1], D_{M[-1]}\right)$ to $\left(M^{\prime}[-1]\right.$, $\left.D_{M^{\prime}[-1]}\right)$ over $\left(A[-1], D_{\mu}\right)$.

If $H$ is a homotopy, it is order 1 . Writing out the conditions in terms of its core, using the commutator proposition, and adjusting the signs in using $|\mathbb{I}|_{A}$ instead of $|\mathbb{I}|_{A[-1]}$ produces an equivalent definition. 
Definition A.23 [8] Let $\Psi, \Phi$ be $A_{\infty}$-morphisms from $M$ to $M^{\prime} . \Psi$ and $\Phi$ are homotopic if there is a set of maps $\left\{h_{i}\right\}$ with $h_{i}: M \otimes A^{\otimes(i-1)} \rightarrow M^{\prime}[i]$ such that

$$
\begin{aligned}
\psi_{i}-\phi_{i}=\sum_{i+j=n+1}(-1)^{(i+1) j} m_{i}^{\prime}\left(h_{j} \otimes|\mathbb{I}|^{j \otimes(i-1)}\right) & \\
& +\sum_{i+j=n+1}(-1)^{(i+1) j} h_{i}\left(m_{j} \otimes|\mathbb{I}|^{j \otimes(i-1)}\right) \\
& +\sum_{i+j=n+1, k>0}(-1)^{k(j+1)+j(i+1)} h_{i}\left(\mathbb{I}^{\otimes k} \otimes \mu_{j} \otimes|\mathbb{I}|^{j \otimes(i-k-1)}\right),
\end{aligned}
$$

and for $i>1, h_{i}\left(\xi \otimes a_{1} \otimes \cdots \otimes a_{i-1}\right)=0$ when $a_{j}=\mathbb{I}_{A}$ for some $j$.

In short, all the notions of an $A_{\infty}$-object $O$ come from the same notion for an INFobject applied to $O[-1]$, and then adjusting the signs on $|\mathbb{I}|_{O[-1]}$ to get a formula without grading shifts.

\section{A.5 Incorporating a factor on the right}

Let $A$ and $N$ be $\mathbb{Z}$-graded $R$-modules (as above). We can lift maps $\mathcal{T}^{*}(A) \rightarrow \mathcal{T}^{*}(A)$ to maps which take account of $N$.

Definition A.24 Let $\Psi: \mathcal{T}^{*}(A) \rightarrow \mathcal{T}^{*}(A)$ be an order $r$ module map. $\Psi_{N}$ is the map $\mathcal{T}^{*}(A) \otimes N \rightarrow \mathcal{T}^{*}(A) \otimes N$ with component maps $A^{\otimes n} \otimes N \rightarrow A^{\otimes m} \otimes N$ given by

$$
\left(\Psi_{N}\right)_{n, m}=\Psi_{n, m} \otimes|\mathbb{I}|_{N}^{n-m+r} .
$$

We can also extend maps with domain $N$.

Definition A.25 Let $\phi: N \rightarrow \mathcal{T}^{*}(A) \otimes N^{\prime}$ be a degree $r$ map with projections $\phi_{i}: N \rightarrow A^{\otimes i} \otimes N^{\prime}$. The extension of $\phi$ is the degree $r$ map $\bar{\phi}: \mathcal{T}^{*}(A) \otimes N \rightarrow$ $\mathcal{T}^{*}(A) \otimes N^{\prime}$ with component $\bar{\phi}_{n m}: A^{\otimes n} \otimes N \rightarrow A^{\otimes m} \otimes N^{\prime}$ given by

$$
\bar{\phi}_{n m}=(-1)^{n r}\left(\mathbb{I}_{A}^{n} \otimes \phi_{m-n}\right)
$$

for $n \leq m$ and 0 otherwise.

Proposition A.26 $\bar{\phi}$ is the extension of $\phi$ if and only if $\bar{\phi}=\phi \oplus(-1)^{r}\left(\mathbb{I}_{A} \otimes \bar{\phi}\right)$ under the isomorphism $\mathcal{T}^{*}(A) \otimes N \cong N \oplus A \otimes \mathcal{T}^{*}(A) \otimes N$.

Proof For $m \geq n>0$ we have

$$
\left(\mathbb{I}_{A} \otimes \bar{\phi}\right)_{n m}=\left(\mathbb{I}_{A} \otimes \bar{\phi}_{n-1, m-1}\right)=(-1)^{(n-1) r}\left(\mathbb{I}_{A} \otimes \mathbb{I}_{A}^{n-1} \otimes \phi_{m-n}\right)=(-1)^{r} \bar{\phi}_{n m} .
$$

If $n=0$ then $\left(\mathbb{I}_{A} \otimes \bar{\phi}\right)_{n m}=0$ but $\bar{\phi}_{0 m}=\phi_{m}$. 
Example (1) We think of $\psi: N \rightarrow A \otimes N$ as a map $N \rightarrow \mathcal{T}^{*}(A) \otimes N$ by setting $\psi_{i}=0$ except for $\psi_{1}=\psi$. In this case, $\bar{\psi}$ only has non-zero entries $\bar{\psi}_{n, n+1}=$ $(-1)^{n r}\left(\mathbb{I}_{A}^{\otimes n} \otimes \psi\right)$.

(2) $\mathbb{I}_{N}: N \rightarrow N$ can be considered as a degree 0 map $\iota: N \rightarrow \mathcal{T}^{*}(A) \otimes N$ by setting $\iota_{i}=0$ except for $\iota_{0}=\mathbb{I}_{N}$. In this case, $\bar{\iota}_{n n}=\mathbb{I}_{A}^{n} \otimes \mathbb{I}_{N}$ while $\bar{\iota}_{n m}=0$ for $n \neq m$. Thus $\bar{\iota}=\mathbb{I}_{\mathcal{T}^{*}(A) \otimes N}$.

We now fix an INF-structure $D_{\mu}$ on $A$. Let $\mu$ be the corresponding map on $\mathcal{T}^{*}(A)$ with core maps $\mu^{*}=\left\{\mu_{i}\right\}$ and extension $\mu_{N}: \mathcal{T}^{*}(A) \otimes N \rightarrow \mathcal{T}^{*}(A) \otimes N$. The core of $\mu_{N}$ is the map $\mu_{N}^{*}: \mathcal{T}^{*}(A) \otimes N \rightarrow A \otimes N$ found by extending each $\mu_{i}$ to $A^{\otimes n} \otimes N \rightarrow A \otimes N$ :

$$
\bigoplus_{n=1}^{\infty}\left(\mu_{n} \otimes\left|\mathbb{I}_{N}\right|^{n}\right) \text {. }
$$

A similar set of identities obtain for these maps, and the extension of $D_{\mu}$.

Proposition A.27 Let $D_{\mu, N}: \mathcal{T}^{*}(A) \otimes N \rightarrow \mathcal{T}^{*}(A) \otimes N$ be the extension of $D_{\mu}$. Then $D_{\mu, N}+\mathbb{I} \otimes D_{\mu, N}=\mu_{N}$, and $D_{\mu, N} \circ D_{\mu, N}=0$.

Proof We know that $\left(D_{\mu}\right)_{n, m}=\mu_{n, m}-\left(\mathbb{I} \otimes D_{\mu}\right)_{n, m}$, while on the other hand, $\left(\mathbb{I} \otimes D_{\mu}\right)_{n, m}=\mathbb{I} \otimes\left(D_{\mu}\right)_{n-1, m-1}$. Thus we have the equation $\left(D_{\mu}\right)_{n, m} \otimes|\mathbb{I}|_{N}^{n-m+1}=$ $\mu_{n, m} \otimes|\mathbb{I}|_{N}^{n-m+1}-\mathbb{I} \otimes\left(D_{\mu}\right)_{n-1, m-1} \otimes|\mathbb{I}|_{N}^{n-m+1}$. Consequently, $\left(D_{\mu, N}\right)_{n, m}=$ $\mu_{n, m} \otimes|\mathbb{I}|_{N}^{n-m+1}-\mathbb{I} \otimes\left(D_{\mu, N}\right)_{n-1, m-1}$. Thus $D_{\mu, N}+\mathbb{I} \otimes D_{\mu, N}=\mu_{N}$.

As a consequence of the proposition,

$$
D_{\mu, N}=\sum_{l=0}^{\infty}(-1)^{l}\left(\mathbb{I}^{\otimes l} \otimes \mu_{N}\right) .
$$

\section{A.6 Type $D$ structures}

Definition A.28 A type $D$ structure on $N$ over $\left(A, D_{\mu}\right)$ is an order 0 map $\Delta: N \rightarrow$ $\mathcal{T}^{*}(A) \otimes N$ such that

(1) $\Delta_{0}=\mathbb{I}_{N}$,

(2) $\left(\mathbb{I}_{A}^{\otimes n} \otimes \Delta_{m}\right) \Delta_{n}=\Delta_{m+n}$, and

(3) $D_{\mu, N} \circ \Delta=0$.

Definition A.29 A type $D$ structure $\Delta$ on $N$ (over $\left(A, D_{\mu}\right)$ ) is bounded if there is an $N \in \mathbb{N}$ such that $\Delta_{n} \equiv 0$ whenever $n \geq N$. 
From now on all type $D$ structures in this paper will be bounded, unless otherwise stated.

If we let $\delta=\Delta_{1}$ then $\left(\mathbb{I}_{A}^{\otimes n} \otimes \Delta_{m}\right) \Delta_{n}=\Delta_{m+n}$ implies that

$$
\Delta_{0}=\mathbb{I}_{N}, \quad \Delta_{1}=\delta, \quad \Delta_{n}=\left(\mathbb{I}^{\otimes(n-1)} \otimes \delta\right) \Delta_{n-1} .
$$

We will denote type $D$ structures by this core map: $(N, \delta)$, where $\delta: N \rightarrow A \otimes N$.

Note that we may also extend $\delta: N \rightarrow A \otimes N$ as the map $\bar{\delta}: \mathcal{T}^{*}(A) \otimes N \rightarrow \mathcal{T}^{*}(A) \otimes N$ with $\bar{\delta}_{n, n+1}=\mathbb{I}_{A}^{\otimes n} \otimes \delta$, and that we can similarly extend $\Delta$.

Proposition A.30 Let $\Delta$ be the map for $\delta$, then $\Delta$ satisfies the following identities:

(1) $(\bar{\Delta} \Delta)_{n}=(n+1) \Delta_{n}$,

(2) $\Delta=\mathbb{I}_{N} \oplus\left(\mathbb{I}_{A} \otimes \Delta\right) \delta$, and

(3) $\Delta=\mathbb{I}_{N} \oplus \bar{\delta} \Delta$.

Proof Item (1) follows by noting that $\left(\mathbb{I}^{k} \otimes \Delta_{l}\right)$ is $\bar{\Delta}_{k, k+l}$ and $\left(\mathbb{I}^{k} \otimes \Delta_{l}\right) \Delta_{k}=\Delta_{l+k}$. In the composition, $l$ and $k$ are independent, so we obtain $\Delta_{n}$ in each of the $(n+1)$ ways we can write $n+1=l+k$ with $l, k \geq 0$. For item (2), note that

$$
\left(\mathbb{I}_{A} \otimes \Delta\right)_{n} \delta=\left(\mathbb{I}_{A} \otimes \Delta_{n-1}\right) \delta=\left(\mathbb{I}^{\otimes(n-1)} \otimes \delta\right)\left(\mathbb{I} \otimes \Delta_{n-2}\right) \delta=\left(\mathbb{I}^{\otimes(n-1)} \otimes \delta\right)\left(\mathbb{I}_{A} \otimes \Delta\right)_{n-1} \delta .
$$

Thus the components of $\left(\mathbb{I}_{A} \otimes \Delta\right) \delta$ follow the same definition as $\Delta$. Furthermore, $\left(\mathbb{I}_{A} \otimes \Delta\right)_{1} \delta=\left(\mathbb{I}_{A} \otimes \mathbb{I}_{N}\right) \delta=\delta$, but $\left(\mathbb{I}_{A} \otimes \Delta\right)_{0}=0$ since there must be at least one $A$-factor. Item (2) follows after adjusting the $0^{\text {th }}$ level to compensate. For item (3), we compute $(\bar{\delta} \Delta)_{0 m}=\bar{\delta}_{m-1, m} \circ \Delta_{m-1}$ for $m \geq 1$. Thus, this component equals $\left(\mathbb{I}^{\otimes(m-1)} \otimes \delta\right) \Delta_{m-1}=\Delta_{m}$ for $m \geq 1$. However, $\Delta_{00}=\mathbb{I}_{N}$.

The definition above uses the map $D_{\mu, N}$, but we can use the identities to replace this condition with one depending solely on the core map $\mu_{N}^{*}$.

Proposition A.31 $(N, \delta)$ being a type $D$ structure for $\left(A, D_{\mu}\right)$ is equivalent to either $\mu_{N} \Delta=0$ or

$$
\mu_{N}^{*} \Delta=\sum_{n=1}^{\infty}\left(\mu_{n} \otimes\left|\mathbb{I}_{N}\right|^{n}\right) \Delta_{n} \equiv 0 .
$$

Proof First, note that $D_{\mu, N} \Delta=\mu_{N} \Delta-\left(\mathbb{I}_{A} \otimes D_{\mu, N}\right) \Delta$. If we replace the second $\Delta$ on the right with $\Delta=\mathbb{I}_{N}+\left(\mathbb{I}_{A} \otimes \Delta\right) \delta$ we will get $D_{\mu, N} \Delta=\mu_{N} \Delta-\left(\mathbb{I}_{A} \otimes D_{\mu, N}\right)\left(\mathbb{I}_{A} \otimes \Delta\right) \delta$ 
since $\left(\mathbb{I}_{A} \otimes \Delta\right)$ is zero on $N \subset \mathcal{T}^{*}(A) \otimes N$. However, $\left(\mathbb{I}_{A} \otimes D_{\mu, N}\right)\left(\mathbb{I}_{A} \otimes \Delta\right)=$ $\left.\left(\mathbb{I}_{A} \otimes D_{\mu, N} \Delta\right)\right)$. So

$$
\mu_{N} \Delta=D_{\mu, N} \Delta+\left(\mathbb{I}_{A} \otimes D_{\mu, N} \Delta\right) \delta .
$$

Thus, when $D_{\mu, N} \Delta=0$, then $\mu_{N} \Delta=0$. On the other hand, if $\mu_{N} \Delta=0$ then $D_{\mu, N} \Delta=-\left(\mathbb{I}_{A} \otimes D_{\mu, N} \Delta\right) \delta$. Iterating this yields $D_{\mu, N} \Delta=\left(\mathbb{I}_{A} \otimes\left(\mathbb{I}_{A} \otimes D_{\mu, N} \Delta\right) \delta\right) \delta$ $=\left(\mathbb{I}_{A}^{\otimes 2} \otimes D_{\mu, N} \Delta\right)\left(\mathbb{I}_{A} \otimes \delta\right) \delta=\left(\mathbb{I}_{A}^{\otimes 2} \otimes D_{\mu, N} \Delta\right) \Delta_{2}$. By induction, we can show that $D_{\mu, N} \Delta=(-1)^{n}\left(\mathbb{I}_{A}^{\otimes n} \otimes D_{\mu, N} \Delta\right) \Delta_{n}$. Since $\delta$ is assumed to be bounded, $\Delta_{n} \equiv 0$ for $n$ large enough. Thus $D_{\mu, N} \Delta=0$ when $\mu \Delta=0$.

To complete the argument, we show that the identity in the proposition is equivalent to $\mu_{N} \Delta=0$ or, more simply, $\mu_{N}^{*} \Delta=0$. It follows from the definition of $\mu$ that

$$
(\mu \Delta)_{0 n}=\sum_{j-i=n-1}\left(\mu_{i} \otimes\left|\mathbb{I}_{A}\right|^{i \otimes(j-i)} \otimes\left|\mathbb{I}_{N}\right|^{i}\right) \Delta_{j}
$$

for $n \geq 1$. But $\Delta_{j}=\bar{\delta}^{j-i} \Delta_{i}$ for $j \geq 1$. Since $\delta$ is order 0 , we then have

$$
(\mu \Delta)_{0 n}=\bar{\delta}^{n-1} \sum_{j-i=n-1}\left(\mu_{i} \otimes\left|\mathbb{I}_{N}\right|^{i}\right) \Delta_{i}
$$

since the later application of $\bar{\delta}$ produces factors on the right of the tensor products of $A$. Rewriting the sum to be in terms of $i=j-n+1$, and noting that any $j \geq n$ is possible, we get

$$
(\mu \Delta)_{0 n}=\bar{\delta}^{n-1} \sum_{i=0}^{\infty}\left(\mu_{i} \otimes\left|\mathbb{I}_{N}\right|^{i}\right) \Delta_{i},
$$

from which the statement in the proposition follows directly.

We now consider maps between type $D$ structures and their compositions.

Definition A.32 Let $(N, \delta)$ and $\left(N^{\prime}, \delta^{\prime}\right)$ be bounded type $D$ structures for $\left(A, D_{\mu}\right)$. An order $r$ type $D$ map $\psi:(N, \delta) \circ \rightarrow\left(N^{\prime}, \delta^{\prime}\right)$ is an order $r$ map of graded modules $\psi: N \rightarrow A \otimes N^{\prime}$.

Definition A.33 Let $\psi_{i}:\left(N_{i}, \delta_{i}\right) \circ \rightarrow\left(N_{i+1}, \delta_{i+1}\right), i=1, \ldots, n$ be order $r_{i}$ type $D$ maps. Define $M_{n}\left(\psi_{n}, \ldots, \psi_{1}\right)$ to be the order $1+\sum r_{i}$ map given by

$$
M_{n}\left(\psi_{n}, \ldots, \psi_{1}\right)=\mu_{N_{n+1}}^{*} \bar{\Delta}_{n+1} \bar{\psi}_{n} \bar{\Delta}_{n} \cdots \bar{\Delta}_{2} \bar{\psi}_{1} \Delta_{1} .
$$

Here is the basic proposition relating the $M_{n}$ compositions to the INF-algebra $(A, \mu)$. 
Proposition A.34 Let $\Delta_{i}, i=1, \ldots, n+1$ be type $D$ structures for $\left(A, D_{\mu}\right)$ and let $\psi_{i}$ be a degree $r_{i}$ map from $\left(N_{i}, \Delta_{i}\right)$ to $\left(N_{i+1}, \Delta_{i+1}\right)$. Then

$$
\begin{aligned}
& D_{\mu, N_{n+1}} \bar{\Delta}_{n+1} \bar{\psi}_{n} \bar{\Delta}_{n} \cdots \bar{\Delta}_{2} \bar{\psi}_{1} \Delta_{1} \\
& =\sum_{\substack{1 \leq i \leq n \\
0 \leq l \leq n-i}}(-1)^{\sum_{p=n-L+2}^{n} \operatorname{deg} \psi_{p}} \bar{\Delta}_{n+1} \bar{\psi}_{n} \bar{\Delta}_{n} \cdots \bar{\Delta}_{n-l+1} \bar{M}_{i} \bar{\Delta}_{n-l-i+1} \cdots \bar{\Delta}_{2} \bar{\psi}_{1} \Delta_{1},
\end{aligned}
$$

where $\bar{M}_{i}=\overline{M_{i}\left(\psi_{n-l}, \ldots, \psi_{n-l-i+1}\right)}$.

Proof We consider the image of $\xi \in N_{1}$ under the map found by alternating the $\psi_{i}$ and the $\Delta_{i}$ :

$$
\bar{\Delta}_{n+1} \bar{\psi}_{n} \bar{\Delta}_{n} \cdots \bar{\Delta}_{2} \bar{\psi}_{1} \Delta_{1}
$$

The image of $\xi$ is then a sum of terms of the form

$$
\epsilon_{k_{1}, k_{2}, \ldots, k_{n+1}} a_{1}^{1} \otimes \cdots \otimes a_{k_{1}}^{1} \otimes \gamma_{1} \otimes a_{1}^{2} \otimes \cdots a_{k_{n}}^{n} \otimes \gamma_{n} \otimes a_{1}^{n+1} \otimes \cdots a_{k_{n+1}}^{n+1} \otimes \xi^{\prime}
$$

where $a_{j}^{i} \in A$, each $\gamma_{i} \in A$ marks the factor coming from a $\psi_{i}$, and $\xi^{\prime}$ is some element of $N_{n+1}$. The sign in front equals

$$
\epsilon_{k_{1}, k_{2}, \ldots, k_{n}}=(-1)^{k_{1} r_{1}+\left(k_{1}+1+k_{2}\right) r_{2}+\cdots+\left(k_{1}+1+\cdots+1+k_{n}\right) r_{n}} .
$$

These signs come from the signs in $\bar{\psi}_{i}$ for each of $i=1, \ldots, n:\left(k_{1}+1+\cdots+1+k_{i}\right) r_{i}$, comes from the number of factors preceding $\psi_{i}$, including the $i-1$ factors arising from $\psi_{j}$ with $j<i$, times the degree of $\psi_{i}$.

To this we will apply the map

$$
D_{\mu, N_{n+1}}=\sum_{\substack{1 \leq i \leq M \\ 0 \leq l \leq M-i}}(-1)^{l}\left(\mathbb{I}^{\otimes l} \otimes \mu_{i} \otimes|\mathbb{I}|^{i \otimes(M-l-i)} \otimes\left|\mathbb{I}_{N_{n+1}}\right|^{i}\right)=0
$$

with $M=k_{1}+1+\cdots+1+k_{n}+1+k_{n+1}$. To simplify the computation let $L$ be the number of $\gamma_{k}$-factors which are after the closing parenthesis for $\mu_{i}$ and $I$ be the number of such factors inside $\mu_{i}$. Finally, let $\chi_{s_{1}, \ldots, s_{n}}=s_{1}+1+s_{2}+\cdots+1+s_{n}$. We will fix the value of $I$ for a minute. After applying $D_{\mu, N_{n+1}}$ we obtain terms of the form

$$
\begin{aligned}
& \epsilon_{k_{1}, k_{2}, \ldots, k_{n}} \cdot(-1)^{\chi_{k_{1}, \ldots, k_{n-L-I}, s}} a_{1}^{1} \otimes \cdots \otimes a_{s}^{n-L-I+1} \\
& \quad \otimes \mu_{i}\left(a_{s+1}^{n-L-I+1} \otimes \cdots \otimes \gamma_{n-L-I+1} \otimes \cdots \otimes \gamma_{n-L} \otimes a_{1}^{n-L+1} \otimes \cdots \otimes a_{s^{\prime}}^{n-L+1}\right) \\
& \left.\quad \otimes\left|a_{s^{\prime}}^{n-L+1}\right|^{i} \otimes \cdots \otimes\left|\gamma_{n}\right|^{i} \otimes\left|a_{1}^{n+1}\right|^{i} \otimes \cdots\left|a_{k_{n+1}+1}^{n+1} \otimes\right| \xi^{\prime}\right|^{i},
\end{aligned}
$$

where the additional sign comes from $(-1)^{l}$ in the definition of $D_{\mu, N}$. 
We now do some sign accounting. First, $\epsilon_{k_{1}, k_{2}, \ldots, k_{n}}=\prod_{t=1}^{n+1}(-1)^{\chi_{k_{1}, \ldots, k_{t}} \cdot r_{t}}$. Consequently, we can use this sign to replace each $\gamma_{u}$ with $(-1)^{\chi_{k_{1}, \ldots, k_{u}} r_{u}} \gamma_{u}$. Note that this is the sign which would be used in an application of $\bar{\psi}_{u}$ in the product above. For $\gamma_{n-L}, \ldots, \gamma_{n-L-I+1}$, however, we rewrite $\chi_{k_{1}, \ldots, k_{u}} r_{u}$ as $\chi_{k_{1}, \ldots, k_{n-L-I}, s} r_{u}+p_{u} r_{u}$. Then $p_{u}$ is the number of factors inside $\mu_{i}$ which precede $\gamma_{u}$. We can then bring the $\chi$-sign from the front into the factors, and rewrite the portion which uses $\mu_{i}$ as

$$
\begin{gathered}
(-1)^{\left(1+\sum_{s=1}^{I} r_{n-L-I+s}\right) \chi_{k_{1}, \ldots, k_{n-L-I}, s}} \\
\mu_{i}\left(a_{s+1}^{n-L-I+1} \otimes \cdots \otimes(-1)^{p_{n-L-I+1} r_{n-L-I+1}} \gamma_{n-L-I+1} \otimes \cdots\right. \\
\left.\otimes(-1)^{p_{n-L} r_{n-L}} \gamma_{n-L} \otimes a_{1}^{n-L+1} \otimes \cdots \otimes a_{s^{\prime}}^{n-L+1}\right) .
\end{gathered}
$$

The sign in front is the same as the sign introduced in extending to get $\bar{M}_{I}\left(\psi_{n-L}, \ldots\right.$, $\left.\psi_{n-L-I+1}\right)$, a degree $1+\sum_{s=1}^{I} r_{n-L-I+s}$ map, after skipping $\chi_{k_{1}, \ldots, k_{n-L-I}, s}$ preceding $A$-factors. Each $p_{u}$ is the number of factors preceding the application of $\psi_{u}$ in $M_{I}\left(\psi_{n-L-I+1}, \ldots, \psi_{n-L}\right)$ before extending. There is another sign which is also added when we change to $\bar{M}_{I}$ : in $\bar{M}_{I}$ we use $\mu_{N}^{*}$ not $\mu^{*}$. The action on $N$-factor introduces another sign: that in $\left|\xi^{\prime}\right|^{i}$ versus $\xi^{\prime}$, where $\xi^{\prime}$ is the term in the $N$-factor coming right after the application of $\mu_{i}$. This sign is $(-1)^{i \operatorname{deg}(\tilde{\xi})}$.

Last we consider the terms on the third line. We note that the sign introduced is -1 raised to $i\left(\sum \operatorname{deg} a_{j}^{l}+\sum \operatorname{deg} \gamma_{t}+\operatorname{deg}\left(\xi^{\prime}\right)\right)$ from the signed identity terms, times $(-1)$ raised to the sum of $\chi_{k_{1}, \ldots, k_{p}} r_{p}$ for $n \geq p \geq n-L+1$. In the INFrelation for $\psi_{j}$ we apply $\bar{\psi}_{r}$ after we have used $\mu_{i}$ to contract $i$ factors to 1 factor. Thus the exponent we need differs from $\chi_{k_{1}, \ldots, k_{p}} r_{p}$ by $(i-1) r_{p}$. This occurs for each of the $n-(n-L+1)+1=L$ factors after $\mu_{i}$. Thus, the sign is different by $(-1)^{\sum_{p=n-L+2}^{n}(i-1) r_{p}}$. In addition, $\sum \operatorname{deg} a_{j}^{l}+\sum \operatorname{deg} \gamma_{t}+\operatorname{deg}\left(\xi^{\prime}\right)$ is $\operatorname{deg}(\tilde{\xi})+\sum_{p=n-L+2}^{n} r_{p}$, where $\tilde{\xi}$ is the result in the $N$-factor immediately after applying $\bar{M}_{i}$. This introduces another $i \sum_{p=n-L+2}^{n} r_{p}$ in the exponent. As a consequence, the sign remaining after combining is $(-1)^{i} \operatorname{deg}(\tilde{\xi})+\sum_{p=n-L+2}^{n} r_{p}$. Combining with the sign above, we are left with $(-1)^{\sum_{p=n-L+2}^{n} r_{p}}$.

Thus $(-1)^{l}\left(\mathbb{I}^{\otimes l} \otimes \mu_{j} \otimes|\mathbb{I}|^{j \otimes(i-l)}\right)$ applied to each term in

$$
\left(\bar{\Delta}_{n+1} \bar{\psi}_{n} \bar{\Delta}_{n} \cdots \bar{\Delta}_{2} \bar{\psi}_{1} \Delta_{1}\right)(\xi)
$$

is the same as a term in

$$
(-1)^{\sum_{p=n-L+2}^{n} r_{p}}\left(\bar{\Delta}_{n+1} \bar{\psi}_{n} \bar{\Delta}_{n} \cdots \bar{\Delta}_{n-L+2} \bar{M}_{J} \bar{\Delta}_{n-L-J+2} \cdots \bar{\Delta}_{2} \bar{\psi}_{1} \Delta_{1}\right)(\xi),
$$

where $\bar{M}_{J}=\bar{M}_{J}\left(\psi_{n-L+1}, \ldots, \psi_{n-L-J+2}\right)$. If we add over all the terms we obtain the desired identity. 
Note that we are interpreting $I=0$ as the case where $\mu_{i}$ is applied solely to $A$-factors which come from $\bar{\Delta} \mathrm{s}$. In the final summation these will all cancel since $\Delta_{i}$ is a type $D$ structure.

We note that for type $D$ morphisms, $\sum_{p=n-L+2}^{n} r_{p}=L-1$ and the signs will mimic the INF-relations used above.

Proposition A.35 The compositions $M_{n}, n \in \mathbb{N}$, satisfy the following INF-relations:

$$
\sum_{\substack{i+j=n+1 \\ 1 \leq l \leq i}}(-1)^{\sum_{p=n-L+2}^{n} \operatorname{deg} \psi_{p}} M_{i}\left(\psi_{n}, \ldots, \psi_{n-l+2}, M_{j}, \psi_{n-l-j+1}, \ldots, \psi_{1}\right)=0,
$$

where $M_{j}=M_{j}\left(\psi_{n-l+1}, \ldots, \psi_{n-l-j+2}\right)$.

Proof We compose $\mu_{N_{n+1}}^{*}$ to $D_{\mu, N_{n+1}} \bar{\Delta}_{n+1} \bar{\psi}_{n} \bar{\Delta}_{n} \cdots \bar{\Delta}_{2} \bar{\psi}_{1} \Delta_{1}$. Since $(A, \mu)$ is an INF-algebra, $\mu_{N_{n+1}}^{*} D_{\mu, N_{n+1}}=0$. On the other hand, using Proposition A.34, we see that this implies

$$
\begin{aligned}
& \mu_{N_{n+1}}^{*}\left(\sum_{\substack{1 \leq i \leq n \\
0 \leq l \leq n-i}}(-1)^{\sum_{p=n-L+2}^{n} \operatorname{deg} \psi_{p}} \bar{\Delta}_{n+1} \bar{\psi}_{n} \bar{\Delta}_{n} \cdots \bar{\Delta}_{n-l+1} \bar{M}_{i} \bar{\Delta}_{n-l-i+1} \cdots \bar{\Delta}_{2} \bar{\psi}_{1} \Delta_{1}\right) \\
& \quad=0
\end{aligned}
$$

where $\bar{M}_{i}=\overline{M_{i}\left(\psi_{n-l}, \ldots, \psi_{n-l-i+1}\right)}$.

Moving $\mu_{N_{n+1}}^{*}$ inside the summation, and then using the definition of $M_{n}$, we obtain the INF-relations we desire.

We now concentrate on $M_{1}$ and $M_{2}$. Note that $M_{2}\left(\psi_{2}, \psi_{1}\right)$ has degree $1+r_{1}+r_{2}$. If we limit $\psi_{i}$ to have degree -1 , then $M_{2}\left(\psi_{2}, \psi_{1}\right)$ will also have degree -1 . Thus $M_{2}$ defines a product on the degree -1 maps. Indeed, the INF-relation on -1 maps has the simpler form

$$
\begin{aligned}
& \sum_{\substack{i+j=n+1 \\
1 \leq l \leq i}}(-1)^{l-1} M_{i}\left(\psi_{n}, \ldots, \psi_{n-l+2}, M_{j}\left(\psi_{n-l+1}, \ldots, \psi_{n-l-j+2}\right), \psi_{n-l-j+1}, \ldots, \psi_{1}\right) \\
& \quad=0
\end{aligned}
$$

From the INF-relations we see that $M_{1}$ is a boundary map. We will call a map $\psi$ with $M_{1}(\psi)=0$ a closed map.

Definition A.36 A type $D$ morphism $\psi:(N, \delta) \circ\left(N^{\prime}, \delta^{\prime}\right)$ is a closed order -1 module map $\psi: N \rightarrow A \otimes N^{\prime}$. 
Proposition A.37 A degree -1 map $\psi: N \rightarrow A \otimes N^{\prime}$ is closed if and only if

$$
D_{\mu, N^{\prime}} \circ \bar{\Delta}^{\prime} \circ \bar{\psi} \circ \Delta \equiv 0 \text {. }
$$

Proof By Proposition A.34, we know

$$
D_{\mu, N^{\prime}} \circ \bar{\Delta}^{\prime} \circ \bar{\psi} \circ \Delta=\bar{\Delta}^{\prime} \overline{M_{1}(\psi)} \Delta .
$$

If $M_{1}(\psi)=0$, then $D_{\mu, N^{\prime}} \circ \bar{\Delta}^{\prime} \circ \bar{\psi} \circ \Delta=0$ since $\overline{M_{1}(\psi)}=0$. On the other hand, if the left hand side is 0 , we get that $\bar{\Delta}^{\prime} \overline{M_{1}(\psi)} \Delta=0$. This map has image in $\mathcal{T}^{*}(A) \otimes N^{\prime}$. If we look at the image in $A \otimes N^{\prime}$, we see that it equals $\bar{\Delta}_{11}^{\prime}{\overline{M_{1}(\psi)}}_{01} \Delta_{00}=M_{1}(\psi)$. Thus, when $D_{\mu, N^{\prime}} \circ \bar{\Delta}^{\prime} \circ \bar{\psi} \circ \Delta=0$ we have $M_{1}(\psi)=0$.

We will now restrict ourselves to degree -1 maps of type $D$ structures. The INFidentity for $n=3$ reduces to

$$
M_{1}\left(M_{2}\left(\psi_{2}, \psi_{1}\right)\right)+M_{2}\left(M_{1}\left(\psi_{2}\right), \psi_{1}\right)-M_{2}\left(\psi_{2}, M_{1}\left(\psi_{1}\right)\right)=0 .
$$

We see from this identity that $M_{2}$ will take closed maps to closed maps, thereby defining a product on the kernel of $M_{1}$. Furthermore, $M_{1}$ is a (signed, right) differential for the composition $-M_{2}$. We formalize this as follows.

Proposition A.38 If $\psi:(N, \delta) \circ \rightarrow\left(N^{\prime}, \delta^{\prime}\right)$ and $\phi:\left(N^{\prime}, \delta^{\prime}\right) \circ\left(N^{\prime \prime}, \delta^{\prime \prime}\right)$ are two type $D$ morphisms, then $M_{2}(\phi, \psi):(N, \delta) \circ\left(N^{\prime \prime}, \delta^{\prime \prime}\right)$ is a type $D$ morphism. The composition $\phi * \psi$ is the type $D$ morphism $-M_{2}(\phi, \psi)$.

We require that $A$ be (strictly) unital with identity $1_{A} \in A_{-1}{ }^{1}$. The identity is a two-sided identity for $\mu_{2}$ (which will map $A_{-1} \otimes A_{-1} \rightarrow A_{-2+1}$ ), but its presence as any argument in the application of another core map $\mu_{i}$ will mean the image is 0 .

Proposition A.39 Let $\mathbb{I}_{(N, \delta)}:(N, \delta) \circ \rightarrow(N, \delta)$ be the map $N \rightarrow A \otimes N$ defined by $x \rightarrow 1_{A} \otimes x$. Then $\mathbb{I}_{(N, \delta)}$ is a type $D$ morphism with $M_{2}\left(\mathbb{I}_{\left(N^{\prime}, \delta^{\prime}\right)}, \psi\right)=\psi$ and $M_{2}\left(\phi, \mathbb{I}_{(N, \delta)}\right)=\phi$. Furthermore, the presence of $I_{\left(N_{i}, \delta_{i}\right)}$ as an argument in $M_{n}, n \geq 3$ results in 0 .

Proof First, $I_{(N, \delta)}$ has degree -1 since $1_{A}$ is in $A_{-1}$. Second, we show that $I_{(N, \delta)}$ is a morphism, ie it is closed for $M_{1}$ :

$$
\mu_{N}^{*} \bar{\Delta} \bar{I}_{(N, \delta)} \Delta=0 .
$$

Note that the image in $A^{\otimes n} \otimes N$ will be non-zero only for $n \geq 1$. If it is non-zero, then its image will be linear combinations of terms with a $1_{A}$ in some factor of $A^{\otimes n}$,

\footnotetext{
${ }^{1}$ Recall that we will let $A$ be $A^{\prime}[-1]$ for some $A^{\prime}$, thus $\left(A^{\prime}[-1]\right)_{-1}=A_{-1+1}^{\prime}=A_{0}^{\prime}$.
} 
due to the presence of $I_{(N, \delta)}$. This factor will be fed into a core map $\mu_{i}$ in $\mu_{N}^{*}$. When $i=1$ or $i>2$, the image will then be zero. The only potentially non-zero terms of the composition applied to $\xi$ are those with $\mu_{2}$. If $\delta(\xi)=\sum c_{i} \otimes x_{i}$ we have

$$
\begin{aligned}
\left(\mu_{2}\right. & \left.\otimes I_{N}\right)\left(-\mathbb{I}_{A} \otimes I_{(N, \delta)}\right) \delta(\xi)+\left(\mu_{2} \otimes I_{N}\right)\left(\mathbb{I}_{A} \otimes \delta\right) I_{(N, \delta)}(\xi) \\
& =\left(\mu_{2} \otimes I_{N}\right)\left(-\mathbb{I}_{A} \otimes I_{(N, \delta)}\right)\left(\sum c_{i} \otimes x_{i}\right)+\left(\mu_{2} \otimes I_{N}\right)\left(\mathbb{I}_{A} \otimes \delta\right)\left(1_{A} \otimes \xi\right) \\
& =\left(\mu_{2} \otimes I_{N}\right)\left(-\sum c_{i} \otimes 1_{A} \otimes x_{i}\right)+\left(\mu_{2} \otimes I_{N}\right)\left(1_{A} \otimes\left(\sum c_{i} \otimes x_{i}\right)\right) \\
& =\left(\mu_{2} \otimes I_{N}\right)\left(\sum\left(1_{A} \otimes c_{i}-c_{i} \otimes 1_{A}\right) \otimes x_{i}\right) \\
& =0 .
\end{aligned}
$$

To verify that $I_{(N, \delta)}$ composes as the identity on both sides, we compute

$$
\mu_{N^{\prime}}^{*} \circ \bar{\Delta}^{\prime} \circ \overline{\mathbb{I}_{(N, \delta)}} \circ \bar{\Delta}^{\prime} \circ \bar{\psi} \circ \Delta .
$$

As above, the strict unitality of the maps $\mu_{i}$ mean that the only terms in this composition which are non-zero will be those which feed two factors of $A$ into $\mu_{N^{\prime}}$. These must come from $\overline{\mathbb{I}_{(N, \delta)}}$ and $\bar{\psi}$. Thus the entire composition collapses to a sum of terms $\left(\mu_{2} \otimes \mathbb{I}_{N}\right)\left(-c_{i} \otimes 1_{A} \otimes x_{i}^{\prime}\right)=-\mu_{2}\left(c_{i}, 1_{A}\right) \otimes x_{i}^{\prime}=-c_{i} \otimes x_{i}^{\prime}$, where $\psi(\xi)=\sum c_{i} \otimes x_{i}^{\prime}$. So $\psi * I_{(N, \delta)}=-M_{2}\left(I_{(N, \delta)}, \psi\right)=\psi$. A similar argument shows that $\mathbb{I}_{(N, \delta)}$ acts as an identity on the left (recalling the order reversal in the product).

To see that $\mathbb{I}_{(N, \delta)}$ in an argument of $M_{n}$ for $n>2$ we note that since there are $n$ morphisms the $\mu_{i}$ map applied will have $i \geq n>2$. Furthermore, at least one argument in that $\mu_{i}$ will come from $\mathbb{I}_{(N, \delta)}$ and thus be $1_{A}$. Since the $\mu_{i}$ form a strictly unital INF-algebra, this means that the result must be 0 .

However, the product $-M_{2}$ is not associative. Instead, again from the INF-relations, $M_{2}$ satisfies the generalized associativity relation

$$
\begin{aligned}
& M_{2}\left(M_{2}\left(\psi_{3}, \psi_{2}\right), \psi_{1}\right)-M_{2}\left(\psi_{3}, M_{2}\left(\psi_{2}, \psi_{1}\right)\right) \\
&=-M_{1}\left(M_{3}\left(\psi_{3}, \psi_{2}, \psi_{1}\right)\right)-M_{3}\left(M_{1}\left(\psi_{3}\right), \psi_{2}, \psi_{1}\right) \\
&+M_{3}\left(\psi_{3}, M_{1}\left(\psi_{2}\right), \psi_{1}\right)-M_{3}\left(\psi_{3}, \psi_{2}, M_{1}\left(\psi_{1}\right)\right) .
\end{aligned}
$$

We can simplify this relation by quotienting by the image of $M_{1}$. To this end we declare equivalent any two -1 -morphisms $\psi$ and $\phi$ if there is a degree -2 map $H: N \rightarrow A \otimes N^{\prime}$ with

$$
\psi-\phi=M_{1}(H)=\mu_{N^{\prime}}^{*} \bar{\Delta}^{\prime} \bar{H} \Delta .
$$

We call such morphisms homotopic, following the terminology in [8]. However, equivalent maps represent the same homology class under $M_{1}$. 
That $M_{1}$ is a differential for $M_{2}$ implies that $M_{2}$ defines a composition on the equivalence classes under homotopy. The generalized associativity relation implies that the composition $M_{2}$ is associative once restricted to equivalence classes. As usual, once we have the INF-structure above, we obtain an INF-structure on the homology: the set of closed morphisms after modding out by homotopy. From the arguments above, and the INF-relations we obtain the following.

Proposition A.40 Let $\mathcal{D}$ be the collection of type $D$ structures $(N, \delta)$ over $\left(A, D_{\mu}\right)$. Let $\operatorname{MOR}\left((N, \delta),\left(N^{\prime}, \delta^{\prime}\right)\right)$ be the homotopy equivalence classes of the set of closed degree -1 type $D$ maps. Then $\mathcal{D}$, with these morphism sets, forms a category if we take

(1) the composition

$$
\operatorname{MOR}\left((N, \delta),\left(N^{\prime}, \delta^{\prime}\right)\right) \otimes_{R} \operatorname{MOR}\left(\left(N^{\prime}, \delta^{\prime}\right),\left(N^{\prime \prime}, \delta^{\prime \prime}\right)\right) \rightarrow \operatorname{MOR}\left((N, \delta),\left(N^{\prime \prime}, \delta^{\prime \prime}\right)\right)
$$

to be induced from $(\phi, \psi) \rightarrow-M_{2}(\psi, \phi)$, and

(2) the identity morphism at $(N, \delta)$ to be $\mathbb{I}_{(N, \delta)}$.

\section{A.7 For $A$ a DGA}

We are interested in the following case: $A^{\prime}$ is such that $A=A^{\prime}[-1]$ has an INF-structure with $\mu_{i}=0$ for $i \geq 3$. This makes $A^{\prime}$ into a differential graded algebra. In this case $M_{i} \equiv 0$ for $i \geq 3$ since these require the use of $\mu_{n}$ for $n \geq i$ due to the number of $A^{\prime}[-1]$-factors involved. Examining the INF-relation we see that $M_{2}$ defines an associative composition on type $D$ morphisms, before quotienting by homotopy. Furthermore, $\mathbb{I}_{(N, \delta)}$ is still the identity map. Thus, in this case, type $D$ structures with type $D$ morphisms form a category before quotienting by the homotopy relation. Modding out by homotopy is then quotienting this category by an ideal.

We can write out the conditions for being a type $D$ structure, a type $D$ morphism, and composition of type $D$ structure in this setting. We note that these only require grading shifts and not changes in sign. First, a type $D$ structure is an order 0 map $\delta: N \rightarrow A^{\prime}[-1] \otimes N \cong\left(A^{\prime} \otimes N\right)[-1]$ satisfying

$$
\mu_{N}^{*} \Delta=\sum_{n=1}^{\infty}\left(\mu_{n} \otimes\left|\mathbb{I}_{N}\right|^{n}\right) \Delta_{n} \equiv 0 .
$$

Since $\mu_{n}=0$ for $n>2$ we can simplify this to

$$
\left(\mu_{2} \otimes \mathbb{I}_{N}\right) \Delta_{2}+\left(\mu_{1} \otimes\left|\mathbb{I}_{N}\right|\right) \Delta_{1}=0 .
$$


Since $\Delta_{2}=\left(\mathbb{I}_{A^{\prime}[-1]} \otimes \delta\right) \delta$ and $\Delta_{1}=\delta$ we obtain the relation

$$
\left(\mu_{2} \otimes \mathbb{I}_{N}\right)\left(\mathbb{I}_{A^{\prime}} \otimes \delta\right) \delta+\left(\mu_{1} \otimes\left|\mathbb{I}_{N}\right|\right) \delta=0 .
$$

By a similar argument, we see that a morphism of type $D$ structure will be an order -1 map $\psi: N \rightarrow\left(A^{\prime}[-1] \otimes N^{\prime}\right)$. Thus, $\psi$ maps $N_{k}$ to $\left(A^{\prime} \otimes N\right)[-1]_{k-1} \cong\left(A^{\prime} \otimes N\right)_{k}$. Thus we can take a type $D$ morphism to be an order 0 map $N \rightarrow A^{\prime} \otimes N^{\prime}$ which satisfies $\mu_{N^{\prime}}^{*} \bar{\Delta}^{\prime} \bar{\psi} \Delta=0$. This simplifies to

$$
\left(\mu_{2} \otimes \mathbb{I}_{N}\right)\left(\bar{\Delta}^{\prime} \bar{\psi} \Delta\right)_{02}+\left(\mu_{1} \otimes\left|\mathbb{I}_{N}\right|\right)\left(\bar{\Delta}^{\prime} \bar{\psi} \Delta\right)_{01}=0 .
$$

$\bar{\psi}$ will increase the number of $A^{\prime}$-factors by one, so $\left(\bar{\Delta}^{\prime} \bar{\psi} \Delta\right)_{01}=\left(\mathbb{I}_{A^{\prime}} \otimes \mathbb{I}_{N^{\prime}}\right) \psi \mathbb{I}_{N}$ since we must use $\Delta_{0}^{\prime}$ and $\Delta_{0}$ or else have too many factors. On the other hand, in the first term we may use either $\Delta_{1}$ or $\Delta_{1}^{\prime}$, but not both. Then

$$
\left(\bar{\Delta}^{\prime} \bar{\psi} \Delta\right)_{02}=\left(\mathbb{I}_{A^{\prime}[-1]} \otimes \delta^{\prime}\right) \psi-\left(\mathbb{I}_{A^{\prime}[-1]} \otimes \psi\right) \delta .
$$

Under our isomorphisms, this becomes

$$
\left(\mu_{2} \otimes \mathbb{I}_{N}\right)\left(\mathbb{I}_{A^{\prime}[-1]} \otimes \delta^{\prime}\right) \psi-\left(\mu_{2} \otimes \mathbb{I}_{N}\right)\left(\mathbb{I}_{A^{\prime}[-1]} \otimes \psi\right) \delta+\left(\mu_{1} \otimes\left|\mathbb{I}_{N}\right|\right) \psi=0 .
$$

The composition of two morphisms $\psi:(N, \delta) \circ\left(N^{\prime}, \delta^{\prime}\right)$ and $\phi:\left(N^{\prime}, \delta^{\prime}\right) \circ\left(N^{\prime \prime}, \delta^{\prime \prime}\right)$ can be computed from $-M_{2}(\phi, \psi)=-\mu_{N^{\prime \prime}}^{*} \bar{\Delta}^{\prime \prime} \bar{\phi} \bar{\Delta}^{\prime} \bar{\psi} \Delta$. Both $\bar{\psi}$ and $\bar{\phi}$ introduce $A^{\prime}[-1]$-factors. Hence, the contributions of $\bar{\Delta}^{\prime \prime}, \bar{\Delta}^{\prime}$ and $\Delta$ must either be the identity on the respective modules, or introduce $A^{\prime}[-1]$-factors which force $\mu_{N^{\prime \prime}}^{*}$ to evaluate to 0 , as $\mu_{i}=0$ for $i>2$. Thus,

$$
-M_{2}(\phi, \psi)=-\left(\mu_{2} \otimes \mathbb{I}_{N^{\prime \prime}}\right)\left(-\mathbb{I}_{A^{\prime}} \otimes \phi\right) \psi=\left(\mu_{2} \otimes \mathbb{I}_{N^{\prime \prime}}\right)\left(\mathbb{I}_{A^{\prime}} \otimes \phi\right) \psi .
$$

Furthermore, a homotopy $H: N \rightarrow\left(A^{\prime}[-1] \otimes N^{\prime}\right)$ is a degree -2 map, and thus can be thought of as a map $N_{k} \rightarrow\left(A^{\prime} \otimes N^{\prime}[-1]\right)_{k-2} \cong\left(A^{\prime} \otimes N^{\prime}[+1]\right)_{k}$. It is thus an order 0 map $N \rightarrow\left(A^{\prime} \otimes N^{\prime}\right)[+1]$. Furthermore, as above, we can compute $M_{1}(H)=\left(\mu_{2} \otimes \mathbb{I}_{N}\right)\left(\bar{\Delta}^{\prime} \bar{H} \Delta\right)_{02}+\left(\mu_{1} \otimes\left|\mathbb{I}_{N}\right|\right)\left(\bar{\Delta}^{\prime} \bar{H} \Delta\right)_{01}$ which simplifies using

$$
\left(\bar{\Delta}^{\prime} \bar{H} \Delta\right)_{02}=\left(\mathbb{I}_{A^{\prime}[-1]} \otimes \delta^{\prime}\right) H+\left(\mathbb{I}_{A^{\prime}[-1]} \otimes H\right) \delta,
$$

since $H$ has even order. Thus if $\psi$ and $\phi$ are homotopic type $D$ morphisms, with homotopy $H$, then

$$
\psi-\phi=\left(\mu_{2} \otimes \mathbb{I}_{N}\right)\left(\mathbb{I}_{A^{\prime}[-1]} \otimes \delta^{\prime}\right) H+\left(\mu_{2} \otimes \mathbb{I}_{N}\right)\left(\mathbb{I}_{A^{\prime}[-1]} \otimes H\right) \delta+\left(\mu_{1} \otimes\left|\mathbb{I}_{N}\right|\right) H,
$$

or, after applying the shift isomorphisms

$$
\psi-\phi=\left(\mu_{2} \otimes \mathbb{I}_{N}\right)\left(\mathbb{I}_{A^{\prime}} \otimes \delta^{\prime}\right) H+\left(\mu_{2} \otimes \mathbb{I}_{N}\right)\left(\mathbb{I}_{A^{\prime}} \otimes H\right) \delta+\left(\mu_{1} \otimes\left|\mathbb{I}_{N}\right|\right) H .
$$




\section{A.8 Pairing}

Since $D_{M, N} \circ D_{M, N}=0$ and $D_{M, N}=m_{N}-\mathbb{I}_{M} \otimes D_{\mu, N}$ we see that $m_{N} m_{N}=$ $m_{N}\left(\mathbb{I}_{M} \otimes D_{\mu, N}\right)+\left(\mathbb{I}_{M} \otimes D_{\mu, N}\right) m_{N} .\left(\mathbb{I}_{M} \otimes D_{\mu, N}\right) m_{N}$ has image in $\bigoplus_{n>0} M \otimes$ $A^{n} \otimes N$ since there is always an $A$-factor remaining in the codomain of $D_{\mu, N}$. Thus, after restricting to have domain and codomain $M \otimes_{R} N$,

$$
m_{N}^{*} \circ m_{N}=m_{N}^{*}\left(\mathbb{I}_{M} \otimes D_{\mu, N}\right) .
$$

This works for any right INF-module $M$.

Now suppose that we have type $D$ structures $\left(N_{i}, \Delta_{i}\right)$ for $i=1, \ldots, n+1$. Let $\psi_{i}:\left(N_{i}, \delta_{i}\right) \circ\left(N_{i+1}, \delta_{i+1}\right), i=1, \ldots, n$ be order $r_{i}$ type $D$ maps. We will now apply both sides of $m_{N_{n+1}}^{*} \circ m_{N_{n+1}}=m_{N_{n+1}}^{*}\left(\mathbb{I}_{M} \otimes D_{\mu, N_{n+1}}\right)$ to

$$
\xi=\mathbb{I}_{M} \otimes\left(\bar{\Delta}_{n+1} \bar{\psi}_{n} \bar{\Delta}_{n} \cdots \bar{\Delta}_{2} \bar{\psi}_{1} \Delta_{1}\right) .
$$

We let

$$
\begin{aligned}
& \Omega_{n}\left(\psi_{n}, \ldots, \psi_{1}\right) \\
& \quad=m_{N_{n+1}}^{*}\left(\mathbb{I}_{M} \otimes \bar{\Delta}_{n+1}\right)\left(\mathbb{I}_{M} \otimes \bar{\psi}_{n}\right)\left(\mathbb{I}_{M} \otimes \bar{\Delta}_{n}\right) \cdots\left(\mathbb{I}_{M} \otimes \bar{\Delta}_{2}\right)\left(\mathbb{I}_{M} \otimes \bar{\psi}_{1}\right)\left(\mathbb{I}_{M} \otimes \Delta_{1}\right)
\end{aligned}
$$

for $n \geq 1$, and $\Omega_{0}=m_{N_{1}}^{*} \Delta_{1}$.

By Proposition A.34,

$$
\begin{aligned}
& m_{N}^{*}\left(\mathbb{I}_{M} \otimes D_{\mu, N}\right)(\xi)= \\
& \quad \sum_{\substack{i=n+1 \\
1 \leq l \leq i}}(-1)^{\sum_{p=n-l+2}^{n} \operatorname{deg} \psi_{p}} \Omega_{i}\left(\psi_{n}, \ldots, \psi_{n-l+2}, M_{j}, \psi_{n-l-j+1}, \ldots, \psi_{1}\right),
\end{aligned}
$$

where $M_{j}=M_{j}\left(\psi_{n-l+1}, \ldots, \psi_{n-l-j+2}\right)$.

Similar to the proof of Proposition A.34 we can analyze $\left(m_{N}^{*} \circ m_{N}\right)(\xi)$. There are two differences between this argument and that in the proof of Proposition A.34. The first occurs in the signs: there we removed $j$ factors of $A$ by applying $\mu_{j}$ and replaced it with a new factor (the image) which resulted in a difference of sign of $(j-1) r_{k}$ for each $\psi_{k}$ occurring after the application of $\mu_{j}$. Here, however, applying $m_{j}$ removes $j-1$ factors and merges them into the $M$ factor out front. This also results in a change of $(j-1) r_{k}$. The second difference is that $m_{N}^{*} \Delta=\Omega_{0}=\partial^{\bigotimes}$ and not zero as before. Furthermore, when we apply $m_{j}$ followed by $m_{i}$ we obtain a composition of $\Omega_{i}$ and $\Omega_{j}$ since each has image in $M \otimes N$. Putting these observations together with the proof of Proposition A.34 we get

$$
\left(m_{N}^{*} \circ m_{N}\right)(\xi)=\sum_{i+j=n}(-1)^{\sum_{p=j+1}^{n} \operatorname{deg} \psi_{p}} \Omega_{i}\left(\psi_{n}, \ldots, \psi_{j+1}\right) \Omega_{j}\left(\psi_{j}, \ldots, \psi_{1}\right),
$$


where both $i$ and $j$ on the left side can equal 0 . Consequently,

$$
\begin{aligned}
& \sum_{i+j=n}(-1)^{\sum_{p=j+1}^{n} \operatorname{deg} \psi_{p}} \Omega_{i}\left(\psi_{n}, \ldots, \psi_{j+1}\right) \Omega_{j}\left(\psi_{j}, \ldots, \psi_{1}\right) \\
& \quad=\sum_{\substack{i+j=n+1 \\
1 \leq l \leq i}}(-1)^{\sum_{p=n-l+2}^{n} \operatorname{deg} \psi_{p}} \Omega_{i}\left(\psi_{n}, \ldots, \psi_{n-l+2}, M_{j}, \psi_{n-l-j+1}, \ldots, \psi_{1}\right),
\end{aligned}
$$

where $M_{j}=M_{j}\left(\psi_{n-l+1}, \ldots, \psi_{n-l-j+2}\right)$.

\section{A.9 Pairing a left INF-module and a type $D$ structure}

Let $\left(A, D_{\mu}\right)$ be an INF-algebra with $\mu^{*}=\left\{\mu_{i}\right\}$, and let $M$ be a right INF-module over $\left(A, D_{\mu}\right)$ with differential $D_{M}$. We let $m=D_{M}+\mathbb{I}_{M} \otimes D_{\mu}$, and $m^{*}=\left\{m_{i}\right\}$ be the corresponding core maps $m_{n}: M \otimes A^{\otimes(n-1)} \rightarrow M$. In addition, we let $(N, \delta)$ be a type $D$ structure over $\left(A, D_{\mu}\right)$.

Definition A.41 Define $M \otimes N$ to be the graded module $M \otimes_{R} N$, and $\partial^{\otimes}: M \otimes N \rightarrow$ $(M \otimes N)[-1]$ to be the map

$$
\partial^{\bigotimes}=m_{N}^{*}\left(\mathbb{I}_{M} \otimes \Delta\right)=\sum_{k=0}^{\infty}\left(m_{k+1} \otimes\left|\mathbb{I}_{N}\right|^{k+1}\right) \circ\left(\mathbb{I}_{M} \otimes \Delta_{k}\right) .
$$

Theorem A.42 [8] $\left(M \otimes N, \partial^{\bigotimes}\right)$ is a chain complex.

Proof We note that $\partial^{\bigotimes}=\Omega_{0}$ for $N_{1}=N$. Taking the relation for $i=j=0$ we obtain $\Omega_{0} \Omega_{0}=0$, since the right hand side contains no terms. This shows that $\partial^{\bigotimes}$ is a boundary map.

Proposition A.43 For each type $D$ structure $(N, \delta)$ over $\left(A, D_{\mu}\right)$ there is a functor $\mathcal{F}_{(N, \delta)}$ from the category of right INF-modules over $\left(A, D_{\mu}\right)$ to the category of chain complexes. $\mathcal{F}_{(N, \delta)}$ is defined by

$$
\mathcal{F}_{(N, \delta)}\left(M, D_{M}\right)=\left(M \otimes N, \partial^{\bigotimes}\right), \quad \mathcal{F}_{(N, \delta)}(\Phi)=\Phi_{N}^{*}\left(\mathbb{I}_{M} \otimes \Delta\right),
$$

where $\Phi \in \mathcal{C}\left(M, M^{\prime}\right)$ is a morphism of right INF-modules over $\left(A, D_{\mu}\right)$. We will denote $\mathcal{F}_{(N, \delta)} \Phi$ by $\Phi \otimes \mathbb{I}_{N}$. Furthermore, if $\Phi$ and $\Psi$ are homotopic then $\Phi \otimes \mathbb{I}_{N}$ is chain homotopic to $\Psi \otimes \mathbb{I}_{N}$. Thus, $\mathcal{F}_{(N, \delta)}$ induces a functor from the homotopy category of right INF-modules to the homotopy category of chain complexes.

Before proving this proposition, we introduce a useful lemma.

Lemma A.44 Let $\Phi \in \mathcal{C}\left(M, M^{\prime}\right)$ have order $r$. Then

$$
\left(\mathbb{I}_{M^{\prime}} \otimes \Delta\right) \circ\left(\Phi_{N}^{*}\right) \circ\left(\mathbb{I}_{M} \otimes \Delta\right)=\Phi_{N} \circ\left(\mathbb{I}_{M} \otimes \Delta\right) .
$$


Proof The image of $\left(\mathbb{I}_{M^{\prime}} \otimes \Delta\right) \circ\left(\Phi_{N}^{*}\right) \circ\left(\mathbb{I}_{M} \otimes \Delta\right)$ in $M \otimes A^{\otimes n} \otimes N$ has the form

$$
\sum_{l}\left(\mathbb{I}_{M^{\prime}} \otimes \Delta_{n}\right)\left(\Phi_{l+1}^{*} \otimes\left|\mathbb{I}_{N}\right|^{l+r}\right)\left(\mathbb{I}_{M} \otimes \Delta_{l}\right)
$$

Since $\left(\mathbb{I}_{M} \otimes \Delta_{n}\right)$ does not change the $M$ factor, and $\left(\Phi_{l+1}^{*} \otimes\left|\mathbb{I}_{N}\right|^{l+r}\right)$ only affects the $M$ factor and the $l$ available $A$-factors, we can rewrite

$\left(\mathbb{I}_{M^{\prime}} \otimes \Delta_{n}\right)\left(\Phi_{l+1}^{*} \otimes\left|\mathbb{I}_{N}\right|^{l+r}\right)$

$$
=\left(\Phi_{l+1}^{*} \otimes \mathbb{I}_{A}^{\otimes n} \otimes \mathbb{I}_{N}\right)\left(\mathbb{I}_{M} \otimes \mathbb{I}_{A}^{\otimes l} \otimes \Delta_{n}\right)\left(\mathbb{I}_{M} \otimes \mathbb{I}_{A}^{\otimes l} \otimes\left|\mathbb{I}_{N}\right|^{l+r}\right) .
$$

Furthermore, as $\Delta_{n}$ preserves grading, so

Therefore,

$$
\Delta_{n}\left(\left|\mathbb{I}_{N}\right|^{l+r}\right)=\left(\left|\mathbb{I}_{A}\right|^{(l+r) \otimes n} \otimes\left|\mathbb{I}_{N}\right|^{l+r}\right) \Delta_{n} .
$$

$\left(\mathbb{I}_{M^{\prime}} \otimes \Delta_{n}\right)\left(\Phi_{l+1}^{*} \otimes\left|\mathbb{I}_{N}\right|^{l+r}\right)$

$$
=\left(\Phi_{l+1}^{*} \otimes \mathbb{I}_{A}^{\otimes n} \otimes \mathbb{I}_{N}\right)\left(\mathbb{I}_{M} \otimes \mathbb{I}_{A}^{\otimes l} \otimes\left|\mathbb{I}_{A}\right|^{(l+r) \otimes n} \otimes\left|\mathbb{I}_{N}\right|^{l+1}\right)\left(\mathbb{I}_{M} \otimes \mathbb{I}_{A}^{\otimes l} \otimes \Delta_{n}\right) .
$$

However,

$\left(\Phi_{l+1}^{*} \otimes \mathbb{I}_{A}^{\otimes n} \otimes \mathbb{I}_{N}\right)\left(\mathbb{I}_{M} \otimes \mathbb{I}_{A}^{\otimes l} \otimes\left|\mathbb{I}_{A}\right|^{(l+r) \otimes n} \otimes\left|\mathbb{I}_{N}\right|^{l+r}\right)=\Phi_{n+l+1, n+1} \otimes\left|\mathbb{I}_{N}\right|^{l+1}$,

which is an entry in $\Phi_{N}$. If we precompose with $\mathbb{I}_{M} \otimes \Delta_{l}$ we can then replace $\left(\mathbb{I}_{M} \otimes \mathbb{I}_{A}^{\otimes l} \otimes \Delta_{n}\right)\left(\mathbb{I}_{M} \otimes \Delta_{l}\right)$ with $\mathbb{I}_{M} \otimes \Delta_{l+n}$. As a consequence, the image in $M \otimes A^{\otimes n} \otimes N$ is the sum $\sum_{l}\left(\Phi_{n+l+1, n+1} \otimes\left|\mathbb{I}_{N}\right|^{l+r}\right)\left(\mathbb{I}_{M} \otimes \Delta_{l+n}\right)$, which is also the entry in $\Phi_{N}\left(\mathbb{I}_{M} \times \Delta\right)$ which maps $M \otimes N$ to $M \otimes A^{\otimes n} \otimes N$.

Proof of Proposition A.43 Let $\Phi \in \mathcal{C}\left(M, M^{\prime}\right)$ be an order 0 morphism from $M$ to $M^{\prime}$ with core $\Phi^{*}=\left\{\phi_{k}\right\}$. We define

$$
\phi \otimes \mathbb{I}_{N}=\Phi_{N}^{*}\left(\mathbb{I}_{M} \otimes \Delta\right) .
$$

To see that this is a chain map, we compute $\left(\Phi \otimes \mathbb{I}_{N}\right) \partial^{\bigotimes}=\Phi_{N}^{*}\left(\mathbb{I}_{M} \otimes \Delta\right)\left(m_{N}^{*}\right)\left(\mathbb{I}_{M} \otimes \Delta\right)$. Using the previous lemma we can simplify this to $\Phi_{N}^{*}\left(m_{N}\right)\left(\mathbb{I}_{M} \otimes \Delta\right)$. Since $\Phi$ is a morphism of right INF-modules, we have $\Phi_{N} D_{M, N}=D_{M^{\prime}, N} \Phi_{N}$, or

$$
\Phi_{N}\left(m_{N}-\left(\mathbb{I}_{M} \otimes D_{\mu, N}\right)\right)=\left(m_{N}^{\prime}-\left(\mathbb{I}_{M} \otimes D_{\mu, N}\right)\right) \Phi_{N} .
$$

If we look at those terms with image in $M \otimes N$ we obtain $\Phi_{N}^{*} m_{N}-\Phi_{N}^{*}\left(\mathbb{I}_{M} \otimes D_{\mu, N}\right)=$ $\left(m_{N}^{\prime}\right)^{*} \Phi_{N}$. Thus

$\left(\Phi \otimes \mathbb{I}_{N}\right) \partial^{\bigotimes}=\left(\Phi_{N}^{*}\left(\mathbb{I}_{M} \otimes D_{\mu, N}\right)+\left(m_{N}^{\prime}\right)^{*} \Phi_{N}\right)\left(\mathbb{I}_{M} \otimes \Delta\right)=\left(m_{N}^{\prime}\right)^{*} \Phi_{N}\left(\mathbb{I}_{M} \otimes \Delta\right)$.

On the other hand, $\partial^{\otimes}\left(\Phi \otimes \mathbb{I}_{N}\right)=\left(m_{N}^{*}\right)\left(\mathbb{I}_{M} \otimes \Delta\right)\left(\Phi_{N}^{*}\right)\left(\mathbb{I}_{M} \otimes \Delta\right)$, which reduces to $\left(m_{N}^{*}\right) \Phi_{N}\left(\mathbb{I}_{M} \otimes \Delta\right)$, using the lemma above. Thus, $\left(\Phi \otimes \mathbb{I}_{N}\right)$ is a chain map. 
Let $\Psi: M \rightarrow M^{\prime}$ and $\Phi: M^{\prime} \rightarrow M^{\prime \prime}$ be morphisms of right INF-modules. Then $(\Phi * \Psi) \otimes \mathbb{I}_{N}$ is the map $(\Phi * \Psi)_{N}^{*}\left(\mathbb{I}_{M} \otimes \Delta\right)=\Phi_{N}^{*} \Psi_{N}\left(\mathbb{I}_{M} \otimes \Delta\right)$. Since $\Psi_{N}\left(\mathbb{I}_{M} \otimes \Delta\right)=$ $\left(\mathbb{I}_{M} \otimes \Delta\right) \Psi_{N}^{*}\left(\mathbb{I}_{M} \otimes \Delta\right)$, we see that $(\Phi * \Psi) \otimes \mathbb{I}_{N}=\Phi_{N}^{*}\left(\mathbb{I}_{M} \otimes \Delta\right) \Psi_{N}^{*}\left(\mathbb{I}_{M} \otimes \Delta\right)=$ $\left(\Phi \otimes \mathbb{I}_{N}\right)\left(\Psi \otimes \mathbb{I}_{N}\right)$ as required. Furthermore, $\mathbb{I}_{M}^{\infty} \otimes \mathbb{I}_{N}=\left(\mathbb{I}_{M, N}^{\infty}\right)^{*}\left(\mathbb{I}_{M} \otimes \Delta\right)$. Since $\left(\mathbb{I}_{M, N}^{\infty}\right)_{k}^{*}$ will be non-zero only for $k=1$, we see that the only non-zero term is $\left.\left(\mathbb{I}_{M}^{\infty}\right)_{1}^{*} \otimes\left|\mathbb{I}_{N}\right|^{0}\right)\left(\mathbb{I}_{M} \otimes \Delta_{0}\right)=\left(\mathbb{I}_{M} \otimes \mathbb{I}_{N}\right)\left(\mathbb{I}_{M} \otimes \mathbb{I}_{N}\right)=\mathbb{I}_{M \otimes_{R} N}$. Our map preserves the identity morphisms.

Finally, we verify that the functor preserves homotopy relations. Suppose $\Phi_{N}-\Psi_{N}=$ $D_{M^{\prime}, N} H_{N}+H_{N} D_{M, N}$ for some homotopy map: an order -1 map in $\mathcal{C}\left(M, M^{\prime}\right)$. Since $D_{M^{\prime}, N} H_{N}+H_{N} D_{M, N}=\left(m_{N}^{\prime}+\left(\mathbb{I}_{M^{\prime}} \otimes D_{\mu, N}\right) H_{N}+H_{N}\left(m_{N}+\left(\mathbb{I}_{M} \otimes D_{\mu, N}\right)\right.\right.$, the only terms with image in $M \otimes N$ will be those without a $D_{\mu, N}$ term. Thus, $\Phi_{N}^{*}-\Psi_{N}^{*}=\left(m^{\prime}\right)_{N}^{*} H_{N}+H_{N}^{*} m_{N}$.

Now, let $\mathbb{H}=H_{N}^{*}\left(\mathbb{I}_{M} \otimes \Delta\right)$. Then, using Lemma A.44 above,

$$
\begin{aligned}
\partial^{\bigotimes} \mathbb{H}+ & \mathbb{H} \partial^{\bigotimes} \\
& \left.=\left(m^{\prime}\right)_{N}^{*}\right)\left(\mathbb{I}_{M} \otimes \Delta\right) H_{N}^{*}\left(\mathbb{I}_{M} \otimes \Delta\right)+H_{N}^{*}\left(\mathbb{I}_{M} \otimes \Delta\right)\left(m_{N}^{*}\right)\left(\mathbb{I}_{M} \otimes \Delta\right) \\
& =\left(\left(m^{\prime}\right)_{N}^{*}\right) H_{N}\left(\mathbb{I}_{M} \otimes \Delta\right)+H_{N}^{*} m_{N}\left(\mathbb{I}_{M} \otimes \Delta\right) \\
& =\left(\Phi_{N}^{*}-\Psi_{N}^{*}\right)\left(\mathbb{I}_{M} \otimes \Delta\right) \\
& =\left(\Phi \otimes \mathbb{I}_{N}\right)-\left(\Psi \otimes \mathbb{I}_{N}\right) .
\end{aligned}
$$

Consequently, homotopy equivalent INF-modules will result in chain equivalences of the chain complexes.

Proposition A.45 For each right INF-module $\left(M, D_{M}\right)$ over $\left(A, D_{\mu}\right)$ there is a functor $\mathcal{G}_{\left(M, D_{M}\right)}$ from the category $\mathcal{D}$ of homotopy classes of type $D$ structures over $\left(A, D_{\mu}\right)$ to the homotopy category of chain complexes. $\mathcal{G}_{\left(M, D_{M}\right)}$ is defined by

$$
\begin{aligned}
\mathcal{G}_{\left(M, D_{M}\right)}(N, \delta) & =\left(M \otimes N, \partial^{\bigotimes}\right), \\
\mathcal{G}_{\left(M, D_{M}\right)}(\psi) & =\left[m_{N}^{*}\left(\mathbb{I}_{M} \otimes \bar{\Delta}^{\prime}\right)\left(\mathbb{I}_{M} \otimes \bar{\psi}\right)\left(\mathbb{I}_{M} \otimes \Delta\right)\right],
\end{aligned}
$$

where $\psi$ represents a homotopy class of morphisms of type $D$ structure over $\left(A, D_{\mu}\right)$, and the image $\mathcal{G}_{\left(M, D_{M}\right)}(\psi)$ is the homotopy class of the chain map inside the brackets. We will denote $\mathcal{G}_{\left(M, D_{M}\right)}(\psi)$ by $\mathbb{I}_{M} \otimes \psi$.

Corollary A.46 When $\left(A, D_{\mu}\right)$ has $\mu_{i}=0$ for $i \geq 2$ then the functor $\mathcal{G}_{\left(M, D_{M}\right)}$ can be extended to a functor on the category whose objects are type $D$ structures over $\left(A, D_{\mu}\right)$ and whose morphisms are all the type $D$ morphisms between two type $D$ structures. Homotopic morphisms will be taken by $\mathcal{G}_{\left(M, D_{M}\right)}$ to chain homotopy equivalent chain maps. 
Proof Let $\psi: N \rightarrow A \otimes N^{\prime}$ be an order -1 morphism of type $D$ structures. We define $\mathbb{I}_{M} \otimes \psi: M \otimes N \rightarrow M \otimes N^{\prime}$ to be $\Omega_{1}(\psi)$, or

$$
\mathbb{I}_{M} \otimes \psi=m_{N}^{*}\left(\mathbb{I}_{M} \otimes \bar{\Delta}^{\prime}\right)\left(\mathbb{I}_{M} \otimes \bar{\psi}\right)\left(\mathbb{I}_{M} \otimes \Delta\right) .
$$

By taking $n=1$ in the pairing relation we obtain

$$
(-1)^{1} \Omega_{1}(\psi) \Omega_{0}+(-1)^{0} \Omega_{0} \Omega_{1}(\psi)=\Omega_{1}\left(M_{1}(\psi)\right),
$$

so if $\psi$ is a type $D$ morphism, we obtain $\left(\mathbb{I}_{M} \otimes \psi\right) \partial^{\bigotimes}=\partial^{\bigotimes}\left(\mathbb{I}_{M} \otimes \psi\right)$. Thus, $\left(\mathbb{I}_{M} \otimes \psi\right)$ is a chain map.

Suppose that $H$ is homotopy of type $D$ morphisms $\psi$ and $\phi: \psi-\phi=M_{1}(H)$. If we apply the same identity to $H$ we obtain

$$
(-1)^{2} \Omega_{1}(H) \Omega_{0}+(-1)^{0} \Omega_{0} \Omega_{1}(H)=\Omega_{1}\left(M_{1}(H)\right),
$$

or

$$
\Omega_{1}(H) \partial^{\bigotimes}+\partial^{\bigotimes} \Omega_{1}(H)=\Omega_{1}(\psi-\phi) .
$$

If we let

$$
\mathbb{I}_{M} \otimes H=\Omega_{1}(H)=m_{N}^{*}\left(\mathbb{I}_{M} \otimes \bar{\Delta}^{\prime}\right)\left(\mathbb{I}_{M} \otimes \bar{H}\right)\left(\mathbb{I}_{M} \otimes \Delta\right),
$$

then

$$
\left(\mathbb{I}_{N} \otimes \psi\right)-\left(\mathbb{I}_{N} \otimes \phi\right)=\left(\mathbb{I}_{M} \otimes H\right) \partial^{\bigotimes}+\partial^{\bigotimes}\left(\mathbb{I}_{M} \otimes H\right),
$$

so homotopic type $D$ morphisms will be taken to chain homotopic chain maps. Thus the functor takes morphisms in the homotopy category of type $D$ morphisms to morphisms in the homotopy category of chain complexes.

We have seen that the map $\psi \mapsto \mathbb{I}_{M} \otimes \psi$ takes homotopy classes to homotopy classes. We now verify that it maps the identity correctly, and preserves compositions. The image of $\mathbb{I}_{(N, \delta)}$ is the map $m_{N}^{*}\left(\mathbb{I}_{M} \otimes \bar{\Delta}^{\prime}\right)\left(\mathbb{I}_{M} \otimes \overline{\mathbb{I}_{(N, \delta)}}\right)\left(\mathbb{I}_{M} \otimes \Delta\right)$. This introduces a $1_{A}$ into each term. Since $M$ is strictly unital, the only term remaining will be that employing $m_{2}$. We are thus able to add only one $A$-factor, so $m_{N, 2}^{*}\left(\mathbb{I}_{M} \otimes \mathbb{I}_{(N, \delta)}\right)=$ $m_{N, 2}^{*}\left(\mathbb{I}_{M} \otimes 1_{A} \otimes \mathbb{I}_{N}\right)$ or $\mathbb{I}_{M} \otimes \mathbb{I}_{N}$.

To verify that the functor preserves compositions in the homotopy categories, recall that the composition of two type $D$ morphisms $\psi$ and $\phi$ is given by

$$
M_{2}(\phi, \psi)=\mu_{N^{\prime \prime}}^{*} \bar{\Delta}^{\prime \prime} \bar{\phi} \bar{\Delta}^{\prime} \bar{\psi} \Delta .
$$

As a consequence, we may use the pairing relations, when $\phi$ and $\psi$ are type $D$ morphisms and $n=2$, to get

$$
\begin{aligned}
& (-1)^{0} \partial^{\square} \Omega_{2}(\phi, \psi)+(-1)^{1} \Omega_{1}(\phi) \Omega_{1}(\psi)+(-1)^{2} \Omega_{2}(\phi, \psi) \partial^{\bigotimes} \\
& \quad=(-1)^{0} \Omega_{1}\left(M_{2}(\phi, \psi)\right)+(-1)^{0} \Omega_{2}\left(M_{1}(\phi), \psi\right)+(-1)^{1} \Omega_{2}\left(\phi, M_{1}(\psi)\right) .
\end{aligned}
$$


Since $M_{1}(\phi)=M_{1}(\psi)=0$, this identity becomes

$$
\partial^{\bigotimes} \Omega_{2}(\phi, \psi)+\Omega_{2}(\phi, \psi) \partial^{\bigotimes}=\Omega_{1}(\phi) \Omega_{1}(\psi)+\Omega_{1}\left(M_{2}(\phi, \psi)\right) .
$$

Thus the map $\Omega_{1}(\phi) \Omega_{1}(\psi)$ is chain homotopic to $\Omega_{1}\left(-M_{2}(\phi, \psi)\right)$. Consequently, after modding out by homotopies,

$$
(\mathbb{I} \otimes \phi)(\mathbb{I} \otimes \psi) \simeq(\mathbb{I} \otimes(\phi * \psi)),
$$

and we have verified that the map preserves compositions and thus is a functor.

\section{References}

[1] M M Asaeda, J H Przytycki, A S Sikora, Categorification of the Kauffman bracket skein module of I-bundles over surfaces, Algebr. Geom. Topol. 4 (2004) 1177-1210 MR2113902

[2] M M Asaeda, J H Przytycki, A S Sikora, Categorification of the skein module of tangles, from "Primes and knots" (T Kohno, M Morishita, editors), Contemp. Math. 416, Amer. Math. Soc. (2006) 1-8 MR2276132

[3] D Bar-Natan, On Khovanov's categorification of the Jones polynomial, Algebr. Geom. Topol. 2 (2002) 337-370 MR1917056

[4] J Green, D Bar-Natan, JavaKh (2005) Available at http://katlas.org/wiki/ Khovanov_Homology

[5] B Keller, Introduction to A-infinity algebras and modules, Homology Homotopy Appl. 3 (2001) 1-35 MR1854636

[6] M Khovanov, A categorification of the Jones polynomial, Duke Math. J. 101 (2000) 359-426 MR1740682

[7] M Khovanov, A functor-valued invariant of tangles, Algebr. Geom. Topol. 2 (2002) 665-741 MR1928174

[8] R Lipshitz, P Ozsvath, D Thurston, Bordered Heegaard Floer homology: Invariance and pairing, preprint (2008) arXiv:0810.0687

[9] LP Roberts, A type D structure in Khovanov homology, Adv. Math. 293 (2016) 81-145 MR3474320

Department of Mathematics, University of Alabama Box 870350, Tuscaloosa, AL 35487-0350, United States Iproberts@ua.edu

Received: 1 April 2016 
